\title{
Expansão do sistema FreeFlow-3D para escoamentos com influência da temperatura
}

\author{
Marcelo Henrique Sabatini
}

\author{
Orientador:
}

\section{Prof. Dr. Antonio Castelo Filho}

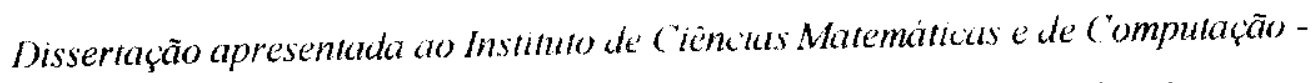
ICMC-USP. como parte dos requasitos pura ohtençióo do titulo de Mestre em

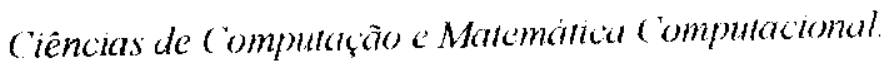

\author{
USP - São Carlos \\ Janeiro/2003
}

\section{"VERSÃO REVISADA APÓS A DEFESA"}

Dala da Delesa:

$12 / 12 / 2002$

Visto do Orientador:

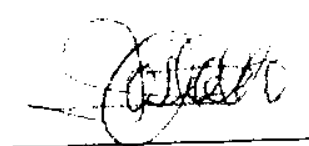


Derlico este trabalho a minha mãe,

Roseli. 


\section{Agradecimentos}

Ao professor Antonio Castclo Filho pela orientação na fase final desse trabalho.

Aos professores do grupo de análise numórica e em especial aos professores Norberto Mangiavacchi e Antonio Castelo Filho, os quais tivexão participaçăo na realizaçăo desso trabalho com valiosas sugestoess, ajuda e boa vontade.

Aos amigos do LCAD, Luciane, Ricardo, Maria Luísa, Juliana, P'io, Helton, Valdemir, Fabrício, Fermando e Hévilla pelas dicas e pelos momentos agradáveis no laboratório.

A todos meus amigos e amigas da USP em especial ao Luis Fernando, Luciano, Thiago, Ricardo, Silvio, Wellington polos bons morrentos de descontração.

A minha mãe pelo apoio e incentivo.

Ao CNPq, pelo suporte financeiro para o desenvolvimento desse projeto.

Finalmerte, agradeço a todas as pessoas que diretamente ou indiretamente colaboraram para a realização desse trabalho. 


\section{Resumo}

O objetivo desse trabalho é extender o ambiente de simulação Freoflow-3D para escoamentos incompressíveis com superfície livre não-isotćrmicos. As ecpuacões de Nivier-Stokes com as condiçôes de fronteira associadas são discretizadas usando o mótodo de diferenças finitas sobre malhas deslocadas. Os efeitos da temperatura são incluidos no código usando a aproximação de Boussinesq e a viscosidade é calculada como uma função da temperatura ou é colocada constante dependendo da escolha do usuário do FreeFlow-3D. Resultados numéricos mostrando a aplicabilidade do ambiente FrecFlow-3D para escoamentos nàoisotérmicos são apresentados e discutidos.

Palavras-Chave: Escoamentos com Superfície: Livre; Fscoamentos Vão-Isotérmicos; Equações de Navier-Stokes; Aproximação de Boussinesq 


\begin{abstract}
The aim of this work is to extend the FreeFlow-31) simmlation system to incompressible? non-isothermal free surface flows. The Navier-Stokes equations together with appropriate boundary conditions are discretized using the finite difference mothod on a staggesed grid. Temperature effects are included in the code by using the Boussinesq approximation and the viscosity is calculated as a function of the temperature or it is set constant depending on the choice of the FreeFlow-3D's user. Numerical results demonstrating the capalsilitics of the FreeFlow-3D code for solving non-isothermal free surface flows are presented and discussed.
\end{abstract}

Keywords: Free Surface Flows; Non-Isothermal Flows; Navicr-Stokes Equations; Boussinesq Approximation 


\section{Sumário}

Introdução

1 Escoamentos Não-Isotérmicos 3

1.1 Conceitos Fundamentais . . . . . . . . . . . . . . . . . . 3

1.2 Fquaçoes de Navier-Stokes . . . . . . . . . . . . . . . . . . . . . 5

1.3 Modelos para Escoamentos Não-Isotérmicos . . . . . . . . . . . . . . 10

1.3.1 Modelo de Boussinesq . . . . . . . . . . . . . . . . . 10

1.3.2 O Cálculo da Viscosidade em Função da Tenuseratura . . . . . . . 11

1.4 Condicoes de Contorno . . . . . . . . . . . . . . . . . 12

1.4.1 Condições de Contorno na Superficie Livre . . . . . . . . . . . . 12

1.4.2 Condiçōes no Contorno Rígido . . . . . . . . . . . . . . . . . . 14

1.5 Adimensionalização das equações . . . . . . . . . . . . . . . . 14

2 Método Computacional $\quad 17$

2.1 Método para o Cálculo da Velocidade, Pressião e Temperatura . . . . . . . 17

2.2 Modificaçoes no Mótodo GENSMAC-3D . . . . . . . . . . . . . . 22

3 Discretizações 23

3.1 Dofiniçäo das Cólulas . . . . . . . . . . . . . . . . . . . . 23

3.2 Discretização do Domínio f.................. 24

3.3 Discretização das Equações de Conservação de Quantidade de Movimento . 25

3.4 Discretizaçäo da Equağão da Energia . . . . . . . . . . . . . 27

3.5 Aproximação dos Termos Convectivos. . . . . . . . . . . . . 28

3.6 Discretização da Equaç̃o de Poisson para

3.7 Condições de Contorno . . . . . . . . . . . . . . . . . . . 34 
3.7.1 Condiçón de Contorno na Superfície Livre . . . . . . . . . . . . . 34

3.7 .2 Condiçōes no Contorno Rígido . . . . . . . . . . . . . 40

3.8 Controle do Passo no Tempo . . . . . . . . . . . . . . . . 45

4 O Ambiente de Simulação FreeFlow-3D para Escoamentos Não-Isotérmicos 46

4.1 O Ambiente de Simulação Freek low-3D . . . . . . . . . . . . . 46

4.2 Estrutura de Dados . . . . . . . . . . . . . . . . . . . 4t

4.3 O Ambiente de Simulaça FrenFlow-3D para Escommentos Não-Isotémincos 49

4.3 .1 O Modelador . . . . . . . . . . . . . . . . . . . 49

4.3 .2 o Simulador. . . . . . . . . . . . . . . . . . . 50

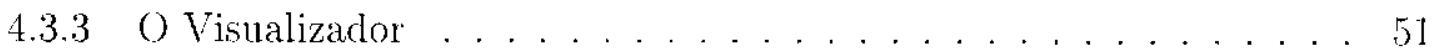

5 Resultados Numéricos

5.1 Convecgão Natural no Cubo Fechado . . . . . . . . . . . . . . . 5.3

5.2 Outros Exemplos Numéricos . . . . . . . . . . . . . . . . . 59

$\begin{array}{llr}6 & \text { Conclusão } & 67\end{array}$

Referências Bibliográficas $\quad 69$ 


\section{Lista de Figuras}

3.1 Tipo de células. . . . . . . . . . . . . . . . . . 24

3.2 Célula computacional, mostrando onde as variáveis são discretizadas. . . . 25

3.3 Estêncil usado para calcular $\phi_{A}$ e $\phi_{B}$ usando vários esquemas. . . . . . . . 29

3.4 Cólula $(\mathrm{S})$ com a face $\left(k+\frac{1}{2}\right)$ adjacente a cólula (L) . . . . . . . . 35

3.5 Exemplo de uma superfície planar inclinada a $45^{\circ} \ldots \ldots$. . . . . . . . . 35

3.6 Célula $(\mathrm{S})$ com as faces $\left(k+\frac{1}{2}\right)$ o $\left(i+\frac{1}{2}\right)$ adjacentes a células $(\mathrm{E}) . \ldots 36$

3.7 Exemplo de uma superfície planar inclinada a $60^{\circ} \ldots \ldots \ldots$. . . . . . . . 36

3.8 Célula (S) com as faces $\left(i+\frac{1}{2}\right),\left(k+\frac{1}{2}\right)$ e $\left(j+\frac{1}{2}\right)$ adjacentes a cólulats (L). . 37

3.9 Célula (S) tendo uma face adjacente a uma célula $(\mathrm{E})$. . . . . . . . . . 38

3.10 Célula (S) tendo duas faces adjacentes a células (E) . . . . . . . . . . 39

3.11 Célula (B) com a face $\left(i+\frac{1}{2}\right)$ adjacente com uma cólula (S) ou (F). . . . 40

3.12 Célula (B) com as faces $\left(i+\frac{1}{2}\right)$ e $\left(j+\frac{1}{2}\right)$ adjacentes a cólulas (S) ou (F). . 41

3.13 Célula (B) com as faces $\left(i+\frac{1}{2}\right),\left(j+\frac{1}{2}\right)$ e $\left(k+\frac{1}{2}\right)$ adjacentes a células (S)

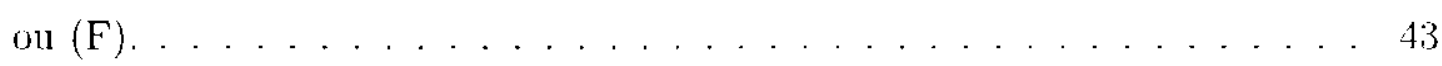

3.14 Célula (B) com a face $\left(i+\frac{1}{2}\right)$ adjacente a cólula (S) ou (F) . . . . . . . 44

4.1 Níveis hierárquicos da estrutura de dados "half-edge" . . . . . . . . . 48

4.2 Entrada de dados. . . . . . . . . . . . . . . . . . . . . . 49

4.3 Entrada dados para simulação do escoamentos não-isotérmicos. . . . . . . 50

4.4 Interface do módulo Modflow-3D para escoamentos näo-isotérnicos, com o modelo para a simulação de uma injeção de um líquido e a janela de entrada dados do objeto do tipo "open box". . . . . . . . . . . 51

4.5 Visualização de um escoamento não-isotérmico: "rendering" tridimensional com recurso de ihuminaçăo (escuerda) e visualização da temporalura (dircita). 52 
4.6 Alguns recursos de visualização do módulo Visflow-31). . . . . . . . . . . 52

5.1 Modelo do problema da convecçào natural. . . . . . . . . . . . . . 54

5.2 Visualizaçäo da temperatura. (a) $R a=10^{3}$; (b) $R a=10^{4}$; (c) $R a=10^{5} \ldots$. 55

5.3 Linhas de temperatura. (a) $R a=10^{3} ;(b) \operatorname{Ra}=10^{4}$; (c) Ra $=10^{5} \ldots \ldots . \quad 55$

5.4 Curvas de níveis da velocidade $u$. (a) $R a=10^{3}$; (b) $R a=10^{4}$; (c) $R a=10^{5}$. 56

5.5 Curvas de níveis da velocidade $z$. (a) $R a=10^{3}$; (b) $R a=10^{4}$; (c) Ra $=10^{5} .56$

5.6 Ajuste de um conjunto de dados pelo método dos mínimos quarlrados utilizando-se o modelo (1.18) para o cálculo da viscosidade. . . . . . . . 56

5.7 Linhas de temperatura, Ra $=10^{4}$. (a) Modelo de Boussinesc com a viscosidade constante; (b) Modelo de Boussinesq com a viscosidade variando em funçào da

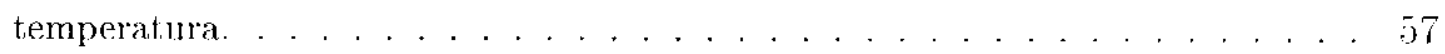

5.8 Normas $\|\cdot\|_{\infty}$ dos erros das componentes de velocidade para cada malha, cm escala logarítimica. . . . . . . . . . . . . . . . . . . . . . . . . .

5.9 Visualizaçäo da temperatura em um recipiente com razão $2: 1: 1$. . . . . 60

5.10 Visualização do modelo. . . . . . . . . . . . . . . . . 61

5.11 Visualizaça tridimensional da lemperatura. . . . . . . . . . . . . . 62

5.12 Visualizagrano bidimensional da temperatura no plano $x \approx$ referente a figural

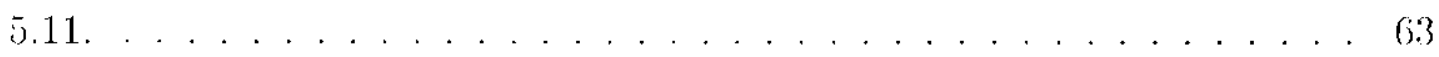

5.13 Representação do perfil de temperatura imposto no fundo do recipiente. . . . . 64

5.14 Representaçấo do fundo do recipiente mostrando $x_{i}, y_{i}, i=0,1,2 \ldots \ldots 4$

5.15 Visualização da temperatura. . . . . . . . . . . . . . . . . . 64

5.16 Visualizaçăo da temperatura, $l=0.75 \mathrm{~s}$, por vários ângulos. . . . . 65

5.17 Ajuste de um conjunto de dados pelo método dos mínimos quadrados utilizando-se o modelo (1.19) para o cálculo da viscosidade. . . . . . . . 65

5.18 Visualização da temperatura. . . . . . . . . . . . . . . . 66

5.19 Visualizaçăo da viscosidade . . . . . . . . . . . . . . . . . 66 


\section{Introdução}

Com o contínuo avanço tecnológico computacional e das técnicas numéricas, é hoje possível a modelagom o simulação de problemas de grande porte em mecanica de fluidos. Nas últimas décadas muitos problemas em nnecânican dos fluídos têm sido estudados sendo que uma classe de problemas que tem tido destaque é o escoamento de fluidos com superfícies livres, o qual possui aplicaçoes industriais tais como o preenchimento de cavidades e injeção de moldes nas indústrias alimentícias, siderúrgicas e de plásticos.

O tratamento de escoamentos com superfícies livres têm obtido sucesso usando o método de difereças finitas. Fin 1965, Harlow e Welch introduziram o método MAC ("Marker-and-Celp") (Harlow o Welch, 1965), que foi uma das primeiras técnicas para a solução de problemas com superfícies livres a apresentar resultados satisfatórios. Nesse método o fluido é representado por partículas virtuais. Fm seguida, 1970, Harlow e Amsden propuseram o método SMAC ("Simplified $M A C$ ") (Amsden e Harlow, 1970) o qual divide o ciclo de cálculo em velocidade e pressão. Assim, algumas das dificuldades encontradas no método MAC, relativas ao processo iterativo no cálculo da velocidade e pressão, deixaram de existir. En 1994, com base no método SMAC, Tomé e McKee desenvolveram o método CENSMAC (Tomé e Mckee, 1994; Tomé et al., 1996b) para cscoannentos bidimensionais de fluidos newtonianos incompressiveis com superfícies livres em domínios complexos.

Existe un projeto em desenvolvimento no ICMC-CSP denominado SNENS: Soluç̃o Numérica das Equaçõos de Navier Stokes, que trata de simulagão de cscommentos de fluidos com superficies livres. O ambiente integrado para modolagem, simulação o visualizaçào do escoamentos incompressiveis com superfícies livres tridimensionais desenvolvido 11 (ssice projeto ó denominado FreeFlow-3D (Castelo Filho et al., 1999). Neste sistema está inplementado o método GENSMAC-3D (Tomé et al., 2001), que é uma extensão do método 


\section{GENSMAC.}

Um dos objetivos desse projeto ó expandir a aplicabilidade do sistema FreeFlow-3D para novos tipos de escoamentos. Assim, essad dissertaça de mestrarlo tem como objetivo desenvolver módulos para o ambiente FreeFlow-3D para modelar, simular e visualizar escoamentos não-isotérmicos, pois é de freqünente interesse a determinação da variação de temperatura no fluido e ou a transfêrencia de calor no fluido.

Os efeitos da temperatura no fluido são representados pela aproximaça de Boussinesq, a qual ó amplamente utilizada nat literatura, e a viscosidade é calculada cm função da temperatura ou é constante dependendo da escolha do usuário. Resultados Numóricos são apresentados.

A disscrtação de mestrado foi dividida nos seguintes capítulos:

- capítulo 1: são apresentados alguns conceitos fundamentais da mecànica dos fluidos, as equaçóes governantes para escoamentos näo-isotérmicos com superfícies livres e a adimensionalização dessàs equagoones;

- capítulo 2: apresenta-se um estudo do método computacional utilizado;

- capítulo 3: as aproximações por diferenças finitas das equaçooes introduzidas no capítulo 1 são apresentadas, assim como técnicas para o tratamento das condiçoes de contorno na superfície livre e no contorno rígido:

- capítulo 4: apresenta-se mana descriçäo do sistema Freeflow-3D a as modificaçoes feitias em seus mórlulos;

- capítulo 5: os resultados numéricos obtidos são apresentados;

- capítulo 6: são apresentadas as conclusões sobre esse trabalho. 


\section{Capítulo 1}

\section{Escoamentos Não-Isotérmicos}

Para se poder simular um escoamento em que o domínio e/ou fluido năo estão na mesma temperatura, as equaçōes de Navier-Stokes devem ser ajustadas corretamente. P'ortanto, este capítulo tem por objetivo apresentar de forma resumida os conceitos básicos da mecânica dos fluidos (Fox o McDonald. 1995; Schmidt et al., 1996), as equarcoes de NivierStokes para simular escoamentos incompressíveis com superfície livre näo-isotérmicos, definir as condiçoes de contorno a a adimensionalizaçầ destas equaçôes.

\subsection{Conceitos Fundamentais}

Fluido é uma substância que se deforma continuamente sob a aplicação de uma tensão de cisalhamento $\tau$, não importando o quão pequena possa ser essa lensão, isto é, cle escoa. () campo de escoamento é uma representação desse movimento no espaço en dilerentes instantes. A propriedade que descreve o campo do escoamento a o votor velocidarle u.

(Os fluidos podem ser classificados como:

- newtonianos: fluidos nos quais existe uma relaçäo linear entre a tensão de cisalhamento aplicada e a taxa da deformação resultante:

- nấo-newtonianos: fluidos que não são newtonianos.

Gases e líquidos sẫo, en geral, fluidos newtonianos como por exemplo: ar e águri. O sarıue, porém é $1 \mathrm{~mm}$ fluido nä̀-newtoniano. 
A propriedade que relaciona a tensão de cisalhamento com a taxa de deformadsão associada ao movimento do fluido é a viscosidade $\mu$. A lei de Newton da viscosidade estabelece que, para uma dada taxa de deformaģio angular de um fluido, a tensăo de cisallhamento é diretamente proporcional à viscosidade. A viscosidade fé chamada de viscosidade absoluta ou dinâmica para se cvitar confusẫo com a viscosidade cinemática relação entre a viscosidade e a massa específica, isto $6, \nu=\frac{\mu}{\rho}$. Vel e oléo lubrificante são exemplos de líquidos muito viscosos, entretanto a água o o ar têm baixas viscosidades.

Um escoamento é considerado incompressível se a variação da massa específica do fluido for desprezivel. Caso contrário, o escoamento é considerado compressível. Un escoamento é classificado como estacionário se as grandezas como velocidade e pressão naio variam com o tempo; caso contrário o escoamento é denominado transiente. () escommento é considerado interno quando o fluido está totalmente cercado por paredess é chassificado como externo quando o fluido não está confinado por paredes e pode on não apresentar superfícies livres. Escoamento laminar é aquele no qual as camadlas muitos finas (laminas) de fluido parecen deslizar umas sobre as outras. Escoamento turbulento é acfucle enn que ocorre movimentos desordenados de pequenas massas de fluido, contrariando o cue ocorre no escoamento laminar.

Neste trabalho, as equações governantes e condiçōes de contorno serão resolvidas na forma adimensional. Algumas constantes de adimensionalização importantes por caracterizarem o escoamento são definidas a seguir:

- número de Reynolds: $\mathrm{Re}=\frac{\rho L L}{\mu}=\frac{V L}{\nu}$, onde $L$ é um comprimento característico, $U$ uma velocidade característica e $\nu$ a viscosidade cinemática. () número de Revnolds é a razão entre as forças inerciais e as forças viscossas de um fluido e é um critério que pode indicar se o escoamento é laminar ou turbulento:

- número de Froude: $\mathrm{Fr}=\frac{U}{\sqrt{L g}}$, onde g é a constante gravitacional. O múmero de Froude é a razão entre forças de inércia e de gravidade e tom papel importante em escoamentos com superfícies livres;

- número de Pranditl: $\operatorname{Pr}=\frac{\nu}{a}$ en que $\alpha=\frac{k}{\rho c_{i}}$ é a difusividade térmica do fluido. O número de Prandtl é a razão entre a viscosidade cincmática e a difusividade térmica do fluido e aparece em escoamentos não-isotérmicos: 
- número de Rayleagh: Ra $=\frac{g \beta \Delta T h^{3}}{\nu^{2}} \operatorname{Pr}$ em que $\beta$ ó o coeficionte de expansäo témica do fluido e $\Delta T$ a diferença entre as temperaturas máxima e mínima. () número de Rayleigh é a razão entre as forças de cmpuxo e as forças de difusão e aparece em escoamentos não-isotérmicos;

- Número de Nusselt: $\mathrm{Nu}=\frac{h L}{k}$ em que hé o coeficiente de trasferencia de calor o $k$ a condutividade térmica do fluido. O número de Nusselt é a razào entre a transferencia de calor e a condutividade térmica do fluido e aparece em escoamentos não-isotérmicos.

\subsection{Equações de Navier-Stokes}

As equaçōes que modelam os escoamentos de fluidos são as de Navier-Stokes. Essas equações representam os príncipios básicos das leis de conservação (lortuna, 2000).

- conservação de massa:

$$
\nabla \cdot \mathbf{u}=0
$$

- conservação de quantidarle de movimento:

$$
\rho \frac{D \mathbf{u}}{D t}=\nabla \cdot \sigma+\rho \mathrm{g}
$$

em que $\rho$ ć a massa especílica, $\mathbf{u}(\mathbf{x}, t)$ o vetor velocidade, $\sigma$ o tensor de tensóes e $\mathrm{g}$ o campo gravitacional do fluido. Como pretendemos tratiar de escoamentos de $u m$ fluido generalizado, consideram-se as seguintes equaçoes constitutivas

$$
\begin{gathered}
\sigma=-p \mathbf{I}+\tau \\
\tau=2 \mu(T) \mathbf{d} \\
\mathbf{d}=\frac{1}{2}\left[(\nabla \mathbf{u})+(\nabla \mathbf{u})^{\prime}\right]
\end{gathered}
$$


em que $p=p(\mathbf{x}, t)$ ć a pressão, I o tensor identidade, $\tau$ o tensol extra tensão, $\mathbf{d}$ o tensor razão de deformaçăo de um fluido e $\mu(T)$ re uma expressão gue descreve o comportamento da viscosidade en função da temperatura.

Em coordenadas cartesianas tridimensionais tem-se

$$
\mathbf{u}(\mathbf{x}, t)=\left(\begin{array}{c}
u(\mathbf{x}, t) \\
v(\mathbf{x}, t) \\
w(\mathbf{x}, t)
\end{array}\right), \quad \mathbf{x}=-\left(\begin{array}{l}
x \\
y \\
z
\end{array}\right)
$$

entã̃o

$$
(\nabla \mathbf{u})^{T}=\left(\begin{array}{ccc}
\frac{\partial u}{\partial x} & \frac{\partial u}{\partial y} & \frac{\partial u}{\partial z} \\
\frac{\partial w}{\partial x} & \frac{\partial v}{\partial y} & \frac{\partial w}{\partial z} \\
\frac{\partial w}{\partial x} & \frac{\partial w}{\partial y} & \frac{\partial w}{\partial z}
\end{array}\right), \quad(\nabla \mathbf{u})=\left(\begin{array}{ccc}
\frac{\partial u}{\partial x} & \frac{\partial w}{\partial x} & \frac{\partial w}{\partial x} \\
\frac{\partial u}{\partial y} & \frac{\partial w}{\partial y} & \frac{\partial w}{\partial y} \\
\frac{\partial u}{\partial z} & \frac{\partial w}{\partial z} & \frac{\partial w}{\partial z}
\end{array}\right)
$$

Então (1.5) torma-se

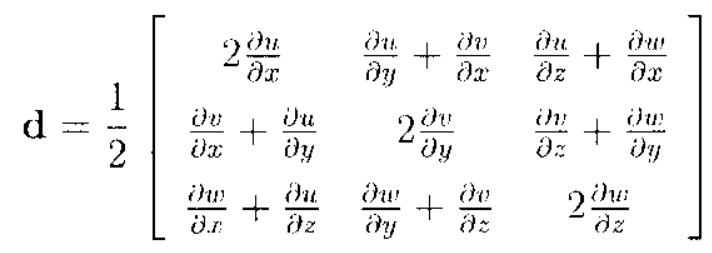

e portanto (1.4) é dada por

$$
\tau=\mu(T)\left[\begin{array}{ccc}
2 \frac{\partial u}{\partial x} & \frac{\partial u}{\partial y}+\frac{\partial w}{\partial x} & \frac{\partial u}{\partial z}+\frac{\partial w}{\partial x} \\
\frac{\partial n}{\partial x}+\frac{\partial u}{\partial y} & 2 \frac{\partial y}{\partial y} & \frac{\partial v}{\partial z}+\frac{\partial w}{\partial y} \\
\frac{\partial w}{\partial x}+\frac{\partial u}{\partial z} & \frac{\partial w}{\partial y}+\frac{\partial v}{\partial z} & 2 \frac{\partial w}{\partial z}
\end{array}\right]
$$

Logo (1.3) pode ser escrita como 


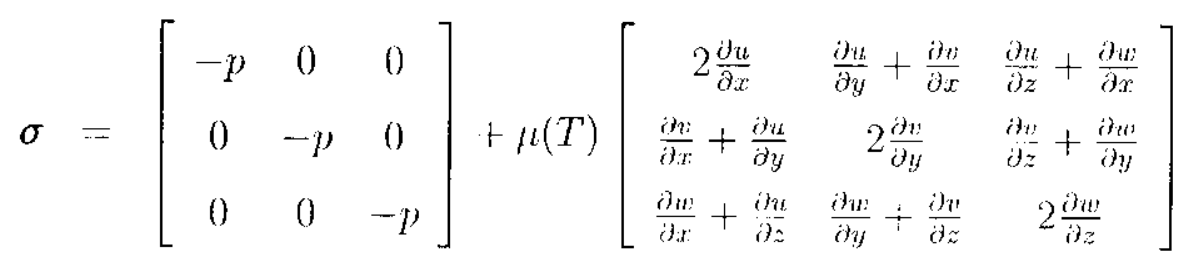

$$
\begin{aligned}
& =\left[\begin{array}{ccc}
p+2 \mu(T) \frac{\partial y_{u}}{\partial x} & \mu(T)\left(\frac{\partial u}{\partial y}+\frac{\partial y_{z}}{\partial x}\right) & \mu(T)\left(\frac{\partial u}{\partial z}+\frac{\partial w}{\partial x}\right) \\
\mu(T)\left(\frac{\partial w}{\partial x}+\frac{\partial u}{\partial y}\right) & -p+2 \mu(T) \frac{\partial \partial_{w}}{\partial y} & \mu(T)\left(\frac{\partial w}{\partial z}+\frac{\partial w}{\partial y}\right) \\
\mu(T)\left(\frac{\partial w}{\partial x}+\frac{\partial u}{\partial z}\right) & \mu(T)\left(\frac{\partial w}{\partial y}+\frac{\partial y}{\partial z}\right) & -p+2 \mu(T) \frac{\partial w}{\partial z}
\end{array}\right] .
\end{aligned}
$$

Assim

$$
\nabla \cdot \sigma=\left[\begin{array}{l}
A_{1} \\
A_{2} \\
A_{3}
\end{array}\right]
$$

em que

$$
\begin{aligned}
A_{1} & =-\frac{\partial p}{\partial x}+\mu(T)\left(\frac{\partial^{2} u}{\partial x^{2}}+\frac{\partial^{2} u}{\partial y^{2}}+\frac{\partial^{2} u}{\partial z^{2}}\right)+\mu(T) \frac{\partial}{\partial x}\left(\frac{\partial u}{\partial x}+\frac{\partial u}{\partial y}+\frac{\partial w}{\partial z}\right) \\
& +2 \frac{\partial \mu(T)}{\partial x} \frac{\partial u}{\partial x}+\frac{\partial \mu(T)}{\partial y}\left(\frac{\partial u}{\partial y}+\frac{\partial v}{\partial x}\right)+\frac{\partial \mu(T)}{\partial z}\left(\frac{\partial u}{\partial z}+\frac{\partial w}{\partial x}\right) \\
A_{2} & =-\frac{\partial p}{\partial y}+\mu(T)\left(\frac{\partial^{2} v}{\partial x^{2}}+\frac{\partial^{2} v}{\partial y^{2}}+\frac{\partial^{2} v}{\partial z^{2}}\right)+\mu(T) \frac{\partial}{\partial y}\left(\frac{\partial u}{\partial x}+\frac{\partial v}{\partial y}+\frac{\partial w}{\partial z}\right) \\
& +2 \frac{\partial \mu(T)}{\partial y} \frac{\partial v}{\partial y}+\frac{\partial \mu(T)}{\partial x}\left(\frac{\partial v}{\partial x}+\frac{\partial u}{\partial y}\right)+\frac{\partial \mu(T)}{\partial z}\left(\frac{\partial v}{\partial z}+\frac{\partial w}{\partial y}\right) \\
A_{3} & =-\frac{\partial p}{\partial z}+\mu(T)\left(\frac{\partial^{2} w}{\partial x^{2}}+\frac{\partial^{2} w}{\partial y^{2}}+\frac{\partial^{2} w}{\partial z^{2}}\right)+\mu(T) \frac{\partial}{\partial z}\left(\frac{\partial u}{\partial x}+\frac{\partial v}{\partial y}+\frac{\partial w}{\partial z}\right) \\
& +2 \frac{\partial \mu(T)}{\partial z} \frac{\partial w}{\partial z}+\frac{\partial \mu(T)}{\partial x}\left(\frac{\partial w}{\partial x}+\frac{\partial u}{\partial z}\right)+\frac{\partial \mu(T)}{\partial y}\left(\frac{\partial w}{\partial y}+\frac{\partial v}{\partial z}\right)
\end{aligned}
$$

Utilizando a equação da continuidade em $\nabla \cdot \sigma$ reduz-se a

$$
\nabla \cdot \sigma=\left[\begin{array}{c}
B_{1} \\
B_{2} \\
B_{3}
\end{array}\right]
$$


cril que

$$
\begin{aligned}
B_{1} & =-\frac{\partial p}{\partial x}+\mu(T)\left(\frac{\partial^{2} u}{\partial x^{2}}+\frac{\partial^{2} u}{\partial y^{2}}+\frac{\partial^{2} u}{\partial z^{2}}\right)+2 \frac{\partial \mu(T)}{\partial x} \frac{\partial u}{\partial x} \\
& +\frac{\partial \mu(T)}{\partial y}\left(\frac{\partial u}{\partial y}+\frac{\partial v}{\partial x}\right)+\frac{\partial \mu(T)}{\partial z}\left(\frac{\partial u}{\partial z}+\frac{\partial w}{\partial x}\right) \\
B_{2} & =-\frac{\partial p}{\partial y}+\mu(T)\left(\frac{\partial^{2} v}{\partial x^{2}}+\frac{\partial^{2} v}{\partial y^{2}}+\frac{\partial^{2} v}{\partial z^{2}}\right)+2 \frac{\partial \mu(T)}{\partial y} \frac{\partial v}{\partial y} \\
& +\frac{\partial \mu(T)}{\partial x}\left(\frac{\partial v}{\partial x}+\frac{\partial u}{\partial y}\right)+\frac{\partial \mu(T)}{\partial z}\left(\frac{\partial v}{\partial z}+\frac{\partial w}{\partial y}\right) \\
B_{3}= & -\frac{\partial p}{\partial z}+\mu(T)\left(\frac{\partial^{2} u}{\partial x^{2}}+\frac{\partial^{2} w}{\partial y^{2}}+\frac{\partial^{2} w}{\partial z^{2}}\right)+2 \frac{\partial \mu(T)}{\partial z} \frac{\partial w}{\partial z} \\
& +\frac{\partial \mu(T)}{\partial x}\left(\frac{\partial w}{\partial x}+\frac{\partial u}{\partial z}\right)+\frac{\partial \mu(T)}{\partial y}\left(\frac{\partial w}{\partial y}+\frac{\partial v}{\partial z}\right) .
\end{aligned}
$$

Logo (1.6) pode ser escrita na forma vetorial

$$
\nabla \cdot \sigma=-\nabla p+\mu(T) \nabla^{2} \mathbf{u}+(\nabla \mu(T))\left[(\nabla \mathbf{u})+(\nabla \mathbf{u})^{T}\right] .
$$

$A_{\text {plicando-se } 0}$ operador diferencial

$$
\frac{D}{D t}=\frac{\partial}{\partial t}+(\mathbf{u} \cdot \nabla)
$$

sobre o vetor $\mathbf{u}$ obtem-se

$$
\frac{D \mathbf{u}}{D t}=\frac{\partial \mathbf{u}}{\partial t}+(\mathbf{u} \cdot \nabla) \mathbf{u}
$$

que na forma conscrvativa ó dado por

$$
\frac{D \mathbf{u}}{D t}=\frac{\partial \mathbf{u}}{\partial t}+\nabla \cdot(\mathbf{u u})
$$

onde

$$
\nabla \cdot(\mathbf{u u})=\nabla \cdot\left[\begin{array}{ccc}
u^{2} & (w v) & (u u:) \\
(v u) & v^{2} & (v w) \\
(w u) & (w v) & w^{2}
\end{array}\right]=\left[\begin{array}{c}
\frac{\partial u^{2}}{\partial x}+\frac{\partial(w w)}{\partial t y}+\frac{\partial(u w)}{\partial z} \\
\frac{\partial(v u)}{\partial x}+\frac{\partial v^{2}}{\partial \eta}+\frac{\partial(v w)}{\partial z} \\
\frac{\partial(w u)}{\partial x}+\frac{\partial(w w)}{\partial y}+\frac{\partial u v^{2}}{\partial z}
\end{array}\right]
$$


$\Lambda$ ssim

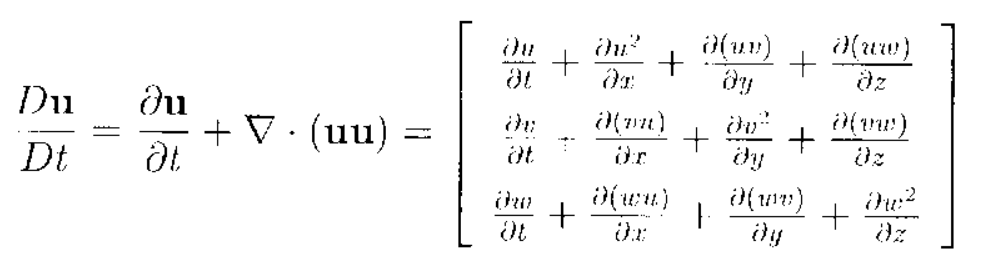

Dividindo (1.2) por $\rho$ tem-se

$$
\frac{D \mathbf{u}}{D t}=\frac{1}{\rho} \nabla \cdot \sigma+\mathbf{g}
$$

Assim a partir de (1.6), (1.7) e (1.8) obtem-se as cpuaçóse de conservagäo dle quantidade de movimento na forma conservativa o cm coordenalas cartesianas tridinensionais

$$
\begin{aligned}
& \frac{\partial u}{\partial t}+\frac{\partial u^{2}}{\partial x}+\frac{\partial(u v)}{\partial y}+\frac{\partial(u w)}{\partial z}=-\frac{1}{\rho} \frac{\partial p}{\partial x}+\nu(T)\left(\frac{\partial^{2} u}{\partial x^{2}}+\frac{\partial^{2} u}{\partial y^{2}}+\frac{\partial^{2} u}{\partial z^{2}}\right) \\
& +\left[2 \frac{\partial u}{\partial x} \frac{\partial \nu(T)}{\partial x}+\left(\frac{\partial u}{\partial y}+\frac{\partial u}{\partial x}\right) \frac{\partial \nu(T)}{\partial y}+\left(\frac{\partial u}{\partial z}+\frac{\partial w}{\partial x}\right) \frac{\partial \nu(T)}{\partial z}\right]+g_{x} \\
& \frac{\partial v}{\partial t}+\frac{\partial(u v)}{\partial x}+\frac{\partial v^{2}}{\partial y}+\frac{\partial(v w)}{\partial z}=-\frac{1}{\rho} \frac{\partial p}{\partial y}+\nu^{\prime}\left(I^{\prime}\right)\left(\frac{\partial^{2} v}{\partial x^{2}}+\frac{\partial^{2} v}{\partial y^{2}}+\frac{\partial^{2} v}{\partial z^{2}}\right) \\
& +\left[\left(\frac{\partial u}{\partial y}+\frac{\partial v}{\partial x}\right) \frac{\partial \nu(T)}{\partial x}+2 \frac{\partial v}{\partial y} \frac{\partial \nu(T)}{\partial y}+\left(\frac{\partial w}{\partial y}+\frac{\partial v}{\partial z}\right) \frac{\partial \nu(T)}{\partial z}\right]+g_{y} \\
& \frac{\partial w}{\partial t}+\frac{\partial(u w)}{\partial x}+\frac{\partial(n w)}{\partial y}+\frac{\partial w^{2}}{\partial z}=-\frac{1}{\rho} \frac{\partial p}{\partial z}+\nu(T)\left(\frac{\partial^{2} w}{\partial x^{2}}+\frac{\partial^{2} w}{\partial y^{2}}+\frac{\partial^{2} w}{\partial z^{2}}\right) \\
& +\left[\left(\frac{\partial u}{\partial z}+\frac{\partial w}{\partial x}\right) \frac{\partial \nu(T)}{\partial x}+\left(\frac{\partial v}{\partial z}+\frac{\partial u}{\partial y}\right) \frac{\partial \nu(T)}{\partial y}+2 \frac{\partial w}{\partial z} \frac{\partial \nu(T)}{\partial z}\right]+y_{z}
\end{aligned}
$$

om que $\mathrm{g}=\left(g_{s}, g_{y}, g_{z}\right)^{T}$

- conservação de energia: na forma conservativa e om coordenadas cartesianas tridimonsionais a equação da energiá ć dada por

$$
\frac{\partial T}{\partial t}+\frac{\partial(u T)}{\partial x}+\frac{\partial(u T)}{\partial y}+\frac{\partial(u T)}{\partial z}=\alpha\left(\frac{\partial^{2} T}{\partial x^{2}}+\frac{\partial^{2} T}{\partial y^{2}}+\frac{\partial^{2} T}{\partial z^{2}}\right)
$$

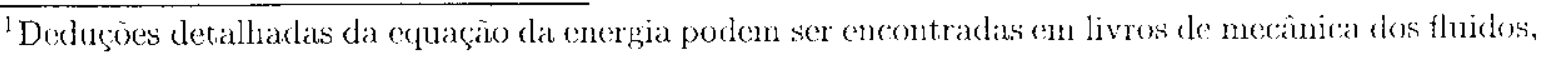
por excmplo (Fletcher, 1988). 
em que $T$ é a temperatura e $\alpha=\frac{\kappa}{\rho c_{p}}$ é o coeficiente de difusividade térmica do fluido; fi é o coeficiente de condutividade térmica do fluido e ep o calor específico a pressáo constante do fluido.

\subsection{Modelos para Escoamentos Não-Isotérmicos}

Mudanças na temperatura do fluido causam mudanças em suas propriedades (Griebel ct al., 1997). Por exemplo, se um fluido é aquecido seu volume aumenta, fazendo com que ele se torne mais leve. Nos últimos anos, modelos para a simulação de escoamentos nãoisotérmicos tem sido cliscutidos onde a grande maioria utiliza a aproximacäo de Bonssinesq. Foram implementados modelos para a simulação do escoamentos näo-isotérmicos utilizando-se a aproximagão de Boussinesq e a viscosidade variando en funçăo da ten-11peratura.

\subsubsection{Modelo de Boussinesq}

A aproximaçăo de Boussinesq (Fortuna, 2000; Griebel et al.. 1997), considera constante? todas as propriedades do fluido exceto a massa específica no termo fonte das equaçocs de conservação de quantidade de movimento, a qual é assumida uma relação linear contre a massa específica $\rho$ e a temperatura $T$, isto é,

$$
\rho(T)=\rho_{\infty}+\left.\frac{\partial \rho}{\partial T}\right|_{T_{\infty}}\left(T-T_{\infty}\right)+O\left(T-T_{\infty}\right)^{2}
$$

que pode ser escrita como

$$
\rho(T) \approx \rho_{\infty}\left[1-\beta\left(T^{\prime}(\mathbf{x}, t)-T_{\infty}\right)\right]
$$

em que $\rho_{\infty}$ e $T_{\infty}$ são valores de refêrencia da massa específica c da tomperatura respectivamente, $\mathrm{e} \beta=-\left.\frac{1}{\rho_{\infty}} \frac{\partial \rho}{\partial T}\right|_{T_{\infty \times},}$ o o cocficiente de expansăo térmica do fluido.

Assim, as equaçôs de conservação de quantidade de movimento (1.9), (1.10) e (1.11) dadas anteriormente podem ser escritas como 


$$
\begin{aligned}
& \frac{\partial u}{\partial t}+\frac{\partial u^{2}}{\partial x}+\frac{\partial(u v)}{\partial y}+\frac{\partial(u w)}{\partial z}=-\frac{1}{\rho} \frac{\partial p}{\partial x}+\nu(T)\left(\frac{\partial^{2} u}{\partial x^{2}}+\frac{\partial^{2} u}{\partial y^{2}}+\frac{\partial^{2} u}{\partial z^{2}}\right) \\
& +\left[2 \frac{\partial u}{\partial x} \frac{\partial \nu(T)}{\partial x}+\left(\frac{\partial u}{\partial y}+\frac{\partial v}{\partial x}\right) \frac{\partial \nu(T)}{\partial y}+\left(\frac{\partial u}{\partial z}+\frac{\partial u}{\partial x}\right) \frac{\partial \nu(T)}{\partial z}\right] \\
& +\left[1-\beta\left(T(\mathbf{x}, t) \cdots T_{\infty}\right)\right] g_{x} \\
& \frac{\partial v}{\partial t}+\frac{\partial(u v)}{\partial x}+\frac{\partial v^{2}}{\partial y}+\frac{\partial(v w)}{\partial z}=-\frac{1}{\rho} \frac{\partial p}{\partial y}+\nu(T)\left(\frac{\partial^{2} v}{\partial x^{2}}+\frac{\partial^{2} v}{\partial y^{2}}+\frac{\partial^{2} v}{\partial z^{2}}\right) \\
& +\left[\left(\frac{\partial u}{\partial y}+\frac{\partial v}{\partial x}\right) \frac{\partial \nu(T)}{\partial x}+2 \frac{\partial v}{\partial y} \frac{\partial \nu(T)}{\partial y}+\left(\frac{\partial u}{\partial y}+\frac{\partial v}{\partial z}\right) \frac{\partial v(T)}{\partial z}\right] \\
& +\left[1-\beta\left(T(\mathbf{x}, \ell)-T_{\infty}\right)\right] g_{y} \\
& \frac{\partial w}{\partial t}+\frac{\partial(u w)}{\partial x}+\frac{\partial(w w)}{\partial y}+\frac{\partial w^{2}}{\partial z}=-\frac{1}{\rho} \frac{\partial p}{\partial z}+\nu(T)\left(\frac{\partial^{2} w}{\partial x^{2}}+\frac{\partial^{2} w}{\partial y^{2}}+\frac{\partial^{2} w}{\partial z^{2}}\right) \\
& +\left[\left(\frac{\partial u}{\partial z}+\frac{\partial w}{\partial x}\right) \frac{\partial u(T)}{\partial x}+\left(\frac{\partial v}{\partial z}+\frac{\partial w}{\partial y}\right) \frac{\partial u(T)}{\partial y}+2 \frac{\partial w}{\partial z} \frac{\partial u(T)}{\partial z}\right] \\
& +\left[1-\beta\left(T(\mathbf{x}, \iota)-T_{\infty}\right)\right] g_{z}
\end{aligned}
$$

em que $\left[1-\beta\left(T(\mathrm{x}, t)-T_{\infty}\right)\right] \mathrm{g}$ é denominado o termo fonte. ${ }^{2}$

Para determinarmos a conveção e a difusão da temperatura no domínio, deve-se resolver a equação da energia (1.12).

Portanto as equaçoes a serem resolvidas cm escoamentos nâo-isotérnicos ntilizandose a aproximação de Boussinesq são as equaçoes $(1.12),(1.15),(1.16),(1.17)$, além da equaçăo da continuidado (1.1).

\subsubsection{O Cálculo da Viscosidade em Função da Temperatura}

A viscosidade $\nu(T)$ é uma funçäo conhecida representiando a dependencia do fluido (mo funçăo da temperatura. Para o tratamento da viscosidade, neste trabalho utilizam-so: dois modelos, os quais são:

$$
\nu(T)=a T^{2}+b T+c
$$

(?

2 Para o modelo de Boussinesa pode se considerar $\rho=\rho_{\infty}$. Ver (Silva, 2002). 


$$
\nu(T)=\frac{1}{a T^{2}+b T+c} .
$$

Nos modelos acima a, b e c são constantes a serem determinadas de forma a ajustar um conjunto de dados conhecidos da viscosidade em funça da temperatura pelo método dos mínimos quadrados, sendo que o modelo que melhor ajustiar os dados deverá ser escolhido. A escolha desses modelos foi feita observando-se conjuntos de dados da viscosidade cm

função da temperatura, de onde notamos que o modelo (1.18) molhor ajusta os dados para fluidos gasosos enquanto que o modelo (1.19) fluidos líquidos. Na literatura pode ser encontrada tabelas de propriedades físicas de fluidos contendo esses dados, como por exerrplo o ar (Griebel et al., 1997).

\subsection{Condições de Contorno}

\subsubsection{Condições de Contorno na Superficie Livre}

Na superfície livre as condições de fronteira são obtidas impondo que tensões normal e tangencial se anulem. Na ausência de tensäo na superfície estas condições porlem ser representadas por (Tomé et al., 1996a)

$$
\begin{gathered}
\mathrm{n} \cdot \sigma \cdot \mathrm{n}=0 \\
\mathrm{~m} 1 \cdot \sigma \cdot \mathbf{n}=0 \\
\mathrm{~m} 2 \cdot \sigma \cdot \mathrm{n}=0
\end{gathered}
$$

em que $\mathbf{n}=\left(n_{x}, n_{y}, n_{z}\right)$ é o vetor normal unitário, $\mathbf{m} \mathbf{1}=\left(m 1_{x}, m 1_{y}, m 1_{z}\right)$ : $\mathbf{m} \mathbf{2}=$ $\left(m 2_{x}, m 2_{y}, m 2_{z}\right)$ são os vetores tangenciais à superfície respectivamente. () tensor de tensões $\sigma$ é dado por (1.3).

Escrevendo (1.20) cm coordenadas cartesianas tridimensionais tem-se 


$$
\begin{aligned}
\mathbf{n} \cdot \sigma \cdot \mathbf{n} & =\left(-p+2 \mu(T) \frac{\partial u}{\partial x}\right) n_{x}^{2}+\mu(T)\left(\frac{\partial u}{\partial y}+\frac{\partial u}{\partial x}\right) n_{x} n_{y}+\mu(T)\left(\frac{\partial u}{\partial z}+\frac{\partial w}{\partial x}\right) n_{x} n_{z} \\
& +\mu(T)\left(\frac{\partial v}{\partial x}+\frac{\partial u}{\partial y}\right) n_{x} n_{y}+\left(-p+2 \mu(T) \frac{\partial v}{\partial y}\right) n_{y}^{2}+\mu(T)\left(\frac{\partial v}{\partial z}+\frac{\partial w}{\partial y}\right) n_{y} n_{z} \\
& +\mu(T)\left(\frac{\partial u}{\partial z}+\frac{\partial w}{\partial x}\right) n_{x} n_{z}+\mu(T)\left(\frac{\partial u}{\partial y}+\frac{\partial v}{\partial z}\right) n_{y} n_{z}+\left(-p+2 \mu(T) \frac{\partial w}{\partial z}\right) n_{z}^{2} \\
& =-p\left(n_{x}^{2}+n_{y}^{2}+n_{z}^{2}\right)+2 \mu(T)\left[\frac{\partial u}{\partial y} n_{x}^{2}+\frac{\partial u}{\partial y} n_{y}^{2}+\frac{\partial w}{\partial z} n_{z}^{2}+\left(\frac{\partial u}{\partial y}+\frac{\partial v}{\partial x}\right) n_{x} n_{y}\right. \\
& \left.+\left(\frac{\partial u}{\partial z}+\frac{\partial w}{\partial x}\right) n_{x} n_{z}+\left(\frac{\partial v}{\partial z}+\frac{\partial w}{\partial y}\right) n_{y} n_{z}\right]
\end{aligned}
$$

c como $\|\mathbf{n}\|=1$ obténl-se

$$
\begin{aligned}
& -p+2 \mu(T)\left[\frac{\partial u}{\partial y} n_{x}^{2}+\frac{\partial v}{\partial y} n_{y}^{2}+\frac{\partial w}{\partial z} n_{z}^{2}+\left(\frac{\partial u}{\partial y}+\frac{\partial v}{\partial x}\right) n_{x} n_{y}\right. \\
& \left.+\left(\frac{\partial u}{\partial z}+\frac{\partial w}{\partial x}\right) n_{x} n_{z}+\left(\frac{\partial v}{\partial z}+\frac{\partial w}{\partial y}\right) n_{y} n_{z}\right]=0 .
\end{aligned}
$$

Do mesmo modo, para (1.21) tem-se

$$
\begin{aligned}
\mathbf{m} \mathbf{1} \cdot \boldsymbol{\sigma} \cdot \mathbf{n} & =-p\left(n_{x} m 1_{x}+n_{y} m 1_{x}+n_{z} m 1_{z}\right)+2 \mu(T) \frac{\partial u}{\partial x} n_{x} m 1_{x}+2 \mu(T) \frac{\partial v}{\partial y} n_{y} m 1_{y} \\
& +2 \mu(T) \frac{\partial w}{\partial z} n_{z} m 1_{z}+\mu(T)\left(\frac{\partial u}{\partial y}+\frac{\partial v}{\partial x}\right)\left(n_{y} m 1_{x}+n_{x} m 1_{y}\right) \\
& +\mu(T)\left(\frac{\partial u}{\partial z}+\frac{\partial w}{\partial x}\right)\left(m_{z} m 1_{x}+n_{x} m 1_{z}\right)+\mu(T)\left(\frac{\partial w}{\partial z}+\frac{\partial w}{\partial y}\right)\left(n_{z} m 1_{y}+n_{y} m 1_{z}\right)
\end{aligned}
$$

c como $\mathrm{n} \cdot \mathrm{m} 1=0$ obtém-se

$$
\begin{aligned}
& 2 \frac{\partial u}{\partial x} n_{x} m 1_{x}+2 \frac{\partial v}{\partial y} n_{y} m 1_{y}+2 \frac{\partial w}{\partial z} n_{z} m 1_{z}+\left(\frac{\partial u}{\partial y}+\frac{\partial v}{\partial x}\right)\left(n n_{y} m 1_{x}+n_{x} m 1_{y}\right) \\
& +\left(\frac{\partial u}{\partial z}+\frac{\partial w}{\partial x}\right)\left(n_{z} m 1_{x}+n_{x} m 1_{z}\right)+\left(\frac{\partial v}{\partial z}+\frac{\partial w}{\partial y}\right)\left(n_{z} m 1_{y}+n_{y} m 1_{z}\right)=0
\end{aligned}
$$

(? analogamente para (1.22) tem-se

$$
\begin{aligned}
& 2 \frac{\partial u}{\partial x} n_{x} m 2_{x}+2 \frac{\partial v}{\partial y} n_{y} m 2_{y}+2 \frac{\partial w}{\partial z} n_{z} m 2_{z}+\left(\frac{\partial u}{\partial y}+\frac{\partial v}{\partial x}\right)\left(n m 2_{x}+n m_{x} m 2_{y}\right) \\
& +\left(\frac{\partial u}{\partial z}+\frac{\partial w}{\partial x}\right)\left(n_{z} m 2_{x}+n_{x} m 2_{z}\right)+\left(\frac{\partial v}{\partial z}+\frac{\partial w}{\partial y}\right)\left(n_{z} m 2_{y}+n_{y} m 2_{z}\right)=0 .
\end{aligned}
$$


As equaçoes $(1.23),(1.24)$ e (1.25) formant as condiçoes de contorno para as velociclades e a pressão na superfície livre. Para a temperatura utiliza-se a condigũo de fronteira de Robin em que o fluxo de calor $q=-k \nabla T \cdot \mathbf{n}$ é proporcional a diferenca de temperatura entre a superfície e o fluido. Assim

$$
-r \nabla T \cdot \mathbf{n}=h\left(T-T_{A}\right)
$$

cm que $\kappa$ é a condutividade térmica, h ó o cooficiente de transfêrencia de calor e $T_{A}$ a temperatura de referência na superfície livre.

\subsubsection{Condições no Contorno Rígido}

As condicões de não escorregamento "no-slip" e injeçäo "prescribed inflow" compòm as condições de contorno na superfície rígida. Essas condiçōes de contorno sào aplicadis na fronteira delimitada pelo contomo rígido (recipientes e injetores) em contato com o fluido. Assim, a velocidade tangencial e normal do fluido em relação à paredo sólida sâo definidas iguais à zero.

Para a temperatura é utilizada a condição adiabática, isto ó, o fluxo de calor é nulo entre a parede do recipiente e o fluido, on a condição de Dirichlet, isto é, a temperatura é prescrita na fronteira.

\subsection{Adimensionalização das equações}

Adimensionalizando as equações (1.1), (1.12), (1.15), (1.16), (1.17), (1.18), (1.19). (1.23), (1.24) e (1.25) surgem as grandezas adimensionais chamadas de número de Reynolds, Froude, Prandtl e Nusselt. Definindo as variáveis adimensionais pelas transformaçoes (Ferreira et al., 1999; Griebel et al., 1997)

$$
\begin{gathered}
\mathbf{u}=U \mathbf{u}^{*}, \mathbf{x}=L \mathbf{x}^{*}, \nu(T)=\nu_{0} \nu^{*}(T), p=\rho_{\infty} U^{2} p^{*}, \rho=\rho^{*} \rho_{\infty} \\
t=\frac{L}{U} t^{*}, \mathbf{g}=g \mathbf{g}^{*}, T=T^{*} \Delta T+T_{\infty}, \beta=\beta^{*} \Delta T
\end{gathered}
$$

em que $U, L, \nu_{0}$ e $\rho_{\infty}$ são os valores de referencia para o comprimento, velocidarle, viscosidade e a massa específica respectivamente, $\Delta T-T_{\text {max }}-T_{\text {min }}$ a diferença entie as temperaturas máxima e mínima no escoamento em que $T_{m i n}$ ó a temperatura mínima 
do escoamento e $T_{\text {max }}$ a temperatura máxima no escoamento, $T_{\infty}$ uma temperatura de refêrencia, a qual é tomada como $T_{\text {min }}{ }^{3}$.

Substituindo-se essas transformaçoes, desconsiderando o símbolo "**" por simplicidade, nas equações (1.1), (1.12), (1.15), (1.16), (1.17), (1.18), (1.19), (1.23), (1.24) e (1.25), obtém-se a forma adimensional das equações

- equação da conscrvalyăo de massa:

$$
\frac{\partial u}{\partial x}+\frac{\partial v}{\partial y}+\frac{\partial w}{\partial z}=0
$$

- equações de conservação de quantidado de movimento:

$$
\begin{aligned}
& \frac{\partial u}{\partial t}+\frac{\partial\left(u^{2}\right)}{\partial x}+\frac{\partial(u v)}{\partial y}+\frac{\partial(u w)}{\partial z}=-\frac{\partial p}{\partial x}+\frac{\nu(T)}{\operatorname{Re}}\left(\frac{\partial^{2} u}{\partial x^{2}}+\frac{\partial^{2} u}{\partial y^{2}}+\frac{\partial^{2} u}{\partial z^{2}}\right) \\
& +\frac{1}{\operatorname{Re}}\left[2 \frac{\partial u}{\partial x} \frac{\partial \nu(T)}{\partial x}+\left(\frac{\partial u}{\partial y}+\frac{\partial v}{\partial x}\right) \frac{\partial \nu(T)}{\partial y}+\left(\frac{\partial u}{\partial z}+\frac{\partial w}{\partial x}\right) \frac{\partial \nu(T)}{\partial z}\right] \\
& +(1-\beta T) \frac{1}{\operatorname{Fr}^{2}} g_{x} \\
& \frac{\partial v}{\partial t}+\frac{\partial(u v)}{\partial x}+\frac{\partial\left(v^{2}\right)}{\partial y}+\frac{\partial(v w)}{\partial z}=-\frac{\partial p}{\partial y}+\frac{\nu(T)}{\operatorname{Re}}\left(\frac{\partial^{2} v}{\partial x^{2}}+\frac{\partial^{2} v}{\partial y^{2}}+\frac{\partial^{2} v}{\partial z^{2}}\right) \\
& +\frac{1}{\operatorname{Re}}\left[\left(\frac{\partial u}{\partial y}+\frac{\partial v}{\partial x}\right) \frac{\partial \nu(T)}{\partial x}+2 \frac{\partial y}{\partial y} \frac{\partial u(T)}{\partial y}+\left(\frac{\partial w}{\partial y}+\frac{\partial v}{\partial z}\right) \frac{\partial \nu(T)}{\partial z}\right] \\
& +(1-\beta T) \frac{1}{\operatorname{Fr}^{2}} g_{y} \\
& \frac{\partial w}{\partial t}+\frac{\partial(u w)}{\partial x}+\frac{\partial(v w)}{\partial y}+\frac{\partial\left(w^{2}\right)}{\partial z}=-\frac{\partial p}{\partial z}+\frac{\nu(T)}{\operatorname{Re}}\left(\frac{\partial^{2} w}{\partial x^{2}}+\frac{\partial^{2} w}{\partial y^{2}}+\frac{\partial^{2} w}{\partial z^{2}}\right) \\
& +\frac{1}{\operatorname{Re}}\left[\left(\frac{\partial u}{\partial z}+\frac{\partial w}{\partial x}\right) \frac{\partial \nu(T)}{\partial x}+\left(\frac{\partial v}{\partial z}+\frac{\partial w}{\partial y}\right) \frac{\partial \nu(T)}{\partial y}+2 \frac{\partial w}{\partial z} \frac{\partial \nu(T)}{\partial z}\right] \\
& +(1-\beta T) \frac{1}{\mathrm{Fr}^{2}} g_{z}
\end{aligned}
$$

- equação da energia:

$$
\frac{\partial T}{\partial t}+\frac{\partial(u T)}{\partial x}+\frac{\partial(v T)}{\partial y}+\frac{\partial(w T)}{\partial z}=\frac{1}{\operatorname{RePr}}\left(\frac{\partial^{2} T}{\partial x^{2}}+\frac{\partial^{2} T}{\partial y^{2}}+\frac{\partial^{2} T}{\partial z^{2}}\right)
$$

\footnotetext{
${ }^{3}$ Quando se escolle $T_{\infty}=T_{m i n}$, tem-se $T^{*} \in[0,1]$.
} 
- modelos para o cálculo do $\nu(T)$.

$$
\begin{aligned}
& \nu(T)=\frac{1}{\nu_{0}}\left(r_{0} T^{2}+r_{1} T+r_{2}\right) \\
& \nu(T)=\frac{1}{\nu_{0}\left(c_{0} T^{2}+c_{1} T+r_{2}\right)}
\end{aligned}
$$

em que

$$
\begin{gathered}
c_{0}=a \Delta T^{2} \\
c_{1}-2 a T_{\infty} \Delta T+b \Delta T \\
c_{2}=a T_{\infty}^{\prime 2}+b T_{\infty}^{\prime}+c
\end{gathered}
$$

- equaçóes das condições de contorno na superfície livre:

$$
\begin{gathered}
p-\frac{2 \nu(T)}{R e}\left[\frac{\partial u}{\partial x} n_{x}^{2}+\frac{\partial n^{2}}{\partial y} n_{y}^{2} \frac{\partial w}{\partial z} n_{z}^{2}+\left(\frac{\partial u}{\partial y}+\frac{\partial v}{\partial x}\right) n_{x} n_{y}\right. \\
\left.+\left(\frac{\partial u}{\partial z}+\frac{\partial u}{\partial x}\right) n_{x} n_{z}+\left(\frac{\partial v}{\partial z}+\frac{\partial w}{\partial y}\right) n_{y} n_{z}\right]=0 \\
2 \frac{\partial u}{\partial x} m 1_{x} n_{x}+2 \frac{\partial v}{\partial y} m 1_{y} n_{y}+2 \frac{\partial w}{\partial z} m 1_{z} n_{z}+\left(\frac{\partial u}{\partial y}+\frac{\partial v}{\partial x}\right)\left(m 1_{x} n_{y}+m 1_{y} n_{x}\right) \\
+\left(\frac{\partial u}{\partial z}+\frac{\partial w}{\partial x}\right)\left(m 1_{x} n_{z}+m 1_{z} n_{x}\right)+\left(\frac{\partial v}{\partial z}+\frac{\partial w}{\partial y}\right)\left(m 1_{y} n_{z}+m 1_{z} n_{y}\right)=0 \\
2 \frac{\partial u}{\partial x} m 2_{x} n_{x}+2 \frac{\partial v}{\partial y} m 2_{y} n_{y}+2 \frac{\partial w}{\partial z} m 2_{z} n_{z}+\left(\frac{\partial u}{\partial y}+\frac{\partial v}{\partial x}\right)\left(m 2_{x} n_{y}+m 2_{y} n_{x}\right) \\
+\left(\frac{\partial u}{\partial z}+\frac{\partial w}{\partial x}\right)\left(m 2_{x} n_{z}+m 2_{z} n_{x}\right)+\left(\frac{\partial v}{\partial z}+\frac{\partial w}{\partial y}\right)\left(m 2_{y} n_{z}+m 2_{z} n_{y}\right)-0 \\
-\nabla T \cdot \mathbf{n}=\mathrm{Nu}\left(T-T_{A}\right)
\end{gathered}
$$




\section{Capítulo 2}

\section{Método Computacional}

Soluções analíticas para as equações de Navier-Stokes são determinadas para poucos casos. A dificuldarde de se encontrar soluçóes analíticas decorre do fato que as cquacoes de Navicr-Stokes são equaçoes diferenciais parciais nào-lineares, a a teoria matemática para essa classe de equaçoes ainda não está suficientemente desenvolvida para permitir a obtenção de soluçôs analíticas em domínios e condições gerais.

Portanto, desenvolveram-se métodos numéricos para a solução das equaçoes de NavierStokes, como os métodos GENSMAC ("Generalized-Simplified-Marker-and-Cell") (Tomó e McKee, 1994) e o GENSMAC-3D (extensão do método GENSMAC para três dimensões) (Tomé et. al., 2001).

\subsection{Método para o Cálculo da Velocidade, Pressão e Temperatura}

A seguir será apresentada uma adaptação do procedimento computacional contido no código GENSMAC-3D para a simulação de escoamentos não-isotérmicos. A idéia basica do método GENSMAC-3D é resolver as equaçóes de Navier-Stokes sobre uma malha diferenciada no tempo $t=t_{0}+\delta t$ utilizando partículas marcadoras para representar o fluido.

Suponhamos que em um dado tempo to o campo de velocidades $\mathbf{u}\left(\mathbf{x}, t_{0}\right)$ é conhecido e as condiçōes de fronteira para a velocidade e pressão sejam dadas. A temperatura $T\left(\mathbf{x}, t_{0}\right)$ 
também é conhecida e as suas condiçôes de fronteiras também sào dadas.

O campo de volocidade atualizado $\mathbf{u}(\mathbf{x}, t)$ e a temperatura $T(\mathrm{x}, t)$, onde $t=t_{0}+\delta t$ sào calculados como segue:

1. Seja $\bar{p}\left(\mathbf{x}, l_{0}\right)$ um campo de pressão que satisfaça a condiçäo de tensão normal correta sobre a superficie livre em $t=t_{1}$.

2. Calcula-se o campo de velocidade intermediário $\tilde{u}(\mathbf{x}, t)$ por meio das equaçós

$$
\begin{aligned}
& \frac{\partial \tilde{u}}{\partial t}=-\frac{\partial\left(u^{2}\right)}{\partial x}-\frac{\partial(u v)}{\partial y}-\frac{\partial(u w)}{\partial z}-\frac{\partial \tilde{p}}{\partial x}+\frac{\nu(T)}{\operatorname{Re}}\left(\frac{\partial^{2} u}{\partial x^{2}}+\frac{\partial^{2} u}{\partial y^{2}}+\frac{\partial^{2} u}{\partial z^{2}}\right) \\
& +\frac{1}{\operatorname{Re}}\left[2 \frac{\partial u}{\partial x} \frac{\partial \nu(T)}{\partial x}+\left(\frac{\partial u}{\partial y}+\frac{\partial u}{\partial x}\right) \frac{\partial \nu(T)}{\partial y}+\left(\frac{\partial u}{\partial z}+\frac{\partial u}{\partial x}\right) \frac{\partial \nu(T)}{\partial z}\right] \\
& +(1-\beta T) \frac{1}{\mathrm{Fr}^{2}} y_{x} \\
& \frac{\partial \tilde{v}}{\partial t}=\frac{\partial(u v)}{\partial x}-\frac{\partial\left(v^{2}\right)}{\partial y}-\frac{\partial(v w)}{\partial z}-\frac{\partial \tilde{p}}{\partial y}+\frac{\nu(T)}{\operatorname{Re}}\left(\frac{\partial^{2} v}{\partial x^{2}}+\frac{\partial^{2} v}{\partial y^{2}}+\frac{\partial^{2} v}{\partial z^{2}}\right) \\
& +\frac{1}{\operatorname{Re}}\left[\left(\frac{\partial u}{\partial y}+\frac{\partial v}{\partial x}\right) \frac{\partial \nu(T)}{\partial x}+2 \frac{\partial v}{\partial y} \frac{\partial \nu(T)}{\partial y}+\left(\frac{\partial w}{\partial y}+\frac{\partial v}{\partial z}\right) \frac{\partial \nu(T)}{\partial z}\right] \\
& +(1-\beta T) \frac{1}{\mathrm{Fr}^{2}} g_{y} \\
& \frac{\partial \tilde{w}}{\partial t}=-\frac{\partial(u w)}{\partial x}-\frac{\partial(v w)}{\partial y}-\frac{\partial\left(w^{2}\right)}{\partial z}-\frac{\partial \tilde{p}}{\partial z}+\frac{\nu(T)}{\operatorname{Re}}\left(\frac{\partial^{2} w}{\partial x^{2}}+\frac{\partial^{2} w}{\partial y^{2}}+\frac{\partial^{2} w}{\partial z^{2}}\right) \\
& +\frac{1}{\operatorname{Re}}\left[\left(\frac{\partial u}{\partial z}+\frac{\partial w}{\partial x}\right) \frac{\partial \nu(T)}{\partial x}+\left(\frac{\partial v}{\partial z}+\frac{\partial w}{\partial y}\right) \frac{\partial \nu(T)}{\partial y}+2 \frac{\partial w}{\partial z} \frac{\partial \nu(T)}{\partial z}\right] \\
& +(1-\beta T) \frac{1}{\mathrm{Fr}^{2}} g_{z}
\end{aligned}
$$

$\operatorname{com} \tilde{\mathbf{u}}\left(\mathbf{x}, t_{0}\right)=\mathbf{u}\left(\mathbf{x}, t_{0}\right)$, utilizando a condiçăo de fronteira correta para $\mathbf{u}\left(\mathbf{x}, t_{0}\right)$. As equaçoes (2.1) - (2.3) sĩo resolvidas utilizando-se aproximaçio por diferenças finitas. Na forma vetorial, as equacoes (2.1) - (2.3) podem ser escritas como

$$
\frac{\partial \tilde{\mathbf{u}}}{\partial t}--\nabla \tilde{p}+N(\mathbf{u})
$$

com 


$$
\begin{aligned}
& N_{1}=-\frac{\partial\left(u^{2}\right)}{\partial x}-\frac{\partial(u v)}{\partial y}-\frac{\partial(u w)}{\partial z}+\frac{\mu(T)}{\operatorname{Re}}\left(\frac{\partial^{2} u}{\partial x^{2}}+\frac{\partial^{2} u}{\partial y^{2}}+\frac{\partial^{2} u}{\partial z^{2}}\right) \\
& +\frac{1}{R v}\left[2 \frac{\partial u}{\partial x} \frac{\partial \nu(T)}{\partial x}+\left(\frac{\partial u}{\partial y}+\frac{\partial v}{\partial x}\right) \frac{\partial \nu(T)}{\partial y}+\left(\frac{\partial u}{\partial z}+\frac{\partial u}{\partial x}\right) \frac{\partial \nu(T)}{\partial z}\right] \\
& +(1-\beta T) \frac{1}{\mathrm{Fr}^{2}} g_{x} \\
& N_{2}=-\frac{\partial(u v)}{\partial x}-\frac{\partial\left(v^{2}\right)}{\partial y}-\frac{\partial(u w)}{\partial z}+\frac{\nu(T)}{\mathrm{R} e}\left(\frac{\partial^{2} v}{\partial x^{2}}+\frac{\partial^{2} v}{\partial y^{2}}+\frac{\partial^{2} u}{\partial z^{2}}\right) \\
& +\frac{1}{\operatorname{Re}}\left[\left(\frac{\partial u}{\partial y}+\frac{\partial v}{\partial x}\right) \frac{\partial \nu(T)}{\partial x}+2 \frac{\partial v}{\partial y} \frac{\partial \nu(T)}{\partial y}+\left(\frac{\partial w}{\partial y}+\frac{\partial v}{\partial z}\right) \frac{\partial \nu(T)}{\partial z}\right] \\
& +(1-\beta T) \frac{1}{\mathrm{Fr}^{2}} y_{y}
\end{aligned}
$$

c

$$
\begin{aligned}
& N_{3}=-\frac{\partial(u w)}{\partial x}-\frac{\partial(v w)}{\partial y}-\frac{\partial\left(w^{2}\right)}{\partial z}+\frac{\nu(T)}{\operatorname{Re}}\left(\frac{\partial^{2} w}{\partial x^{2}}+\frac{\partial^{2} w}{\partial y^{2}}+\frac{\partial^{2} w}{\partial z^{2}}\right) \\
& +\frac{1}{\operatorname{Re}}\left[\left(\frac{\partial u}{\partial z}+\frac{\partial w}{\partial x}\right) \frac{\partial \nu(T)}{\partial x}+\left(\frac{\partial v}{\partial z}+\frac{\partial w}{\partial y}\right) \frac{\partial \nu(T)}{\partial y}+2 \frac{\partial w}{\partial z} \frac{\partial \nu(T)}{\partial z}\right] \\
& +(1-\beta T) \frac{1}{\operatorname{Fr}^{2}} g_{z}
\end{aligned}
$$

Escrevendo as equações (1.28)-(1.30) na forma vetorial podemos escrever

$$
\frac{\partial \mathbf{u}}{\partial t}=-\nabla p+N(\mathbf{u})
$$

Subtraindo (2.4) de (2.5) tem-se

$$
\frac{\partial}{\partial t}(\mathbf{u}-\tilde{\mathbf{u}})=-\nabla(p-\tilde{p})
$$

Aplicando-se o operador rotacional a ambos os lados de (2.6) obtém-so

$$
\nabla \times\left[\frac{\partial}{\partial t}(\mathbf{u}-\tilde{\mathbf{u}})\right]-\nabla \times[-\nabla(p-\tilde{p})]
$$

donde vem

$$
\nabla \times\left[\frac{\partial}{\partial t}(\mathbf{u}-\tilde{\mathbf{u}})\right]=0
$$


Escrevendo (2.7) como

$$
\frac{\partial}{\partial l}[\nabla \times(\mathbf{u}-\tilde{\mathbf{u}})]=0
$$

entãa pode-se dizer que

$$
\nabla \times(\mathbf{u}-\tilde{\mathbf{u}})=f(x)
$$

para qualquer $f(x)$ com $t \in\left[t_{0}, t_{0}+\delta t\right]$.

Sendo $\mathbf{u}=\overrightarrow{\mathbf{u}}$ em $t=t_{0}$, temos que $\nabla \times \mathbf{u}=\nabla \times \tilde{\mathbf{u}}$ em $t-t_{0}$, o que implica cque $f(x)=0$.

Assim

$$
\nabla \times(\mathbf{u}-\tilde{\mathbf{u}})=0
$$

para qualquer $t \in\left[t_{0}, t_{0}+\delta t\right]$.

Assim as vorticidades associadas a $\mathbf{u}$ e $\tilde{\mathbf{u}}$ são iguais. Porém $\tilde{\mathbf{u}}$ não satisfaz $\nabla \cdot \tilde{\mathbf{u}}=0$. Seja $\psi(\mathbf{x}, t)$ uma função escalar tal que

$$
\mathbf{u}(\mathbf{x}, t)=\ddot{\mathbf{u}}(\mathbf{x}, t)-\nabla \psi(\mathbf{x}, t) .
$$

Aplicando o divergente em ambos os lados de (2.8) obtém-se

$$
\nabla \cdot \mathbf{u}(\mathbf{x}, t)=\nabla \cdot \tilde{\mathbf{u}}(\mathbf{x}, t) \cdots \nabla^{2} \ell^{\prime}(\mathbf{x}, t)
$$

Impondo-se a conservação de massa obtém-se a equação de Poisson para a função $\psi(\mathbf{x}, t)$

$$
\nabla^{2} \psi(\mathbf{x}, t)=\nabla \cdot \tilde{\mathbf{u}}(\mathbf{x}, t)
$$

3. Resolve-se a equação de Poisson

$$
\nabla^{2} \psi(\mathrm{x}, t)=\nabla \cdot \check{\mathrm{u}}(\mathrm{x}, t)
$$


As condições de fronteira para $\psi$ sào

$$
\begin{aligned}
& \frac{\partial \psi}{\partial n}=0 \quad \text { sobre a fronteira rígida } \\
& \psi=0 \quad \text { sobre a superfície livro }
\end{aligned}
$$

onde n é a direção normal ao contomo rígido. A discretização da equação de Poisson gera um sistema linear que será resolvido pelo método dos gradientes conjugados.

4. Calcular o (ammon de velocidade atualizado por

$$
\mathbf{u}(\mathbf{x}, t)=\tilde{\mathbf{u}}(\mathbf{x}, t)-\nabla \psi(\mathbf{x}, t)
$$

5. Calcular a nova pressão. A pressão atualizada é calculada cono segue. Substituindo (2.11) em (2.6), obtém-se

$$
\frac{\partial(-\nabla(i)}{\partial t}=-\nabla(p-\tilde{p})
$$

Então

$$
\frac{\partial(\psi \cdot(\mathbf{x}, t))}{\partial t}=(p-\tilde{p})
$$

que pode ser aproximada por

$$
\frac{\psi(\mathbf{x}, t)-\psi\left(\mathbf{x}, t_{0}\right)}{\delta t}=p-\tilde{p}
$$

Sendo $\mathbf{u}\left(\mathbf{x}, t_{0}\right)=\ddot{\mathbf{u}}\left(\mathbf{x}, t_{0}\right)$ e através de (2.11) tem-se

$$
\nabla\left(\mathbf{x}, t_{0}\right)=0
$$

implicando cue $\psi\left(\mathrm{x}, t_{0}\right)$ é constante. Como $\psi=0$ na superfície livre $\psi\left(\mathbf{x}, t_{0}\right)=0$ então a equação (2.14) torna-se

$$
p=\tilde{p}+\frac{\psi(\mathrm{x}, t)}{\delta l}
$$


em que di ć o tamanho do passo no tempo.

6. Calcula-se $T(\mathbf{x}, t)$ e $\nu(T(\mathbf{x}, t))$ usando a equação da energia (1.31).

7. Mover a superficio livre. Este último passo implica o movimento das partírulas marcadoras para suas novas posiçöes. Essas partículas são gerarlas nos injetores o injetadas no domínio, permitindo assim uma visualização do escoamento e obtenção da orientação da superfício livre. As coordenadas das partículas virtuais são armazenadas a cada ciclo de cálculo e, atualizadas resolvendo-so o PVI

$$
\frac{d \mathrm{x}}{d t}=\mathrm{u}, \quad \mathrm{x}\left(t_{0}\right)=\mathrm{x}_{0}
$$

pelo método de Euler, fornecendo assim as novas coordenadas das partículas. Isso determina se uma partícula se nove para dentro de uma célnla on deixa a regiào do domínio atravós de um ejetor.

\subsection{Modificações no Método GENSMAC-3D}

Para a implementação do método descrito na secção anterior, fez-se algumas modificaçòes no método GENSMAC-3D, ou seja, foi acrescentado o cálculo de $T(\mathbf{x}, t)$ e $\nu(T(\mathbf{x}, t))$ e feito o ajuste das equações de Navior-Stokes para que simulaçōes com influência da temperatura possam ser feitas. O método acima também foi ajustado para permitir simulaçoes em que a viscosidade é constante. 


\section{Capítulo 3}

\section{Discretizações}

As cquações desenvolvidas anteriormente foram incorporadas no ambiente de simulaçăo FreeFlow-3D. Este capítulo mostra as discretizaçoes das equaçoes para escoamentos não-isotérmicos. 'Também são apresentadas técnicas para o tratamento das condiçoes de contorno na superfície livre e no contorno rígido.

\subsection{Definição das Células}

Como o fluido está continuamente em movimento faz-se necessário utilizar um procedimento de identificação da região que contém o fluido o a superfície livre. Para isto, identifica-se todas as cólulas computacionais. As células podern ser

- Injetor/"Inflow" (I): células que definem a entrada do fluido na regiăo do romínio;

- Fronteira/"Boundary" (B): células que definem o contorno rígido se possuem metade on mais de seu volume no contorno rígido, de modo que possam ser impostas as condições de contorno;

- Vazia/ "Empty" (E): células que năo contêm fluido;

- Superfície/"Surface" (S): células que contêm fluido com pelo menos uma célula vizinha vazia e definem a posição da superfície livre:

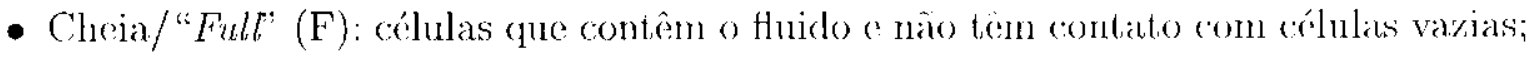


- Ejetor/"Outflow" (O): cólulas que simulam a saída do fluido da região cle rlonínio.

Para visualizar melhor a identificação das células, ver a figura 3.1. Para a simplificação no entendimento da figura, optou-se pela supressão da nomenclatura das células vazias (E).

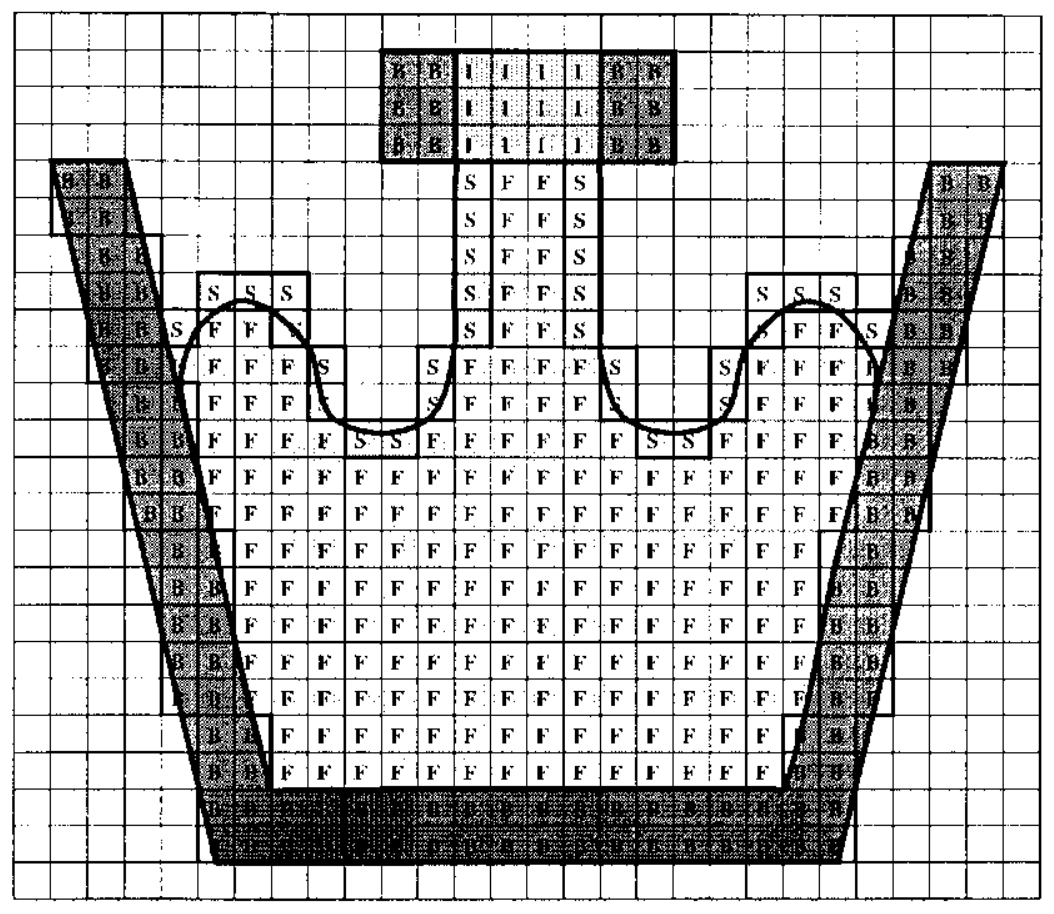

Figura 3.1: Tipo de células.

Em geral, durante a simulação do escoamento, uma mesma cólula pode ter seu estado alterado, isto é, passando de uma célula (E) para (S), ou de (S) para (F) por exemplo.

\subsection{Discretização do Domínio}

A discretização das equaçoes será feita pelo método de diferenças finitias numa malhal diferenciada tridimensional. A malha é uniformemente espacada (mo caula direcäo, trendo as cólulas comprimento $\delta x$, largura $\delta y$ o altura $\delta z$. As volocidades são definidas nas faces das células, a pressĩo $(p)$, a temperatura $(T)$ e a função potencial $(\psi)$ em seu centro como pode ser visto na figura 3.2 . 


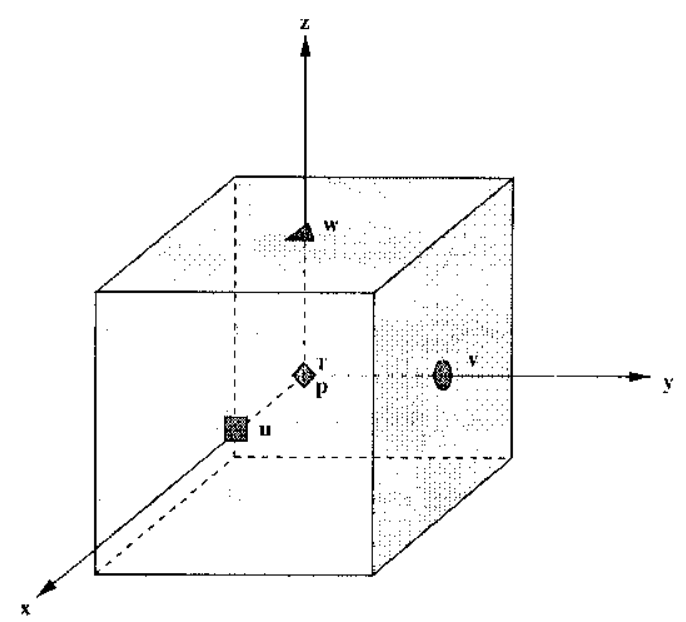

Figura 3.2: Célula computacional, mostrando onde as variáveis são discretizadas.

\subsection{Discretização das Equações de Conservação de Quantidade de Movimento}

A equação de quantidade de movimento na direção $x$ será discretizada na posição $\left(i+\frac{1}{2}, j, k\right)$, na diregão $y$ será discretizada na posição $\left(i, j+\frac{1}{2}, k\right)$ o na direção $z$ na posição $\left(i, j, k+\frac{1}{2}\right)$. As derivadas temporais são discretizadas utilizando-se Euler explícito, as derivadas espaciais utilizando-se diferenças centrais.

Considere a equacão de conservaço de quantidade de movimento adimensional na dirçào $x$ dada por

$$
\begin{aligned}
& \frac{\partial u}{\partial t}+\frac{\partial\left(u^{2}\right)}{\partial x}+\frac{\partial(u v)}{\partial y}+\frac{\partial(u u)}{\partial z}=-\frac{\partial p}{\partial x}+\frac{\nu(T)}{\operatorname{Re}}\left(\frac{\partial^{2} u}{\partial x^{2}}+\frac{\partial^{2} u}{\partial y^{2}}+\frac{\partial^{2} u}{\partial z^{2}}\right) \\
& +\frac{1}{\operatorname{Re}}\left[2 \frac{\partial u}{\partial x} \frac{\partial \nu(T)}{\partial x}+\left(\frac{\partial u}{\partial y}+\frac{\partial v}{\partial x}\right) \frac{\partial \nu(T)}{\partial y}+\left(\frac{\partial u}{\partial z}+\frac{\partial u}{\partial x}\right) \frac{\partial \nu(T)}{\partial z}\right] \\
& \left.+(1-\beta T) \frac{1}{\mathrm{H}^{2} g^{2}}\right]
\end{aligned}
$$

Na equação (3.1), a derivada temporal e o gradiente de pressäo são aproximadas por

$$
\begin{aligned}
& \left.\frac{\partial u}{\partial t}\right|_{i+\frac{1}{2}, j, k} \approx \frac{u_{i+\frac{1}{2}, j, k}^{n+1}-u_{i+\frac{1}{2}, j, k}^{n}}{\delta l} \\
& \left.\frac{\partial p}{\partial x}\right|_{i+\frac{1}{2}, j, k} \approx \frac{p_{i+1, j, k}-p_{i, j, k}}{\delta r}
\end{aligned}
$$


As derivadas dos termos convectivos são aproximadas por

$$
\begin{gathered}
\left.\frac{\partial u^{2}}{\partial x}\right|_{i+\frac{1}{i}, j, k} \approx \frac{u_{i+1, j, k}^{2} \cdot u_{i, j, k}^{2}}{\delta x} \\
\left.\frac{\partial(u v)}{\partial y}\right|_{i+\frac{1}{2}, j, k} \approx \frac{(u v)_{i+\frac{1}{2}, j+\frac{1}{2}, k}-(u v)_{i+\frac{1}{2}, j-\frac{1}{2}, k}}{\delta y} \\
\left.\frac{\partial(w u)}{\partial z}\right|_{i+\frac{1}{2}, j, k} \approx \frac{(w u)_{i+\frac{1}{2}, j, k+\frac{1}{3}}-(w u)_{i+\frac{1}{2}, j, k \quad}}{\delta z}
\end{gathered}
$$

sendo que em $(3.4)-(3.6)$ os termos $u_{i+1, j, k}^{2}, u_{i, j, k}^{2}(u v)_{i+\frac{1}{2}, j+\frac{1}{2}, k},(u v)_{i+\frac{1}{2}, j-\frac{1}{2}, k}$ e $(u v)_{i} \frac{1}{2, j+\frac{1}{2}, k}$ não sầo pontos de discretização na malha, devendo assim ser obtidos por interpolação usando um esquema de alta ordem tal como o VONOS, que será visto na seçäo 3.j.

As derivadas dos termos viscosos são aproximadas por

$$
\begin{gathered}
\left.\frac{\partial^{2} u}{\partial x^{2}}\right|_{i+\frac{1}{2}, j, k} \approx \frac{u_{i-\frac{1}{2}, j, k}-2 u_{i+\frac{1}{2}, j, k}+u_{i+\frac{3}{2}, j, k}}{\delta x^{2}} \\
\left.\frac{\partial^{2} u}{\partial y^{2}}\right|_{i+\frac{1}{2}, j, k} \approx \frac{u_{i+\frac{1}{2}, j-1, k}-2 u_{i+\frac{1}{2}, j, k}+u_{i+\frac{1}{2}, j+1, k}}{\delta y^{2}} \\
\left.\frac{\partial^{2} u}{\partial z^{2}}\right|_{i+\frac{1}{2}, j, k} \approx \frac{u_{i+\frac{1}{2}, j, k-1}-2 u_{i+\frac{1}{2}, j, k}+u_{i+\frac{1}{2}, j, k+1}}{\delta z^{2}} \\
\left.\frac{\partial u}{\partial x}\right|_{i+\frac{1}{2}, j, k} \approx \frac{u_{i+\frac{3}{2}, j, k}-u_{i, \frac{1}{2}, j, k}}{2 \delta x} \\
\left.\frac{\partial u}{\partial y}\right|_{i+\frac{1}{2}, j, k} \approx \frac{u_{i+\frac{3}{2}, j+1, k}-u_{i+\frac{1}{2}, j-1, k}}{2 \delta y} \\
\left.\frac{\partial v}{\partial x}\right|_{i-\frac{1}{2}, j, k} \approx \frac{u_{i+1, j, k}-v_{i, j, k}}{\delta x}
\end{gathered}
$$

sendo que em $(3.12)$

$$
v_{i, j, k}=\frac{v_{i, j+\frac{1}{2}, k}+v_{i, j-\frac{1}{2}, k}}{2}
$$

o

$$
\begin{gathered}
v_{i+1, j, k}=\frac{v_{i-1, j+\frac{1}{2}, k}+v_{i+1, j-\frac{1}{2}, k}}{2} \\
\left.\frac{\partial u}{\partial z}\right|_{i+1, j, k} \approx \frac{u_{i+\frac{1}{2}, j, k+1}-u_{i+\frac{1}{2}, j, k-1}}{2 \delta ;} \\
\left.\frac{\partial w}{\partial x}\right|_{i-\frac{1}{2}, j, k} \approx \frac{w_{i+1, j, k}-w_{i, j, k}}{\delta x}
\end{gathered}
$$


sendo que em (3.14)

$$
w_{i, j, k}=\frac{w_{i, j, k+\frac{1}{3}}+w_{i, j, k-\frac{1}{2}}}{2}
$$

(?.

$$
w_{i+1, j, k}=\frac{w_{i+1, j, k-1, \frac{1}{2}}+w_{i+1, j, k-\frac{1}{2}}}{2} .
$$

As derivadas de $\nu(T)$ são aproximadas por

$$
\begin{gathered}
\left.\frac{\partial \nu(T)}{\partial x}\right|_{i+\frac{1}{2}, j, k} \approx \frac{\nu\left(T_{i+1, j, k}\right)-\nu\left(T_{i, j, k}\right)}{\delta x} \\
\left.\frac{\partial \nu(T)}{\partial y}\right|_{i+\frac{1}{2}, j, k} \approx \frac{\nu\left(T_{i+\frac{1}{2}, j+\frac{1}{2}, k}\right)-\nu\left(T_{i+\frac{1}{2}, j-\frac{1}{2}, k}\right)}{\delta y}
\end{gathered}
$$

serido que

$$
T_{i+\frac{1}{2}, j+\frac{1}{2}, k}=\frac{T_{i, j, k}+T_{i+1, j, k}+T_{i, j+1, k}+T_{i|1, j| 1, k}}{4}
$$

$$
I_{i+\frac{1}{2}, j-\frac{1}{2}, k}^{\prime}=\frac{T_{i, j, k}+T_{i+1, j, k}+T_{i, j-1, k}+T_{i+1, j-1, k}}{4} .
$$

O) termo fonte ć aproximado por

$$
\left.(1-\beta T) \frac{1}{\mathrm{Fr}^{2}} g_{x}\right|_{i+\frac{1}{2}, j, k} \approx\left(1-\beta T_{i+\frac{1}{2}, j, k}\right) \frac{1}{\mathrm{Fr}^{2}} g_{x}
$$

sendo que

$$
T_{i+\frac{1}{2}, j, k}=\frac{T_{i+1, j, k}+T_{i, j, k}}{2}
$$

As discretizações das equações de quantidade de movimento nas dircçoos y a z são obtidas de maneira análoga.

\subsection{Discretização da Equação da Energia}

A equação da energia será discretizada na posição $(i, j, k)$. Do mesmo modo como foi feito para as equações de quantidade de movimento, a derivada temporal será discretizada utilizando-se diferenças progressivas e as derivadas espaciais utilizando-se diferenças 
rentrais.

A discretizaçào da derivada temporal ó dada por

$$
\left.\frac{\partial T}{\partial t}\right|_{i, j, k} \approx \frac{T_{i, j, k}^{n+1}-T_{i, j, k}^{n}}{\delta t}
$$

Discretizando-se as derivadas espaciais temos

$$
\begin{aligned}
& \left.\frac{\partial(u T)}{\partial x}\right|_{i, j, k} \approx \frac{(u T)_{i+\frac{1}{2}, j, k}-(u T)_{i-\frac{1}{2}, j, k}}{\delta x} \\
& \left.\frac{\partial(v T)}{\partial y}\right|_{i, j, k} \approx \frac{(u T)_{i, j+\frac{1}{2}, k}-(v T)_{i, j-\frac{1}{2}, k}}{\delta y} \\
& \left.\frac{\partial(w T)}{\partial z}\right|_{i, j, k} \approx \frac{(w T)_{i, j, k+\frac{1}{2}}-(u T)_{i, j, k-\frac{1}{2}}}{\delta z} \\
& \left.\frac{\partial^{2} T}{\partial x^{2}}\right|_{i, j, k} \approx \frac{T_{i+1, j, k}-2 T_{i, j, k}+T_{i-1, j, k}}{\delta x^{2}} \\
& \left.\frac{\partial^{2} T}{\partial y^{2}}\right|_{i, j, k} \approx \frac{T_{i, j, 1, k}-2 T_{i, j, k}+T_{i, j-1, k}}{\delta y^{2}} \\
& \left.\frac{\partial^{2} T}{\partial z^{2}}\right|_{i, j, k} \approx \frac{T_{i, j, k+1}-2 T_{i, j, k}+T_{i, j, k-1}}{\delta z^{2}}
\end{aligned}
$$

Os termos $(u T)_{i \pm \frac{1}{2}, j, k},(v T)_{i, j \pm \frac{1}{2} k}$ c $(u T)_{i, j . k \pm_{2}^{1}}$ não são pontos de discretização na malha devendo assim ser obtidos usando o mesmo procedimento utilizado em (3.4)-(3.6).

\subsection{Aproximação dos Termos Convectivos}

Os termos convectivos serão tratados de maneira particular por serem os principais causadores de instabilidade numérica nas simulacões, portanto a discretização arlequada para os termos convectivos é de extrema importância para a qualidade da solução numérica. Para se aproximar esses termos nas equaçoes de Navier-Stokes existem vários esquemas de interpolação. Nesta secção apresentam-se formas para se aproximar essises termos. Dependendo dos esquemas de interpolação utilizados, diferentes expressoes para os termos convectivos são possíveis.

Considere a figura 3.3 para aproximar a derivada parcial de uma variável genérica $\phi$ no pouto $P_{0}$, ou seja, $\frac{\partial \phi}{\partial s}$, em que s representa uma direça $x, y$ ou $z 0, \phi_{A}, \phi_{B}$ são os valores 


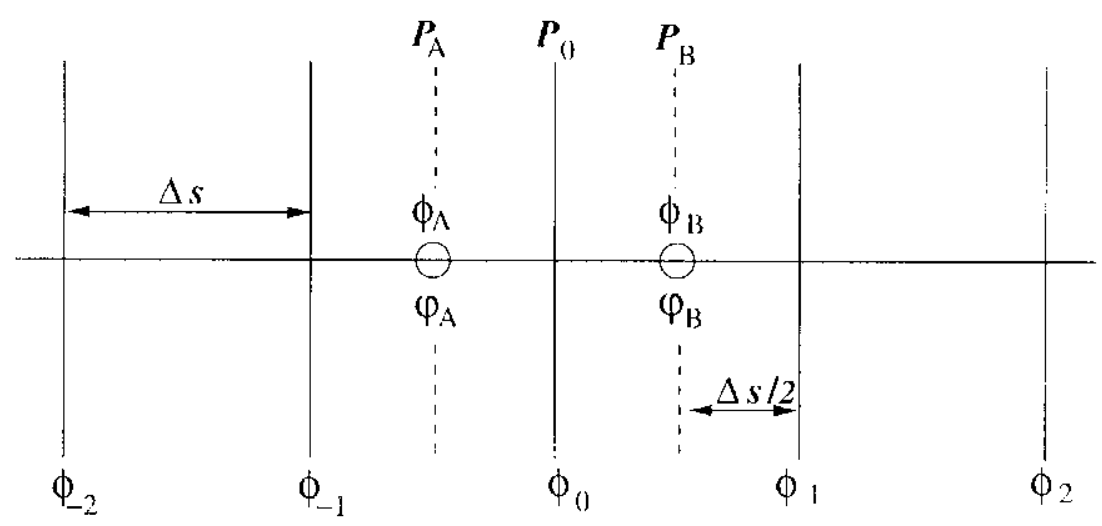

Figura 3.3: Estêncil usado para calcular $\phi_{A}$ e $\phi_{B 3}$ 11sando vários esquemas.

das variáveis genéricas nos pontos $P_{A}$ \& $P_{B}$ respectivamente, e $\varphi_{A}, \varphi_{B}$ são os valores da velocidade de conveçio nos pontos $P_{A}$ e $P_{B}$ respectivamente. Portanto a derivada

$$
\left.\frac{\partial \phi}{\partial s}\right|_{P_{0}}=\frac{\phi_{A}-\phi_{B}}{\Delta s}
$$

pode ser obtida usando um dos esquemas abaixo (Ferreira et al., 20022). ()s valores de $\phi_{A}$ e $\phi_{B}$ são obtidos em termos dos valores vizinhos, $\phi_{-2}, \phi_{-1}, \phi_{0}, \phi_{1}, \phi_{2}$ e das velocidades convectivas $\varphi_{A}: \varphi_{B}$.

- UPO ("Upwind" de Primeira Ordem):

$$
\phi_{B 3}=\left\{\begin{array}{ll}
\phi_{0} & \text { se } \varphi_{B} \geq 0 \\
\phi_{1} & \text { se } \varphi_{B}<0,
\end{array} \quad \phi_{A}= \begin{cases}\phi_{-1} & \text { se } \varphi_{A} \geq 0 \\
\phi_{0} & \text { se } \varphi_{A}<0\end{cases}\right.
$$

- DC (Diferenças Centrais):

$$
\phi_{B}=\frac{\phi_{1}+\phi_{0}}{2}, \quad \phi_{A}-\frac{\phi_{0}+\phi_{-1}}{2} .
$$

- QUICK ("Quadratic Upstream Interpolation for Convective Kinematics"):

$$
\phi_{B}= \begin{cases}\frac{3}{8} \phi_{1}+\frac{6}{8} \phi_{0}-\frac{1}{8} \phi_{-1} & \text { se } \varphi_{B} \geq 0 \\ \frac{3}{8} \phi_{0}+\frac{6}{8} \phi_{1}-\frac{1}{8} \phi_{2} & \text { se } \varphi_{B}<0\end{cases}
$$




$$
\phi_{A}= \begin{cases}\frac{3}{8} \phi_{0}+\frac{6}{8} \phi_{1} \ldots \frac{1}{8} \phi_{-2} & \text { se } \varphi_{A} \geq 0 \\ \frac{3}{8} \phi_{-1}+\frac{6}{8} \phi_{0}-\frac{1}{8} \phi_{1} & \text { se } \varphi_{A}<0\end{cases}
$$

- HLPA ("Iybrid-Linear Parabolic Approximation"):

$$
\begin{aligned}
& \text { se } \varphi_{B} \geq 0, \quad \dot{\phi}_{B}= \begin{cases}\dot{\phi}_{0} & \text { se } \hat{\phi}_{B} \notin[0,1] \\
\dot{\phi}_{0}+\left(\dot{\phi}_{1}-\phi_{0}\right) \hat{\dot{\phi}}_{B} & \text { se } \hat{\phi}_{B} \in[0,1]\end{cases} \\
& \text { se } \varphi_{B}<0, \quad \phi_{B}- \begin{cases}\hat{\phi}_{1} & \text { se } \hat{\phi}_{B} \notin[0,1] \\
\phi_{1}+\left(\phi_{0}-\phi_{1}\right) \hat{\phi}_{B} & \text { se } \hat{\phi}_{B} \subset[0,1],\end{cases} \\
& \text { se } \varphi_{A} \geq 0, \quad \phi_{A}- \begin{cases}\phi_{-1} & \text { se } \hat{\phi}_{A} \notin[0,1] \\
\phi_{-1}+\left(\phi_{0}-\phi_{-1}\right) \dot{\phi}_{A} & \text { se } \hat{\phi}_{A} \in[0,1],\end{cases} \\
& \text { se } \varphi_{A}<0, \quad \dot{\phi}_{A}= \begin{cases}\phi_{0} & \text { se } \hat{\phi}_{A} \not \subset[0,1] \\
\phi_{0}+\left(\phi_{-1}-\phi_{0}\right) \hat{\phi}_{A} & \text { se } \hat{\phi}_{A} \in[0,1] .\end{cases}
\end{aligned}
$$

- VONOS ("Variable-Order Non-Oscillatory Scheme"):

$$
\begin{aligned}
& \text { se } \varphi_{B} \geq 0, \quad \dot{\phi}_{B}= \begin{cases}\phi_{0} & \text { se } \hat{\phi}_{B} \notin[0,1] \\
10 \phi_{0}-9 \phi_{-1} & \text { se } \hat{\phi}_{B} \in[0,3 / 74) \\
\frac{3}{8} \phi_{1}+\frac{6}{8} \phi_{0}-\frac{1}{8} \phi_{-1} & \text { se } \hat{\phi}_{B} \in[3 / 74,1 / 2) \\
1.5 \phi_{0}-0.5 \phi_{-1} & \text { se } \hat{\phi}_{B} \in[1 / 2,2 / 3) \\
\phi_{1} & \text { se } \hat{\phi}_{B} \in[2 / 3,1],\end{cases} \\
& \text { se } \varphi_{B}<0, \quad \phi_{B}= \begin{cases}\phi_{1} & \text { se } \hat{\phi}_{B} \notin[0,1] \\
10 \phi_{1}-9 \phi_{2} & \text { se } \hat{\phi}_{B} \in[0,3 / 74) \\
\frac{3}{8} \phi_{0}+\frac{6}{8} \phi_{1}-\frac{1}{8} \phi_{2} & \text { se } \hat{\phi}_{B} \in[3 / 74,1 / 2) \\
1.5 \phi_{1}-0.5 \phi_{2} & \text { se } \hat{\phi}_{B} \in[1 / 2,2 / 3) \\
\phi_{0} & \text { se } \hat{\phi}_{B} \in[2 / 3,1],\end{cases}
\end{aligned}
$$




$$
\begin{aligned}
& \text { se } \varphi_{A} \geq 0, \quad \dot{\phi}_{A}= \begin{cases}\phi_{-1} & \text { se } \hat{\phi}_{A} \notin[0,1] \\
10 \phi_{-1}-9 \phi_{-2} & \text { se } \hat{\phi}_{A} \in[0,3 / 74) \\
\frac{3}{8} \phi_{0}+\frac{i}{8} \phi_{-1}-\frac{1}{8} \phi_{2} 2 & \text { se } \hat{\phi}_{A} \in[3 / 74,1 / 2) \\
1 . \bar{j} \phi_{-1}-0.5 \phi_{-2} & \text { se } \hat{\phi}_{A} \subset[1 / 2,2 / 3) \\
\phi_{0} & \text { se } \hat{\phi}_{A} \in[2 / 3,1],\end{cases} \\
& \text { se } \varphi_{A}<0, \quad \phi_{A}= \begin{cases}\phi_{0} & \text { se } \hat{\phi}_{A} \not[0,1] \\
10 \phi_{0}-9 \phi_{1} & \text { se } \hat{\phi}_{A} \in[0,3 / 74) \\
3 \phi_{1}+\frac{6}{8} \phi_{0}-\frac{1}{8} \phi_{1} & \text { se } \hat{\phi}_{1} \in[3 / 74,1 / 2) \\
1.5 \phi_{0}-0.5 \phi_{1} & \text { se } \hat{\phi}_{A} \in[1 / 2,2 / 3) \\
\phi_{-1} & \text { se } \hat{\phi}_{A} \in[2 / 3,1] .\end{cases}
\end{aligned}
$$

As expressões para $\hat{\phi}_{A}$ e $\hat{\phi}_{B}$ que aparecem nos esquemas HLPA e VONOS são definidas por

$$
\hat{\phi}_{A}=\left.\frac{\phi_{V}-\phi_{R}}{\phi_{D}-\phi_{R}}\right|_{P_{A}} \quad \text { e } \quad \hat{\phi}_{B}=\left.\frac{\phi_{l}-\phi_{R}}{\phi_{D}-\phi_{R}}\right|_{P_{A B}}
$$

em que $\phi_{U}$ valor da velocidado $\phi$ "upstream",$\phi_{R}$ valor da velocidade $\phi$ "remote-upstream" e $\phi_{D}$ valor da velocidade $\phi$ "downstream".

Neste trabalho utiliza-se o esquema VONOS que foi o esquema que se mostrou mais adequado para simulaçoos de escoamentos com superfícies livres (Ferreira et al., 2002); Souza, 2002). Para implementar os esefuemas acima considere por exemplo o termo $\frac{i z\left(u^{2}\right)}{2 x}$ 110 ponto $\left(i+\frac{1}{2}, j, k\right)$. Sua derivada é aproximada por

$$
\left.\frac{\partial\left(u^{2}\right)}{\partial x}\right|_{i+\frac{1}{2}, j, k}=\left.\frac{\partial(u u)}{\partial x}\right|_{t \rightarrow \frac{1}{2}, j, k} \approx \frac{\left(\hat{u}_{i+1, j, k} u_{i+1, j, k}-\hat{u}_{i, j, k} u_{i, j, k}\right)}{\delta x} .
$$

As velocidades de conveção na expressão acima são obtidas por uma média aritméticax. então

$$
\hat{u}_{i, j, k}=\frac{1}{2}\left(u_{i+\frac{1}{2}, j, k}+u_{i-\frac{1}{2}, j, k}\right) \quad e \quad \hat{u}_{i+1, j, k}=\frac{1}{2}\left(u_{i+\frac{3}{2}, j, k}+u_{i+\frac{1}{2}, j, k}\right) .
$$

Definindo 


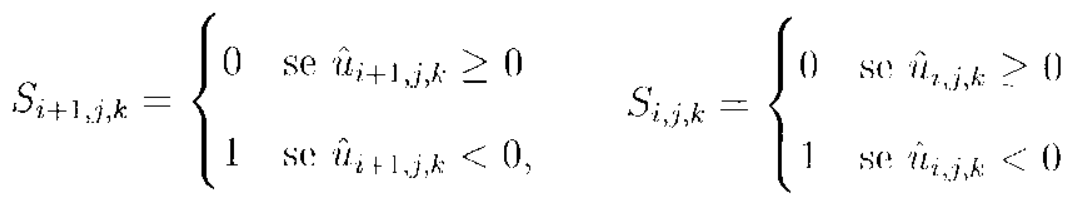

utilizando o esquema VONOS as velocidacles transportadias em (3.25) säo obtidas como

$$
\begin{aligned}
& \hat{\phi}_{i+\mathrm{T}, j, k}=\left(1-S_{i+1, j, k}\right)\left(\frac{u_{i+\frac{1}{2}, j, k}-u_{i-\frac{1}{2}, j, k}}{u_{i+\frac{3}{2}, j, k}-u_{i-\frac{1}{2}, j, k}}\right)+S_{i+1, j, k}\left(\frac{u_{i, \frac{3}{2}, j, k}-u_{i+\frac{5}{2}, j, k}}{u_{i+\frac{1}{2}, j, k}-u_{i+\frac{5}{2}, j, k}}\right)
\end{aligned}
$$

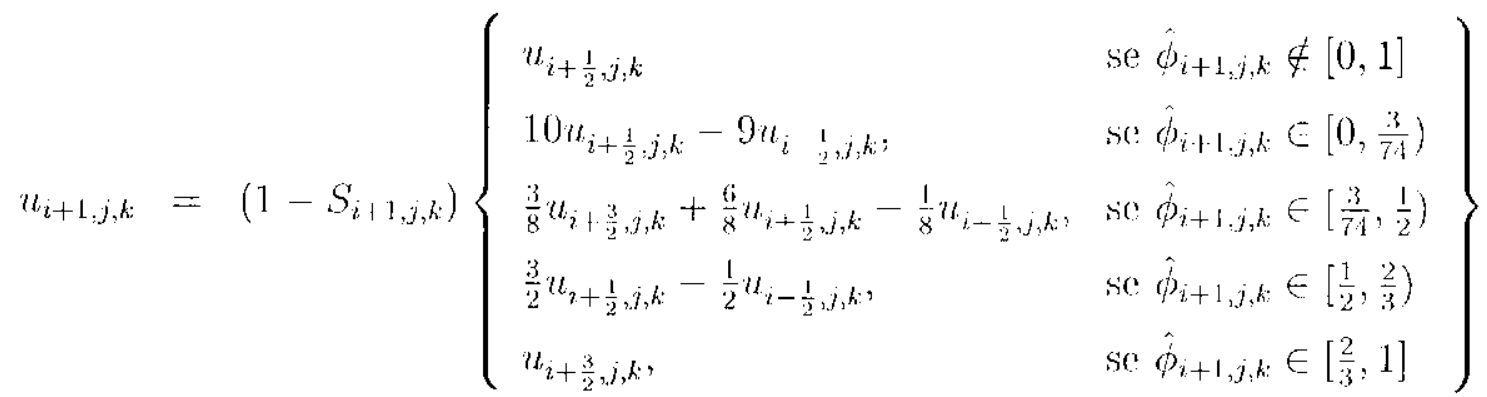

$$
\begin{aligned}
& +S_{i+1, j, k}\left\{\begin{array}{ll}
u_{i+\frac{3}{2}, j, k} & \text { se } \hat{\phi}_{i+1, j, k} \notin[0,1] \\
10 u_{i+\frac{3}{2}, j, k}-9 u_{i+\frac{5}{2}, j, k}: & \text { se } \hat{\phi}_{i+1, j, k} \in\left[0, \frac{3}{7.1}\right) \\
\frac{3}{8} u_{i+\frac{1}{2}, j, k}+\frac{6}{8} u_{i+\frac{3}{2}, j, k}-\frac{1}{8} u_{i+\frac{5}{2}, j, k}, & \text { se } \hat{\phi}_{i+1, j, k:} \in\left[\frac{3}{7.1}, \frac{1}{2}\right) \\
\frac{3}{2} u_{i+\frac{3}{2}, j, k}-\frac{1}{2} u_{i+\frac{5}{2}, j, k}, & \text { se } \hat{\phi}_{i-1, j, k} \in\left[\frac{1}{2}, \frac{2}{3}\right) \\
u_{i+\frac{1}{2}, j, k}, & \text { se } \hat{\phi}_{i+1, j, k} \in\left[\frac{2}{3}, 1\right]
\end{array}\right\} \\
& \hat{\phi}_{i, j, k}=\left(1-S_{i, j, k}\right)\left(\frac{u_{i-\frac{1}{2}, j, k}-u_{i-\frac{3}{2}, j, k}}{u_{i+\frac{1}{2}, j, k}-u_{i-\frac{3}{2}, j, k}}\right)+S_{i, j, k}\left(\frac{u_{i+\frac{1}{2}, j, k}-u_{i, \frac{3}{2}, j, k}}{u_{i-\frac{1}{2}, j, k}-u_{i+\frac{3}{2}, j, k}}\right) \\
& u_{i, j, k}=\left(1-S_{i, j, k}\right)\left\{\begin{array}{ll}
u_{i-\frac{1}{2}, j, k} & \text { se } \hat{\phi}_{i, j, k} \notin[0,1] \\
10 u_{i} \frac{1, j, k}{2}-9 u_{i} 3_{2, j, k}, & \text { se } \hat{\phi}_{i, j, k} \in\left[0, \frac{3}{74}\right) \\
\frac{3}{8} u_{i+\frac{1}{2}, j, k}+\frac{6}{8} u_{i-\frac{1}{2}, j, k}-\frac{1}{8} u_{i-\frac{3}{2}, j, k}, & \text { se } \hat{\phi}_{i, j, k} \in\left[\frac{3}{74}, \frac{1}{2}\right) \\
\frac{3}{2} u_{i-\frac{1}{2}, j, k}-\frac{1}{2} u_{i-\frac{3}{2}, j, k}, & \text { se } \hat{\phi}_{i, j, k} \in\left[\frac{1}{2}, \frac{2}{3}\right) \\
u_{i+\frac{1}{2}, j, k}, & \text { se } \hat{\phi}_{i, j, k} \in\left[\frac{2}{3}, 1\right]
\end{array}\right\} \\
& +S_{i, j, k}\left\{\begin{array}{ll}
u_{i+\frac{1}{2}, j, k} & \text { se } \hat{\phi}_{i, j, k} \notin[0,1] \\
10 u_{i+\frac{1}{2}, j, k}-9 u_{i+\frac{3}{2}, j, k}, & \text { se } \dot{\phi}_{i, j, k} \in\left[0, \frac{3}{74}\right) \\
\frac{3}{8} u_{i-\frac{1}{2}, j, k}+\frac{1}{8} u_{i+\frac{1}{2}, j, k}-\frac{1}{8} u_{i+\frac{3}{2}, j, k}, & \text { so } \hat{\phi}_{i, j, k} \in\left[\frac{3}{74}, \frac{1}{2}\right) \\
\frac{3}{2} u_{i+\frac{1}{2}, j, k}-\frac{1}{2} u_{i+\frac{3}{2}, j, k}, & \text { se } \hat{\phi}_{i, j, k} \in\left[\frac{1}{2}, \frac{2}{3}\right) \\
u_{i-\frac{1}{2}, j, k}, & \text { sc } \dot{\phi}_{i, j, k} \in\left[\frac{2}{3}, 1\right]
\end{array}\right\} .
\end{aligned}
$$


Os ontros termos convectivos são aproximados de mancira análoga a este procedimento.

\subsection{Discretização da Equação de Poisson para $\psi$}

Considere a equação de Poisson para o cálculo da fiunção potencial $\%$, dada em (2.10) por

$$
\nabla^{2} \psi(\mathbf{x}, t)=\nabla \cdot \tilde{\mathbf{u}}(\mathbf{x}, t)
$$

que em coordenadas cartesianas tridimensionais pode ser escrita como

$$
\frac{\partial^{2} \psi}{\partial x^{2}}+\frac{\partial^{2} \dot{\psi}}{\partial y^{2}}+\frac{\partial^{2} \psi}{\partial z^{2}}=\frac{\partial \tilde{u}}{\partial x}+\frac{\partial \tilde{y}}{\partial y}+\frac{\partial \tilde{w}}{\partial z}
$$

Aproximando (3.26) por diferenças centrais de segunda ordem na posiçăo $(i, j, k)$ temos

$$
\begin{aligned}
& \left.\frac{\partial^{2} \psi}{\partial x^{2}}\right|_{i, j, k} \approx \frac{\psi_{i+1, j, k}-2 \psi_{i, j, k}+u_{i-1, j, k}}{\delta x^{2}} \\
& \left.\frac{\partial^{2} \psi}{\partial y^{2}}\right|_{i, j, k} \approx \frac{\psi_{i, j+1, k}-2 \psi_{i, j, k}+u_{i, j-1, k}}{\delta y^{2}} \\
& \left.\frac{\partial^{2} \psi}{\partial z^{2}}\right|_{i, j, k} \approx \frac{\psi_{i, j, k+1}-2 \psi_{i, j, k}+u_{i, j, k-1}}{\delta z^{2}} \\
& \left.\frac{\partial^{2} \dot{\psi}}{\partial z^{2}}\right|_{i, j, k} \approx \frac{\psi_{i, j, k+1}-2 \psi_{i, j, k}+u_{i, j, k-1}}{\delta z^{2}} \\
& \left.\frac{\partial \tilde{u}}{\partial x}\right|_{i, j, k}-\frac{\tilde{u}_{i+\frac{1}{2}, j, k}-\tilde{u}_{i-\frac{1}{2}, j, k}}{\delta x} \\
& \left.\frac{\partial \tilde{v}}{\partial y}\right|_{i, j, k}=\frac{\tilde{v}_{i, j+\frac{1}{2}, k}-\tilde{v}_{i, j-\frac{1}{2}, k}}{\delta y} \\
& \left.\frac{\partial \tilde{w}}{\partial z}\right|_{i, j, k}=\frac{\tilde{u}_{i, j, k+\frac{1}{2}}-\tilde{u}_{i, j, k-\frac{1}{2}}}{\delta z} \text {. }
\end{aligned}
$$

Definindo

$$
\tilde{D}_{i, j, k}=\left.\frac{\partial \tilde{u}}{\partial x}\right|_{i, j, k}+\left.\frac{\partial \tilde{u}}{\partial y}\right|_{i, j, k}+\left.\frac{\partial \tilde{u}}{\partial z}\right|_{i, j, k}
$$

a discretização da equação (3.26) pode ser escrita como

$$
-\beta \psi_{i, j, k+1}-\alpha \psi_{i, j-1, k}-\psi_{i-1, j, k}+(2+2 \alpha+2 \beta) \psi_{i, j, k}-\psi_{i+1, j, k}-\alpha \psi_{i, j+1, k}-\beta \psi_{i, j, k+1}=\delta x^{2} \tilde{D}_{i, j, k}
$$


em que $\alpha=\frac{\delta x^{2}}{\partial y^{2}}$ e $\beta=\frac{\delta x^{2}}{\partial z^{2}}$.

A discretização de (3.26) resulta em um sistema de equaçós lineares que pode ser representiado por

$$
A \mathrm{x}=\mathrm{D}
$$

onde $A$ é uma matriz simétrica e definida positiva de ordem $n, \mathrm{x}$ co vetor solucäo de ordem $n$ e $\mathrm{D}$ o vetor divergente de ordem $n$, sendo $n$ o número de células (F) da malha. Para se resolver este sistema utiliza-se o método dos gradientes conjugarlos (Shewchuck, 1994) onde $\mathbf{x}$ ó inicializado como um vetor da função potencial no passo anterior.

\subsection{Condições de Contorno}

\subsubsection{Condições de Contorno na Superfície Livre}

Suponha que o espacamento da malha seja suficientemente pequeno para cuce a superfícic livre possa ser aproximada por uma superfície planar. Esta superfície planar pode ser paralela a um eixo coordenado, formar um ângulo de $45^{\circ} \mathrm{com}$ dois eixos coordenados ou um ângulo de $60^{\circ}$ com três cixos coordenados. Os três casos podem ser identificados por células de superfício (S) contendo uma, duas on três de suas faces adjacentes a urra célula vazia (E).

Por exemplo, se uma célula (S) tem somente a face $\left(k+\frac{1}{2}\right)$ adjacente a muna numal cólula (E), observe a figura 3.4 , centia neste caso tomat-se o vetor normal $\mathbf{n}=(0,0,1)$ e os vetores tangentes $\mathbf{m} \mathbf{1}=(1,0,0)$ e $\mathbf{m} 2=(0,1,0)$ e então as condiçoss $(1.34)$-(1.36) se reduzem a

$$
\begin{gathered}
p=\frac{2}{R e}\left(\frac{\partial w}{\partial z}\right) \\
\left(\frac{\partial v}{\partial z}+\frac{\partial w}{\partial y}\right)=0 \\
\left(\frac{\partial u}{\partial z}+\frac{\partial w}{\partial x}\right)=0
\end{gathered}
$$

As equaçoes (3.35) (3.37) juntamente com a equagăo de conscrvação de massa (1.27) são discretizadas resultando om um sistema algébrico que ć resolvido para encontrar as velocidades na célula (E) e a pressão no centro da cólula (S), ver figura 3.4. Outras configuracócs 


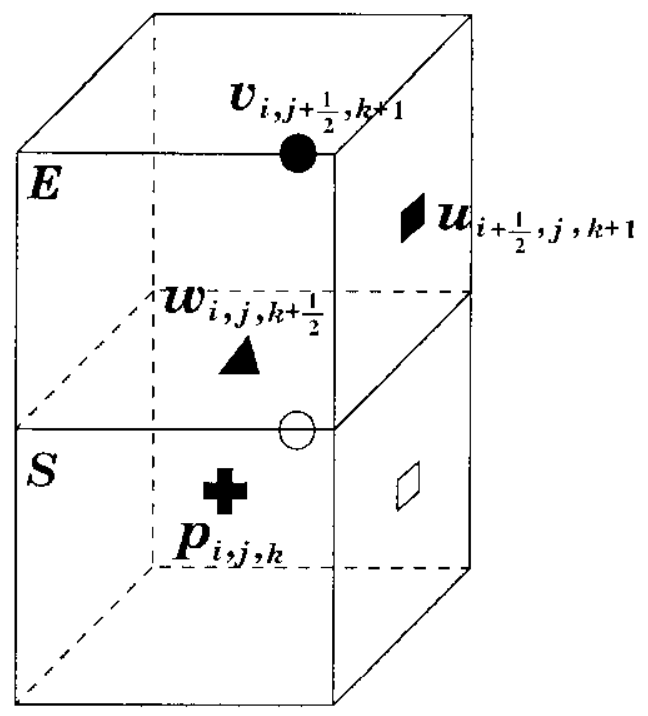

Figura 3.4: Cólula (S) com a face $\left(k+\frac{1}{2}\right)$ adjacente a célula (E).

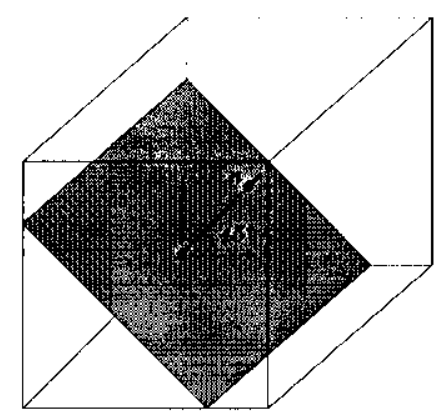

Figura 3.5: Exemplo de uma superfície planar inclinala a $45^{\prime \prime}$.

de células (S) tendo somente uma face adjacente a células (E) são tratadas analoganonte.

Para cólulas (S) tendo duas faces adjacentes a células (E) a superficíe planar forma um angulo de $45^{\prime \prime} \mathrm{com}$ os eixos das faces adjacentes, ver figura 3.5. Nessas superfícies assime-se que o velor nommal ó da forma $\mathbf{n}=\left( \pm \frac{\sqrt{2}}{2}, \pm \frac{\sqrt{2}}{2}, 0\right)$ ou $\mathbf{n}-\left(+\frac{\sqrt{2}}{2}, 0, \pm \frac{\sqrt{2}}{2}\right)$ ond $\mathbf{n}=\left(0, \pm \frac{\sqrt{2}}{2}, \pm \frac{\sqrt{2}}{2}\right)$. Por exemplo, considerando a cólula (S) mostrada na figura 3.6, tomase: $\mathbf{n}=\left(\frac{\sqrt{2}}{2}, 0, \frac{\sqrt{2}}{2}\right), \mathbf{m} \mathbf{1}=\left(\frac{\sqrt{2}}{2}, 0,-\frac{\sqrt{2}}{2}\right)$ e $\mathbf{m} 2=(0,1,0)$ entace as condiçes (1.34) $(1.36)$ se reduzem a

$$
\begin{gathered}
p=\frac{1}{R e}\left[\left(\frac{\partial u}{\partial x}+\frac{\partial w}{\partial z}\right)+\left(\frac{\partial u}{\partial z}+\frac{\partial u}{\partial x}\right)\right]=0 \\
\left(\frac{\partial u}{\partial x}-\frac{\partial w}{\partial z}\right)-0
\end{gathered}
$$




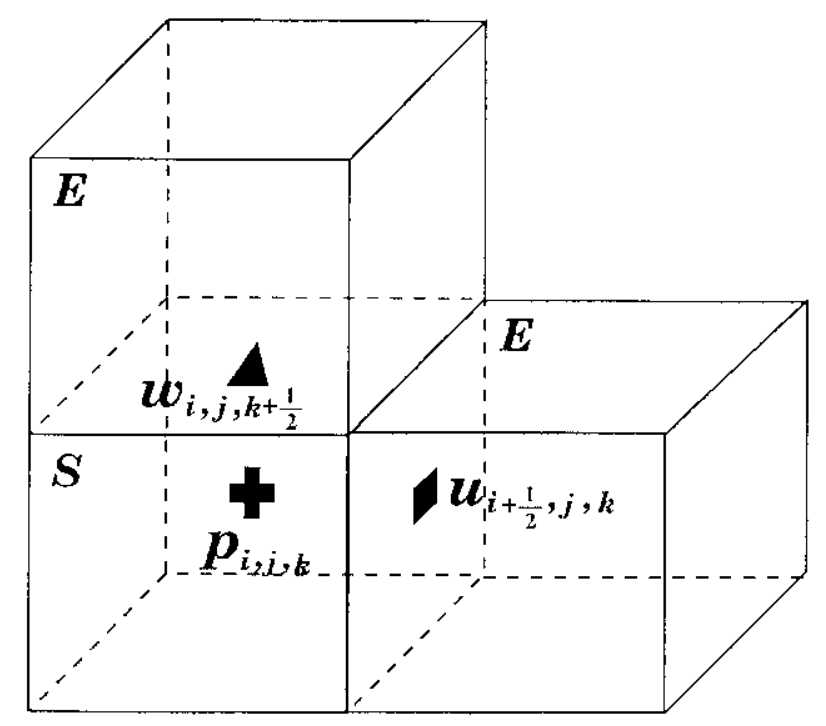

Figura 3.6: Cólula (S) com as faces $\left(k+\frac{1}{2}\right)$ e $\left(i+\frac{1}{2}\right)$ adjacentes a células (E).

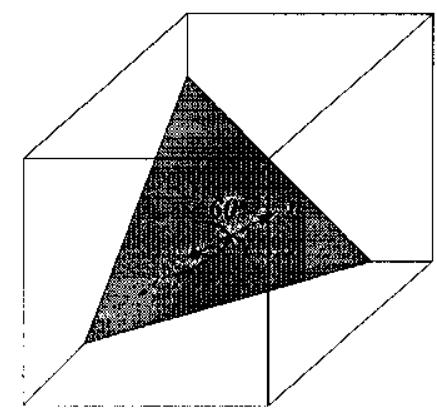

Figura 3.7: Fxemplo de uma superfície planar inclinada a $60^{\prime \prime}$.

A equação (3.38) é usada para calcular a pressäo no centro da célula (S) e as velocidades nas faces adjacentes às cólulas (E) são calculadas usando (3.39) juntamente com a equação de conservação de massa (1.27). Outras configuraçoes de cólulas (S) tendo duas faces arljacentes a células (E) são tratadas analogamente.

Para células (S) tendo três faces adjacentes a células (E) a superfície planar forma un angulo de $60^{n}$ com os três eixos coordenados, ver figura 3.7. Nestas superfícies assiunese que o vetor normal é da forma $\mathbf{n}=\left( \pm \frac{\sqrt{3}}{3}, \pm \frac{\sqrt{3}}{3}, \pm \frac{\sqrt{3}}{3}\right)$. Por exemplo, considerando a cólula (S) mostrada na figura 3.8, toma-se $\mathbf{n}=\left(\frac{\sqrt{3}}{3}, \frac{\sqrt{3}}{3}, \frac{\sqrt{3}}{3}\right), \mathbf{m} 1=\left(0, \frac{\sqrt{2}}{?},-\frac{\sqrt{2}}{2}\right)$ e $\mathrm{m} 2=\left(-2 \frac{\sqrt{6}}{6}, \frac{\sqrt{6}}{6}, \frac{\sqrt{6}}{6}\right)$ e então as condições $(1.34)-(1.36)$ se reduzem a

$$
p-\frac{2}{3 R e}\left[\left(\frac{\partial u}{\partial y}+\frac{\partial v}{\partial x}\right)+\left(\frac{\partial u}{\partial z}+\frac{\partial w}{\partial x}\right)+\left(\frac{\partial u}{\partial z}+\frac{\partial w}{\partial y}\right)\right]=0
$$




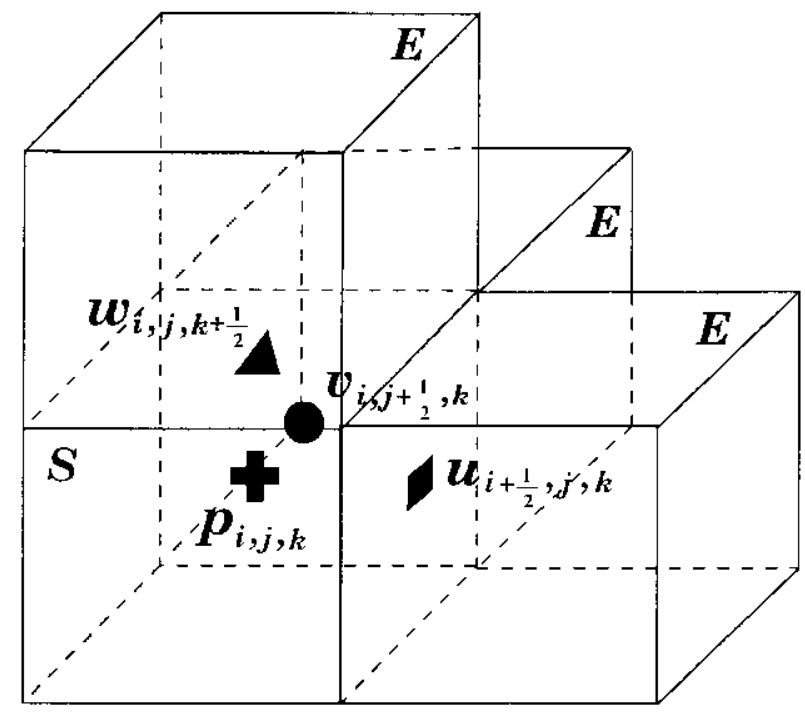

Figura 3.8: Célula (S) com as faces $\left(i+\frac{1}{2}\right),\left(k+\frac{1}{2}\right)$ e $\left(j+\frac{1}{2}\right)$ adjacentes a células (E).

$$
\begin{gathered}
2 \frac{\partial v}{\partial y}-2 \frac{\partial u}{\partial z}+\left(\frac{\partial u}{\partial y}+\frac{\partial v}{\partial x}\right)-\left(\frac{\partial u}{\partial z}+\frac{\partial w}{\partial x}\right)=0 \\
-4 \frac{\partial u}{\partial x}+2 \frac{\partial v}{\partial y}+2 \frac{\partial w}{\partial z}-\left(\frac{\partial u}{\partial y}+\frac{\partial v}{\partial x}\right)-2\left(\frac{\partial u}{\partial z}+\frac{\partial w}{\partial x}\right)+2\left(\frac{\partial v}{\partial z}+\frac{\partial w}{\partial y}\right)=0
\end{gathered}
$$

As equaçōes (3.40) (3.42) juntamente com a equação de conservaçăo de massa (1.27) são usadas para se obter a pressão no centro da célula (S) e as velocidades nas fares adjacentes às cólulas (F). Outras configurações de células (S) tendo três faces adjacentes as cólulas (E) são tratadas analogamente.

Ainda há casos de cólulas (S) tendo dois lados opostos adjacentes a células (E). Um tratamento para esses casos consiste em definir a pressão mula no centro da cólula (S) a as velocidades são calculadas de modo a satisfazer a equaça de conservação do massa. Una descrição detalhada de cada caso pode ser encontrada en (Tomé et al., 1996a).

Para o cálculo da temperatura na superfície livre foi utilizado o seguinte procedimento. Considere uma cólula de superfície $S_{i, j, k}$ e suponha cue o balanço de calor sobre as faces dessa célula adjacentes a células (E) seja nulo. Scrão considerados os casos em que a célula (S) contém uma, duas, três, quatro, cinco ou seis de suas faces adjacentes a umal cólula (E). Lembrando que a condição de contorno da temperatura na superfície livie é dada pela equação (1.37) e integrando-se sobre a superfície da célula (S) sobre as faces 


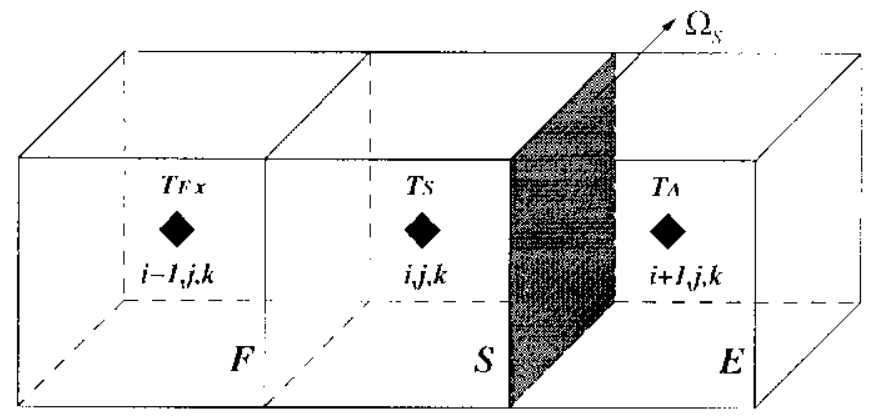

Figura 3.9: Cólula (S) tendo uma face adjacente a uma cólula (E).

adjacentes as células (E) obtemos

$$
\int_{\Omega_{S}}-\nabla T \cdot \mathbf{n} d S=\int_{\Omega_{S}} \operatorname{Nu}\left(T_{S} \cdots T_{2}\right) d S
$$

em que $\Omega_{S}$ denota a superlícic livre associada à cólula $(S)$.

Agora considera-se alguns casos de modo que possamos obter uma fómmula geral para calcularmos a temperatura na superfície livre. Considerando que a cólula (S) possui somente. uma cólula adjacente (E), como na figura 3.9 e scja $T_{i, j, k}^{\prime}=T_{S}^{\prime}$ o valor da temperatura a ser obtido na célula (S), $T_{F}$ o valor da temperatura na célıla (F) oposta a célula (E) e $T_{A}$ o valor de referência para a temperatura no ambiente, então (3.43) porle ser aproximada por

$$
-\frac{T_{S}-T_{F I}}{\delta x} \delta y \delta z-\operatorname{Nu}\left(T_{S}-T_{A}\right) \delta y \delta z
$$

$\log O$

$$
T_{S}=\frac{T_{l x}+\delta x \mathrm{Nu} T_{A}}{1+\delta x \mathrm{Nu}}
$$

em que $T_{r x}$ é a temperatura na célula $(\mathrm{F})$ na direção $x$.

Considerando agora que a célula (S) possui duas cólulas adjaccntes (L). como na figura 3.10 , entào (3.43) pode ser aproximada por

$$
-\left(\frac{T_{S}-T_{F^{\prime} x}}{\delta x} \delta y \delta z+\frac{T_{S}^{\prime}-T_{F^{\prime} y}}{\delta y} \delta x \delta z\right)=\mathrm{Nu}\left(T_{S}-T_{A}\right) \delta y \delta z+\mathrm{N}_{11}\left(T_{S}-T_{A}\right) \delta x \delta z
$$

$\log 0$

$$
T_{S}=\frac{T_{F x} \frac{\delta y \delta z}{\delta x}+T_{r y y} \frac{\delta x \delta z}{\delta y}+N_{11} T_{A}(\delta y \delta z+\delta x \delta z)}{\frac{\delta y \delta z}{\delta x}+\frac{\delta x \delta z}{\delta y}+\operatorname{Nu}(\delta y \delta z+\delta x \delta z)}
$$




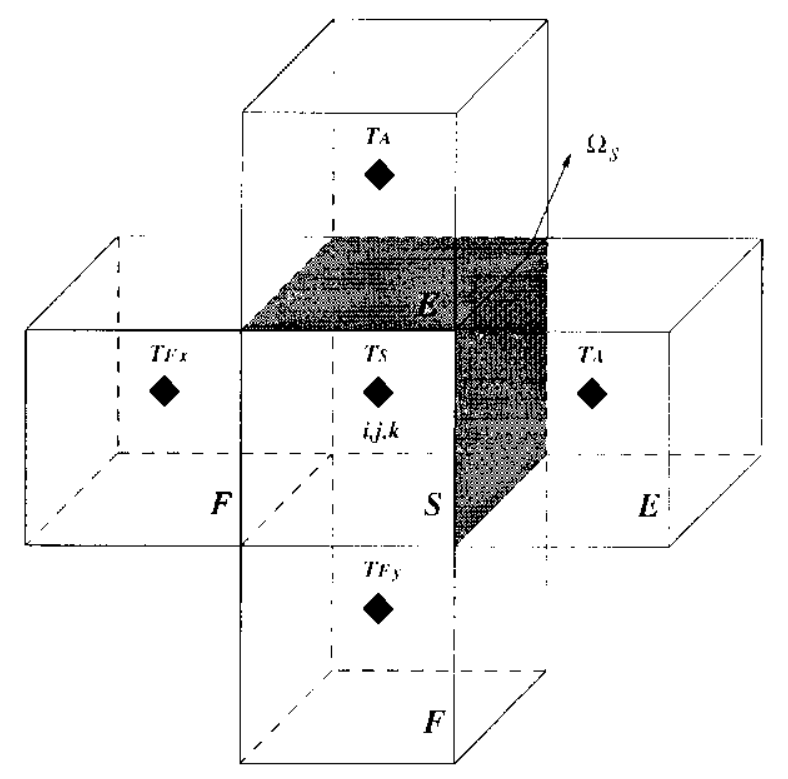

Figura 3.10: Célula (S) tendo duas faces adjacentes a cólulas (E).

Considerando que $\delta x=\delta y=\delta z=\delta$ a equaçầ (3.17) torma-se

$$
T_{S}=\frac{T_{F x}+T_{F y}+2 \delta \mathrm{Nu} T_{A}}{2+2 \delta \mathrm{Nu}}
$$

en que $T_{F x}$ é a temperatura na célula (F) oposta a célula (E) na direção $x$ e $T_{F y}$ é a temperatura na célula (F) oposta a célula (E) na diregão y. Ontros casos poderäo ser obtidos de mancira análoga.

Notando o desenvolvinento das equaçoes para o cálculo da temperatura na superfície livre nos casos acima o sendo $\delta x=\delta y=\delta z=\delta$ podemos entäo usar a seguinte fórmula geral para calcularmos a temperatura na superfície live

$$
T_{S}=\frac{\sum T_{F^{2}}+\delta \mathrm{Nu} T_{A} \# C_{E}}{\# C_{F}+\delta \mathrm{N} u \# C_{L}}
$$

rm que $\sum T_{F}$ é a soma das temperaturas das células (F) adjacentes as faces da célula (S) e opostas a células (E), \#Co é o múmero das células (E) adjacento as faces da cólula (S) e \#CF é o número das cólulas (F) adjacente as faces da célula (S) o opostas as cólulas (E). O fato de considerarmos que $\delta x=\delta y=\delta z=\delta$ não impôe uma restriçẫo adicional ao código, visto que a aproximação das condições de contorno na superfície livre exigem que $\delta x=\delta y=\delta z$. 


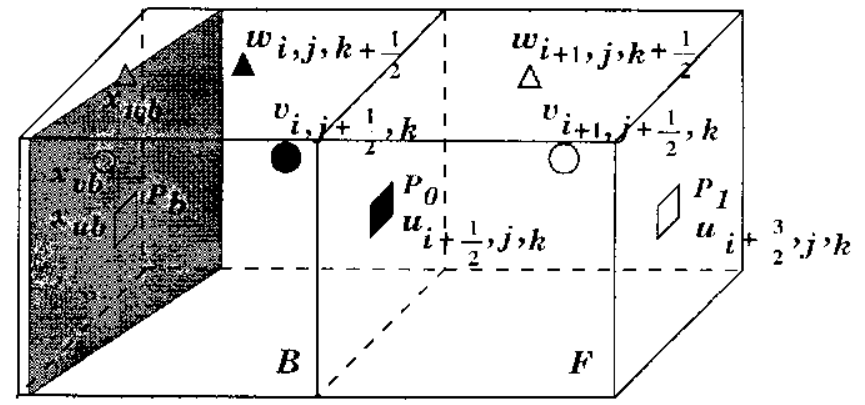

Figura 3.11: Cólula (B) com a face $\left(i+\frac{1}{2}\right)$ adjacente com uma cólula $(S)$ ou $(F)$.

\subsubsection{Condições no Contorno Rígido}

O método utilizado pra o cálculo das velocidades $u, v$ e $w$ nas faces das cólulas do frontcira é a interpolação linear. Essas células podem ter um, dois ou três lados adjacentes as células de superfícic on as célulàs cheias. Tratando de problemas tridimensionais tền-se 26 casos possíveis ('Tomé et al., 1996a). Como excmplo de aplicação do método consideram-so alguns exemplos.

Considere o caso em que uma cólula (B) possui a face $\left(i \quad \frac{1}{2}\right)$ em contato com uma célula (F) on (S), como na figura 3.11.

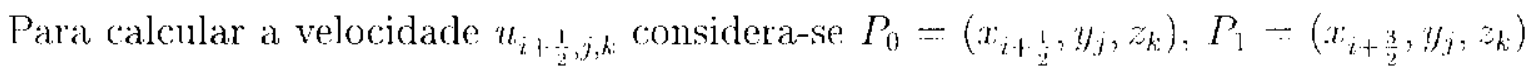
e $P_{b}=\left(x_{u b}, y_{j}, z_{k}\right)$, em que $x_{u b}$ representa a intersecção entre a reta definida por $P_{0}$ e $P_{1}$ e a superfície da fronteira. O valor de $x_{u b}$ ć dado pela interseção dos planos $y=y_{j}, z=z_{k}$. com a fronteira local. Portanto a interpolação linear entre $P_{b}$ e $P_{1}$ é

$$
u(x)=\frac{x-x_{i+\frac{3}{2}}}{x_{u b}-x_{i+\frac{3}{2}}} u_{b}+\frac{x-x_{u_{b}}}{x_{i+\frac{3}{3}}-x_{u_{b}}} u_{i+\frac{3}{2}, j, k}
$$

assim $u_{i+\frac{1}{2}, j, k}$ pode ser calculado por

$$
u_{i+\frac{1}{2}, j, k}=\frac{x_{i+\frac{1}{2}} \cdots x_{u b}}{x_{i+\frac{3}{2}}-x_{u b}} u_{i+\frac{3}{2}, j, k}-\frac{\delta x}{x_{u b} \cdots x_{i+\frac{3}{2}}} u_{b}
$$

cm que $u_{b}$ é a velocidade da fronteira na direção) $x\left(u_{b}=0\right.$ se a condição "no-slip" é aplicada sobre esta fronteira). As outras duas velocidades sobre as faces da célula de fronteira são obtidas de modo análogo e são dadas por 


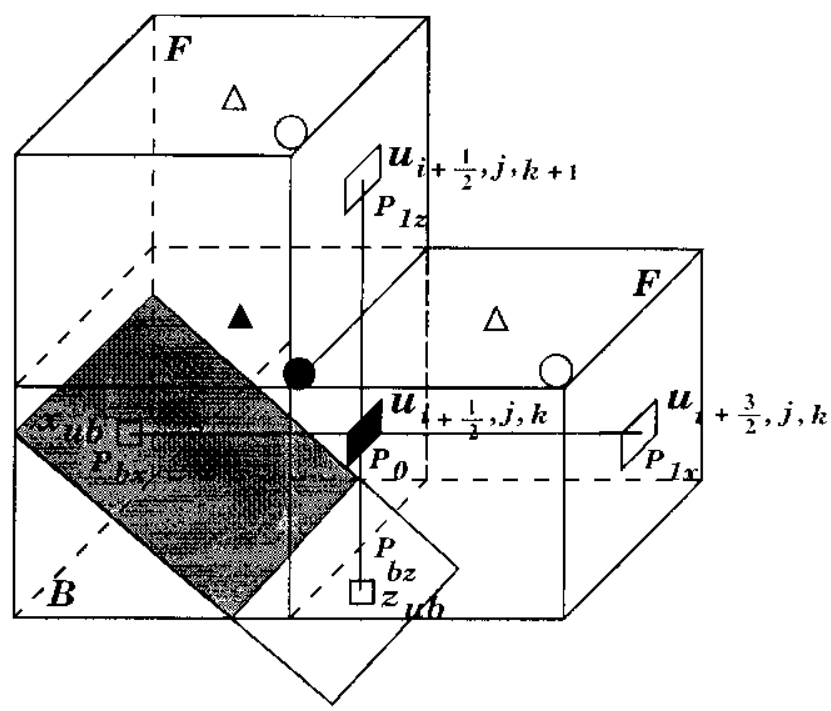

Figura 3.12: Célula (B) com as laces $\left(i+\frac{1}{2}\right)$ e $\left(j+\frac{1}{2}\right)$ adjacentes a células (S) ou (F).

$$
\begin{gathered}
v_{i, j+\frac{1}{2}, k}=\frac{x_{i}-x_{v b}}{x_{i+1}-x_{w h}} v_{i+1, j+\frac{1}{2}, k}-\frac{\delta x}{x_{y b}-x_{i+1}} v_{b} \\
w_{i, j, k+\frac{1}{2}}-\frac{x_{i}-x_{w b}}{x_{i+1} \cdots x_{w b}} w_{i+1, j, k+\frac{1}{2}}-\frac{\delta x}{x_{u, b} \cdot x_{i+1}} w_{b}
\end{gathered}
$$

em que $x_{v b}$ e $x_{u: b}$ são dados pela intersecção dos planos $y=y_{j-\frac{1}{2}}, z=z_{k}$ cont a fronteira local o $y=y_{j}, z=z_{k+\frac{1}{2}}$ com a fronteira local respectivamente e $v_{b}$ a $w_{b}$ säo as velocidades da fronteira ras direçoes $y$ e $z$ respectivamente. Outras configuracões de células de fronteira tendo uma face adjacente em contato com uma cólula (S) ou (F) são tratadas de modo semelhante.

Considere o caso que a célula (B) com as faces $\left(i+\frac{1}{2}\right)$ e $\left(j+\frac{1}{2}\right)$ está em contato com células (S) ou (F), como mostra a figura 3.12.

Calcula-se a velocidade $u_{i+\frac{1}{2}, j, k}$ por interpolação lincar que pode ser feita na direção $x$ 11sando $u_{i+\frac{3}{2}, j, k}$ e $u_{b}$ ou na direção $z$ usando $u_{i+\frac{1}{2}, j, k+1}$ e $u_{b}$. Para fazer a interpolação devese determinar qual direção é mais apropriada como será descrito a seguir. Considerase $P_{0}=\left(x_{i+\frac{1}{2}}, y_{j}, z_{k}\right), P_{1 x}=\left(x_{i+\frac{3}{2}}, y_{j}, z_{k}\right), P_{b x}=\left(x_{u b}, y_{j}, z_{k}\right), P_{1 z}-\left(x_{i+\frac{1}{2}}, y_{j}, z_{k+1}\right)$ e $P_{b z}-\left(x_{i+\frac{1}{2}}, y_{j}, z_{u b}\right)$, em que $x_{u b}$ representa a intersecgio entre a reta definida por $P_{0}$ e. $P_{1 x}$ e a superfície da fronteira e $z_{u b}$ representa a intersecgão entre a reta definida por $P_{0}$ e $P_{1 z}$ e a superfície da fronteira. Os valores de $x_{n b}$ e $z_{n b}$ são dados pela interseccão dos planos $y=y_{j}, z=z_{k}$ com a fronteira local $\mathrm{x} x=x_{i+\frac{1}{2}}, y=y_{j}$ com a fronteira local 
respectivamente. Com $x_{u b}$ e $\hat{z}_{u b}$ obtidos nós calculamos as distáncias

$$
d_{x u}=\left|x_{u b}-x_{i+\frac{1}{2}}\right| \quad \text { e } \quad d_{z u}=\left|z_{u t}-z_{k}\right|
$$

Para escolher a direção para interpolarmos toma-se o ponto mais próximo de $P_{0}$. Por exemplo so $d_{x u}<d_{z u}$ toma-se $P_{b x}$ e interpola-se entre $P_{b x \cdot r}$ e $P_{1 x}$. Neste caso $u_{i+\frac{1}{2}, j, k}$ a dada por

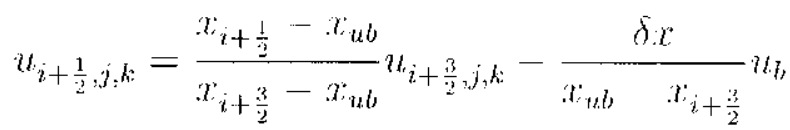

Mas se $d_{n u}>d_{z u}$ entäo toma-se $P_{b z}$ e interpola-se entre $P_{b z}$ \& $P_{1 z}$. Neste (also $u_{i+\frac{1}{2}, \xi, k} 6$ dada por

$$
u_{i+\frac{1}{2}, j, k}=\frac{z_{k}-z_{u b}}{z_{k+1}-z_{u b}} u_{i+\frac{1}{2}, j, k+1}-\frac{d z}{z_{u b}-z_{k+1}} u_{b} .
$$

As outras duas velocidades $v_{i, j+\frac{1}{2}, k} \% w_{i, j, k+\frac{1}{2}}$ sầ obtidas analogamente aplicando o mesmo critério. Outras configurações de células de fronteira tendo duas faces adjacentes em contato com células (S) ou (F) são tratadas de modo semelhante.

Considere o caso que a célula (B) com as faces $\left(i+\frac{1}{2}\right),\left(j+\frac{1}{2}\right)$ e $\left(k+\frac{1}{2}\right)$ está erm contato com células (S) ou (F), como mostra a figura 3.13.

Para o cálculo da velocidacle $u_{i+\frac{1}{2}, j, k}$ a interpolação linear pode ser feita na direção $x$

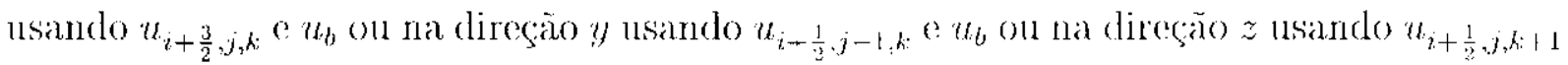
c $u_{b}$. Para fazer a interpolagão adota-se o mesmo critério no aso de termos configuracoses de células de fronteira tendo duas faces adjacentes em contiato com cólulas (S) on (F) visto anteriormente. Considera-se $P_{0}=\left(x_{i+\frac{1}{2}}, y_{j}, z_{k}\right) . P_{1: x}=\left(x_{i+\frac{3}{2}}, y_{j}, z_{k}\right), P_{b x}=\left(x_{u b}, y_{j}, z_{k}\right)$, $P_{1 y}=\left(x_{i+\frac{1}{2}}, y_{j-1}, z_{k}\right), P_{b y}=\left(x_{i+\frac{1}{2}}, y_{u b}, z_{k}\right), P_{1 z}=\left(x_{i 1}, y_{j}, z_{k+1}\right) \odot P_{b z}=\left(x_{i+\frac{1}{2}}, y_{j}, z_{u b}\right)$, em que $x_{u b}$ representa a interseção entre a reta definida por $P_{0} \subset P_{1 . r}$ e a superfícice da fronteira, em que $x_{y b}$ representa a intersecção cntre a reta definida por $P_{0}$ e $P_{1 y}$ e a superfície da fronteira e $z_{u b}$ representa a intersecção entre a reta definicla por $P_{0}$ e $P_{1 z}$ e al superfície da fronteira. O valores de $x_{u b}, y_{u b}$ e $z_{u b}$ são dados pela interseção dos planos $y=y_{j}, z=z_{k}$ com a frontcira local, $x=x_{i+\frac{1}{2}}, z=z_{k}$ com a fronteira local e $x=x_{i+\frac{1}{2}}$, $y=y_{j}$ com a fronteira local respectivamente. Com $x_{u b}, y_{u b}$ : $z_{u b}$ obtidos calculam-se as 


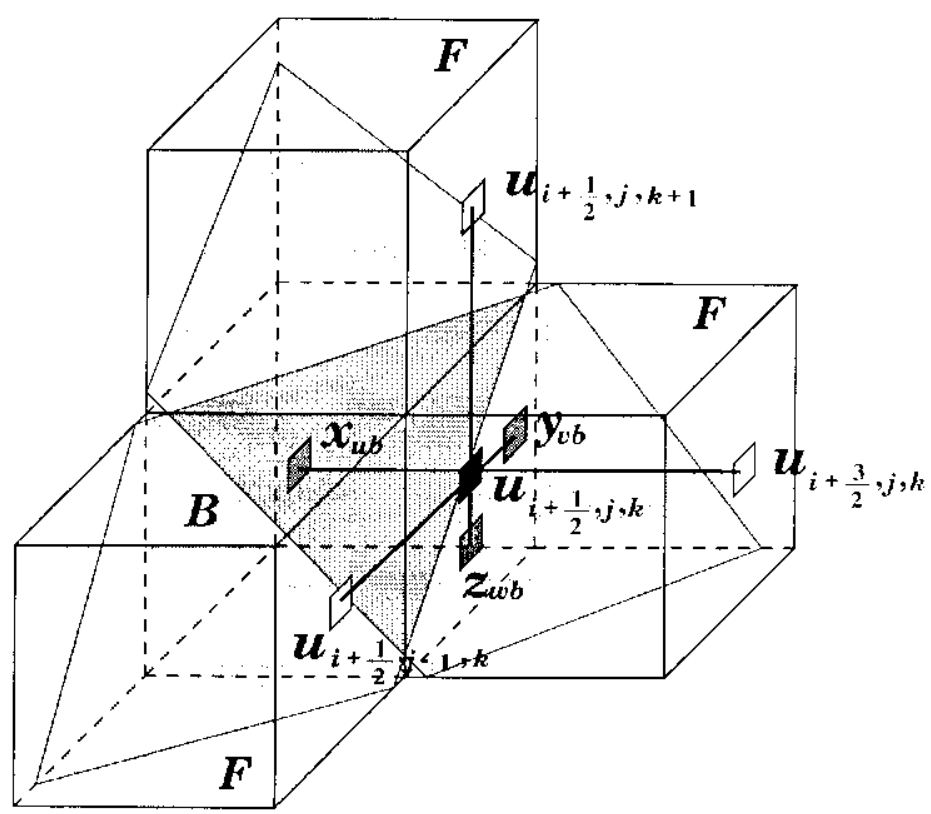

Figura 3.13: Célula (B) com as faces $\left(i+\frac{1}{2}\right),\left(j+\frac{1}{2}\right)$ e $\left(k+\frac{1}{2}\right)$ adjaccntes a células (S) ou (F).

distâncias

$$
d_{x u}=\left|x_{u b}-x_{i+\frac{1}{2}}\right|, \quad d_{y u}=\left|y_{u b}-y_{j}\right| \quad \text { e } \quad d_{z u}=\left|z_{u b}-z_{k}\right|
$$

Para cscolher a direção para interpolarmos toma-se o ponto mais próximo lo $P_{0}$, isto ć, seja

$$
d_{\min }=\min \left\{d_{x u}, d_{y_{u}}, d_{z u}\right\}
$$

Por cxemplo se $d_{m i n}=d_{x n}$ interpola-se entre $P_{b x x}$ e $P_{1 x x}$. Neste caso $u_{i+t_{n}, j, k}$ é dada por

$$
u_{i+\frac{1}{2}, j, k}=\frac{x_{i+\frac{1}{2}}-x_{u b}}{x_{i+\frac{3}{2}}-x_{u b}} u_{i+\frac{3}{2}, j, k}-\frac{\delta x}{x_{u b}-x_{i+\frac{3}{2}}} u_{b} .
$$

Mas se $d_{\min }=d_{y y}$ então interpola-se entre $P_{b y}$ e $P_{1 z}$. Neste caso $u_{i: \frac{1}{2}, j, k}$ é dada por

$$
u_{i+\frac{1}{2}, j, k}=\frac{y_{j}-y_{u b}}{y_{j-1}-y_{u b}} u_{i+\frac{1}{2}, j-1, k}-\frac{\delta y}{y_{n u}-y_{j+1}} u_{b}
$$

Mas se $d_{m i n}=d_{z u}$ então interpola-se entre $P_{b z}$ e $P_{1 z}$. Neste caso $u_{i+\frac{1}{2}, j, k}$ é dada por

$$
u_{i+\frac{1}{i}, j, k}=\frac{z_{k}-z_{u b}}{z_{k+1}-z_{u b}} u_{i+\frac{1}{2}, j, k+1}-\frac{\delta z}{z_{u b}-z_{k+1}} u_{b}
$$




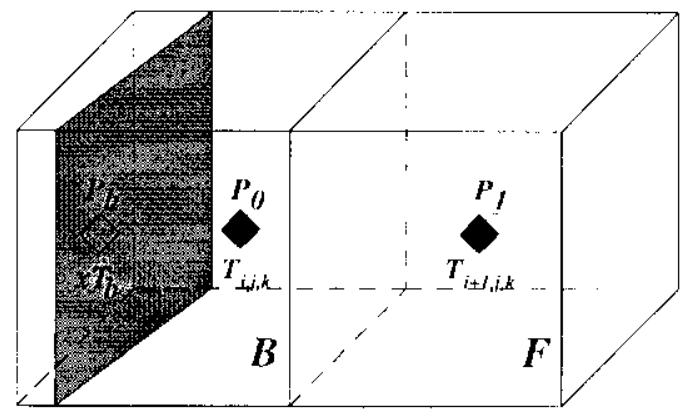

Figura 3.14: Célula (B) com a face $\left(i+\frac{1}{2}\right)$ adjacente a célula (S) ou (F).

As outras duas velocidades $v_{i, j+\frac{1}{2}, k}$ e $w_{i, j, k-\frac{1}{2}}$ são obtidas analogamente aplicanclo o mesmo critério. Outras configuracooes de células de fronteira tendo trîs fáces adjacentes em contato com células (S) ou (F) são tratadas de modo semelhante. Detalles de cadal (aso pode ser visto em ('Tomé et al., 1996a).

O procedimento para o cáleulo da temperatura nas células de fronteira ć semelhante ao que foi feito para as velocidades sendo que a temperatuma ć calculada no centro das células de fronteira. Como exemplo considera-se o caso em que temos uma célula (B) com a face $\left(i+\frac{1}{2}\right)$ em contato com uma célula $(S)$ ou $(F)$ como na figura 3.14 .

Para o cálculo da temperatura $T_{i, j, k}$ considera-se $\Gamma_{0}=\left(x_{i}, y_{j}, z_{k}\right), P_{1}=\left(x_{i+1}, y_{j}, z_{k}\right)$ e $P_{b}=\left(x_{T b}, y_{j}, z_{k}\right)$, em que $x_{T b}$ representa a intersecçấ entre a reta definida por $P_{0}$ o: $P_{1}$ e a superfície da fronteira. O valor de $x_{T b}$ é dado pela intersecça dos planos $y=y_{j}: z=z_{k}$ com a fronteira local. Portanto o valor de $T_{i, j, k}$ dado pela interpolação lincar entre $P_{b} \mathrm{c}$ $P_{1}$ ¿́

$$
T_{i, j, k}-\frac{x_{i}-x_{T b}}{x_{i+1}-x_{T b}} T_{i+1, j, k}-\frac{\delta x}{x_{T b}-x_{i+1}} T_{b}
$$

em que $T_{b}$ é a temperatura da fronteira na diregão $x$. Outras configuraçoses de cólulas (B) tendo uma, duas on tres faces adjacentes em contato com células (S) on (F) säo tratadas de forma semellante.

Quando utilizada a condição de fronteira adiábatica a temperatura nas células de fronteira (B) é calculada usando o seguinte procedimento. Seja $\Lambda$ o conjunto dos írdices das células adjacentes às laces da célula $(i, j, k)$ que săo classificadas como $(F)$ ou $(S)$ o $n$ o número de elementos em $\Lambda$. A temperatura na célula de fronteira (B) $T_{i, j, k}$ é dada por 


$$
T_{i, j, k}-\frac{1}{n} \sum_{\lambda \in \Lambda} T_{\lambda}
$$

\subsection{Controle do Passo no Tempo}

() tamanho do passo no tempo é obtido sujeito as scguintes restriçoces de estabilidade:

- nenhuma partícula cruzará mais de uma célula em um dado intervalo de tempo, isto é:

$$
\delta t<\frac{\delta x}{|u|}, \quad \delta t<\frac{\delta y}{|v|} \quad \text { e } \quad \delta t<\frac{\delta z}{|w|}
$$

sendo suficiente cue

$$
\delta t<\frac{\delta x}{\left|u_{\text {max }}\right|}, \quad \delta t<\frac{\delta y}{\left|v_{\text {max }}\right|}, \quad \text { e } \quad \delta t<\frac{\delta z}{\left|w_{\text {max }}\right|}
$$

em que $u_{\text {max }}, v_{\text {max }}$ e $w_{\text {max }}$ são os valores máximos de $u, v$ e $u$.

- devido a discretização explícila das equações de conservação de quantidate de movimento adota-se a restrição de estabilidade (Tomé et al., 1996b)

$$
\delta t<\frac{\operatorname{lie}}{2 \nu(T)} \frac{\delta x^{2} \delta y^{2} \delta z^{2}}{\delta x^{2}+\delta y^{2}+\delta z^{2}}
$$

sendo suficiente que

$$
\delta t<\frac{\operatorname{Re}}{2 \nu_{\max }} \frac{\delta x^{2} \delta y^{2} \delta z^{2}}{\delta x^{2}+\delta y^{2}+\delta z^{2}}
$$

- a equação da energia requer uma restrição de estabilidade que complementa as restrições acima e á análoga ao item anterior (Griebel et al., 1997)

$$
\delta t<\frac{\operatorname{RoPr}}{2} \frac{\delta x^{2} \delta y^{2} \delta z^{2}}{\delta x^{2}+\delta y^{2}+\delta z^{2}}
$$




\section{Capítulo 4}

\section{O Ambiente de Simulação \\ FreeFlow-3D para Escoamentos Não-Isotérmicos}

Este capítulo apresenta de forma resumida o sistema FreeFlow-3D) (Castelo Filho ct al., 1999), a as modilicaçós feitas em seus módulos para que o mesmo possá simular escoamentos não-isotírmicos.

\subsection{O Ambiente de Simulação FreeFlow-3D}

O ambiente de simulação tridimensional para escoamentos incompressíveis com superfícies livres é constituído por quatro módulos, os quais săo:

- Modflow-3D (modelador de moldes e escoamentos): tratia-se de um módulo interativo para a especificação inicial de dados que caracterizam o escoamento a ser simulado e possibilita que elementos no domínio do escoanento sejam definidos. Este módulo inclui elementos tais como recipientes, injetores, e fluidos;

- Simflow-3D (simulador de escoamentos): este módulo é a parte central do FreeFlow3D onde são resolvidas as equações govemantes do escoamento juntamente com as condições de contorno;

- Visflow-31) (visualizador de escoamentos): este módulo interativo permitr an usurírio 
visualizar a saída do Simflow-3Г) en imagens nos tempos de impressia pré-estabelecidos no modelador:

- Resimflow-3D (reiniciador de escoamentos): este módulo permite ao usuário reiniciar a simmlação do ponto em que foi interrompida.

A implementação dos quatro módulos loi feita em linguagem de programaçăo C sobre o sistema operacional Unix. A commicação dos módulos do sistemá é efetuada através de arquivos de dados.

\subsection{Estrutura de Dados}

Os dados no sistema FreeFlow-3D estão divididos em duas classes: dados diretos o indiretos (Paiva, 2000). O conjunto de diddos diretos contêm darlos referentes ao domínio, velocidade, pressão, células, parâmetros usados pelo simulador c a representaçào dos objetos geométricos do modelo. Este dados estão subdividiclos ems:

- dados estáticos (não são modificados durante a simulação): definiçã̃o do domínio, espaçamento da malha, parâmetros de adimensionalização entre outros;

- dados dinâmicos (que se modificam durante a simulação): campo do volocirlades, pressão, configuração dos tipos de células, posiçào da superfície livere entre ontros.

Os dados indiretos são representados por três estruturas:

- recipientes: representam os recipientes e é composta por dados geométricos, tipos de condições de contorno, as células que o definem e atributos específicos do recipiente representado;

- injetor: representa os injetores e é composta por dados geométricos, informacouss sobre o recipiente e ofluido que relacionarn-so com esse injetor, as cólulas que o definem e atributos específicos;

- ejotor: representam os ejetores e e composta por darkes geométricos, tipos de condições de contorno, informaçoes sobre o recipiente que o contém, as cólulas que o definem e atributos específicos. 
Dados como velocidades a pressiu săo representados por matrizes, assim como os identificadores das células (F, S, I, O e B), que são armazenados em uma matriz chamada "CELL", os quais são atualizados em cada passo no tempo, enquanto que cada grupo de. células ( $\mathrm{F}, \mathrm{S}, \mathrm{I}, \mathrm{O}$ e B) é representado por uma estrutura de dados do tipo árvore, contendo informações e configuraçōes destas células. Os objetos geométricos são representiados por uma estrutura de dados do tipo B-Rep ("Boundary Representation"), que armazcra objetos pela sua fronteira (sólidos, faces, arestas, vértices e sulas estruturas de incidência (adjacêcia). A estrutura de dados utilizada é denominada "half-edge" e esta dividida em seis níveis hierárquicos. Cada nível da estrutura "half-edge" esta representado na figura 4.1. Cada nível é representado por listas duplamente encadeadas, sendo que a lista de: semi-arestas é circular.

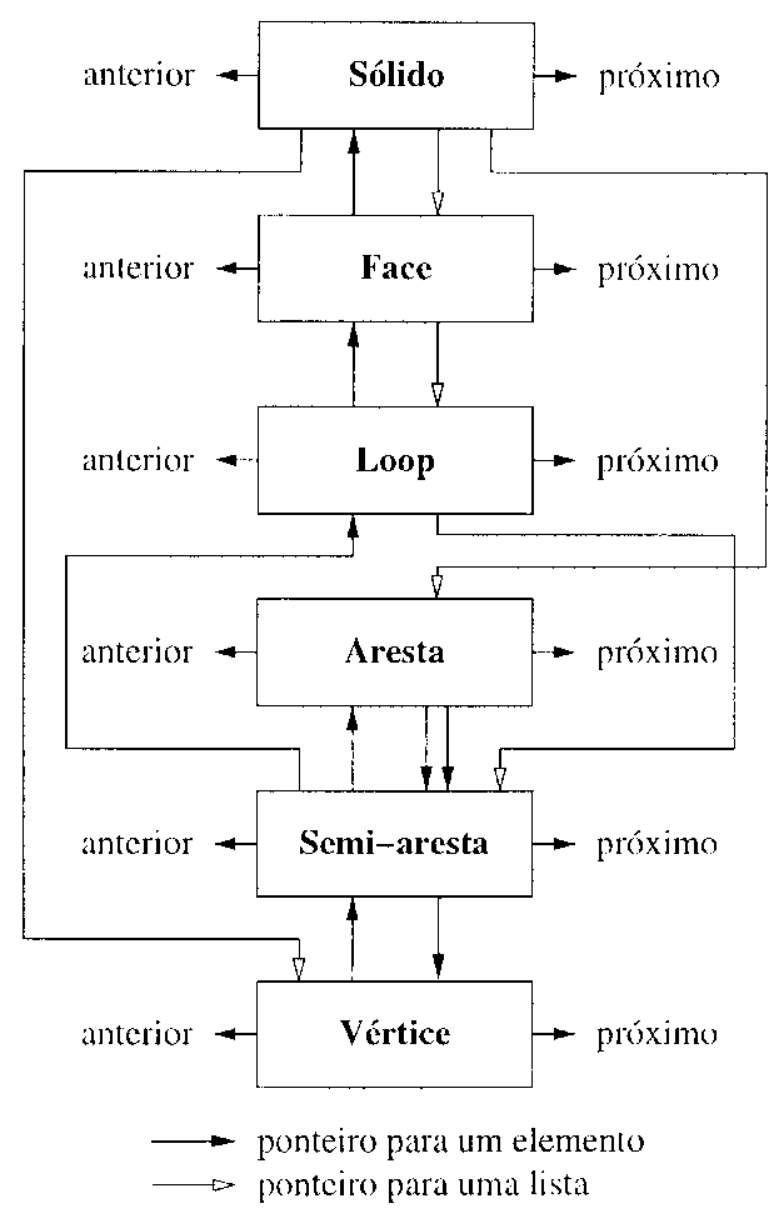

Figura 4.1: Níveis hicrárquicos da estrutura de dados "half-edge". 


\subsection{O Ambiente de Simulação FreeFlow-3D para Es- coamentos Não-Isotérmicos}

O ambiente de simulação FreeFlow-3D para escoamentos não-isotérmicos foi construído a partir de modificações nos módulos do sistcma Freoflow-3D. Com as modificações realizadas, o novo sistema é capaz de simular escoamentos não-isotérmicos com a viscosidade variando em função da tempcratura ou com a viscosiclade constante dependendo da escolha do usúario. A seguir será apresentada as modificações nos módulos do sistema.

\subsubsection{O Modelador}

É o módulo responsável pela inicialização dos dados para a simulação do escoamento. Este módulo possui uma interface gráfica para a introdução dos darlos referentes ao escoamento, como a modelagem do domínio, recipientes, injetores e fluidos que fazem parte da simulação e também os dados que configuram o domínio da simulacão, como tamanho da malha, parâmetros de escala entre ontros.

Para escoamentos năo-isotérmicos além de especificar os dados do escoamento, o usuário deverá escolher se deseja fazer uma simulaģăo com ou sem a influência da tomperatura como mostra a figura 4.2 .

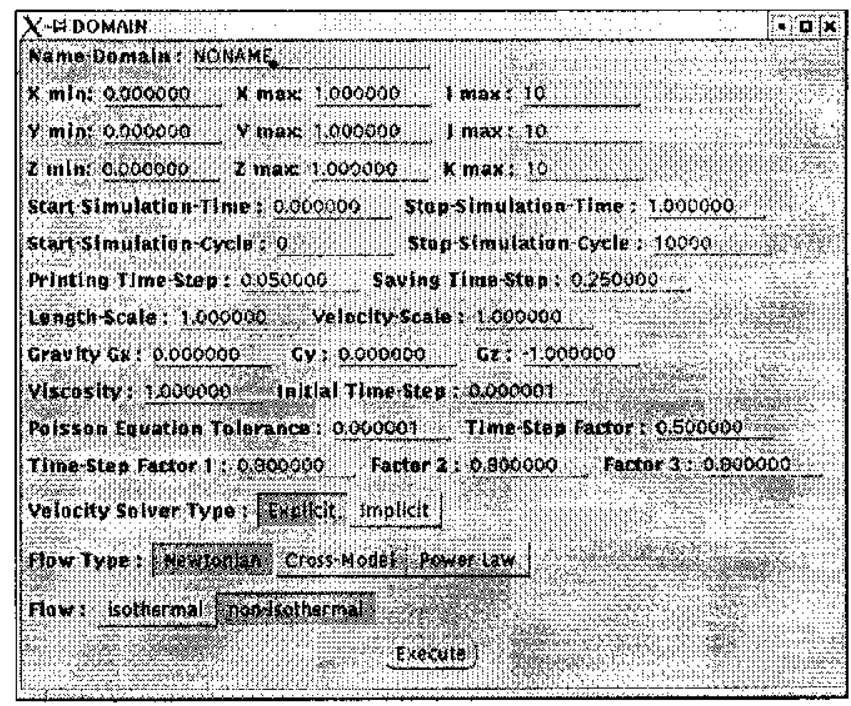

Figura 4.2: Entrada de dados.

Se a opção for por uma simulação com influência da temperatura, entäo os dados 
das propriedades térmicas do fluido terão de ser específicalos, além dla temperatura do ambiente e as temperaturas máxima e mínima utilizadas na simulação. Também pode-so escolher o modelo da viscosidade variando com a temperatura, isto é, entre um modelo para fluidos líquidos ou gasosos ou se a viscosidade não irá variar com a temperatura. So for escolhido $u$ modelo da viscosidade variando com a temperatura então as constantes utilizadas no respectivo modelo deverão ser especificadas, conforme secçào 1.4 .2 , como mostra a figura 4.3 .

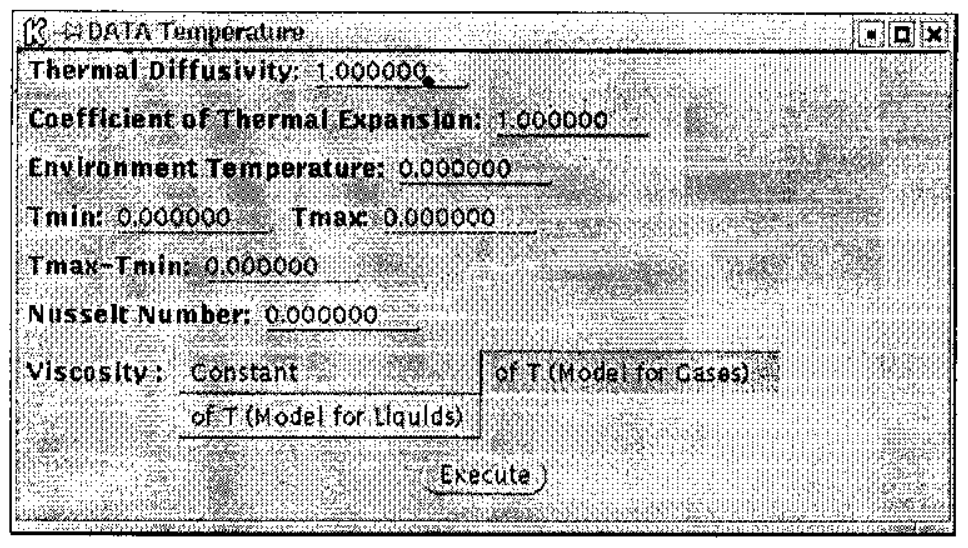

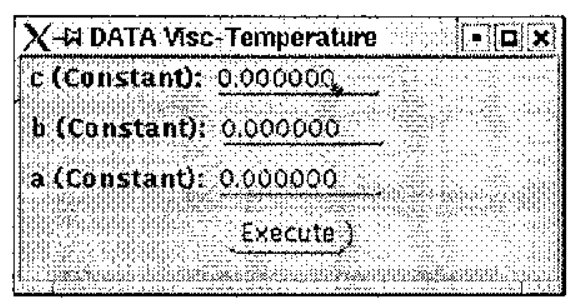

Figura 4.3: Entrada dados para simulação de escoamentos nãa-isotérmicos.

O próximo passo é a insersão de objetos geométricos que representem os recipientes, fluidos e/ou injetores envolvidos na simulação. No caso de uma simulação com a influência da temperatura quando for inserido um recipiente deverá ser especificada a temperatura do recipiente e também a condição de contorno para a temperatura que pode ser do tipo Dirichlet on adiabática. Se um injetor foi inserido, então deverá ser especificanda. a temperatura do fluido a ser injetado, e no caso da inserção de um fluido deverá scr especificada a temperatura do fluido. A figura 4.4 mostra a janela principal do módulo Modflow-3D para escoamentos não-isotérmicos com o modelo para a simulação de uma injeção de um líquido em uma caixa aberta e também a jancla de entrada dados para a inserção do recipiente do tipo "open box".

\subsubsection{O Simulador}

Este é o principal módulo do ambiente de simulação FreeFlow-3D para escoamentos não-isotérmicos. Nesset módulo são resolvidas as equagoós que modelam o escoamento de fluidos com influência da temperatıra. Este módulo năo possui uma interface gráfica e 

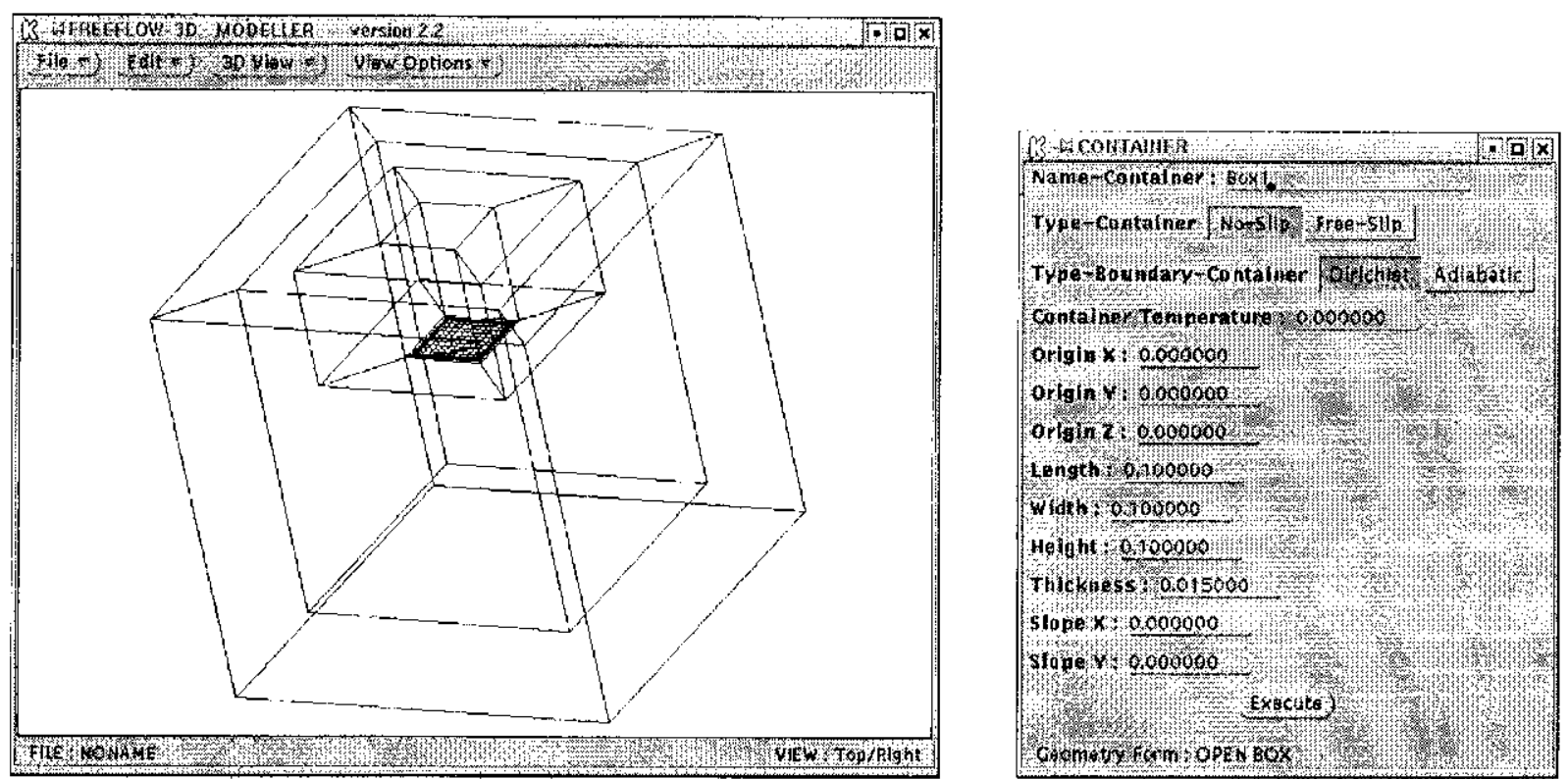

Figura 4.4: Interface do módulo Modflow-3D para escoamentos não-isotérmicos, com o modelo para a simulação de uma injeção de um líquido e a janela de cntrada dados do objeto do tipo "open box".

sua execução depende de uma sintaxe dada em linha de comando, o que permite que o programa seja executado em segundo plano. Para fazer uma simulação, o Simflow-3D executa os seguinte passos:

- leitura dos dados de entrada que são arquivos feitos pelo modelador;

- cálculo dos passos descritos no capítulo 2.

() módulo produz três tipos de saídas: uma normal em que a simulação poderá ou não continuar a partir destes dados, uma de segurança que pode ser usada para recuperar uma simulaçào interrompida antes de seu término e uma saída gráfica que pode ser interpretada pelo módulo visualizador.

\subsubsection{O Visualizador}

() Visflow-3D é o módulo responsável pela visualizaçăo dos dados gerados pelo simulador. Neste módulo estão implementadas técnicas de visualizargão de imagens tridimensionais, com recursos do iluminaçăo o transparencia. 
Pode-se visualizar os campos de velocidade em cada direção, pressão, temperatura, viscosidade através de uma escala de cores, de modo facilitar a interpretação de tais resultados. A figura 4.5 mostra a visualização de um escoamento não-isotérmico. A figura 4.6 mostra alguns recursos de visualização do módulo.
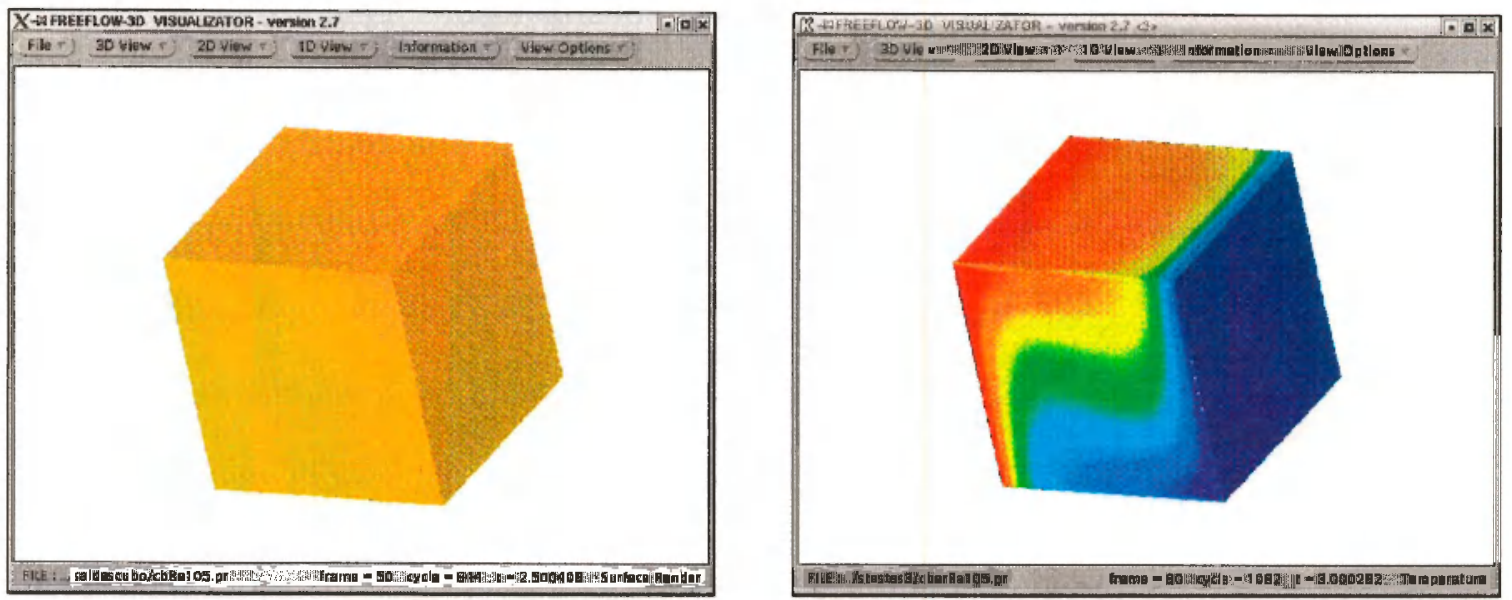

Figura 4.5: Visualização de um escoamento não-isotérmico: "rendering" tridimensional com recurso de iluminação (esquerda) e visualização da temperatura (direita).
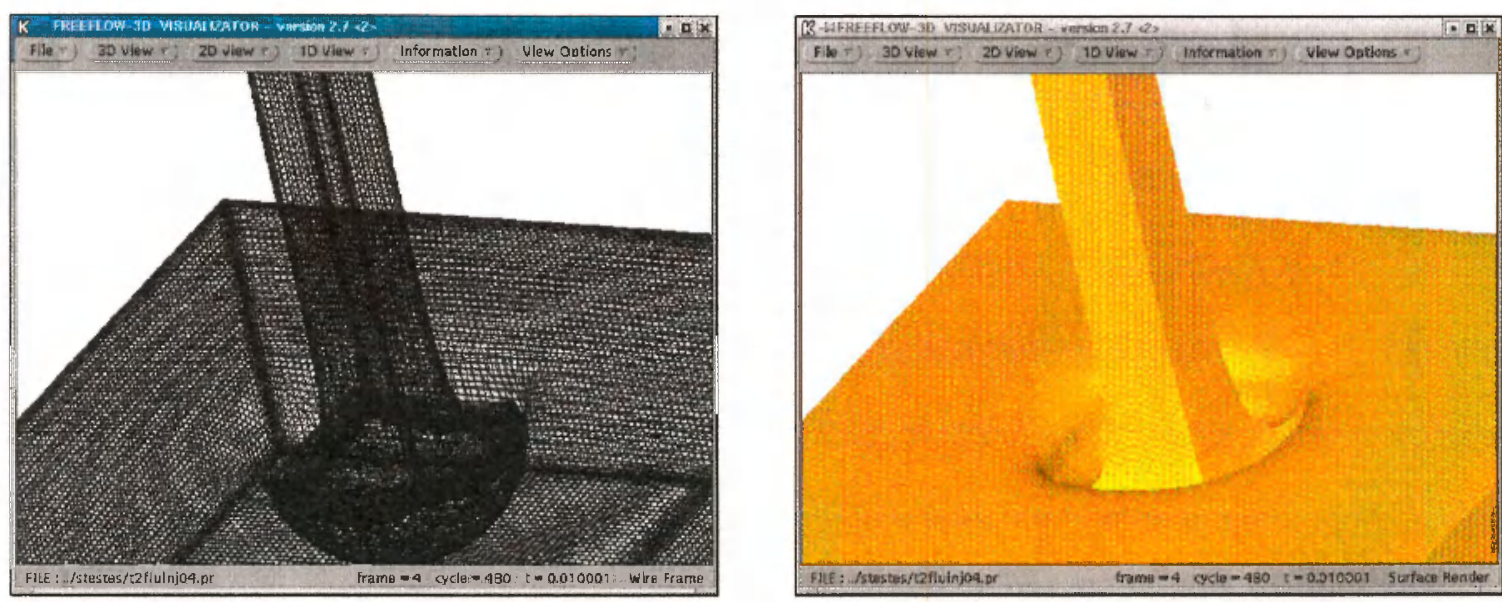

Figura 4.6: Alguns recursos de visualização do módulo Visflow-3D. 


\section{Capítulo 5}

\section{Resultados Numéricos}

Os resultados publicados na literatura sobre escoamentos não-isotérmicos são em modo geral de escoamentos confinados com paredes aquecidas (conveçăo natural) ${ }^{1}$, em que a aproximação de Boussinesq é utilizada. Os casos mais freqüentes săo os problemas de fluidos confinados por paredes, em que as paredes laterais são mantidas a uma temperatura constante enquanto que as outras estão termicamente isoladas.

Por essisa razão e pela dificuldade de se encontrar resultados na literatura com superfícies livres, na primeira secção desse capítulo comparam-se os resultados do modelo de Boussinesq com os resultados obtidos por (Trick et al., 2000) para o caso da conveçào natural no cubo fechado, com o objetivo de validar o simulador para escoamentos nãoisotérmicos. Logo após faz-se uma comparação do resultado obtido em 11 ma simulaçăo da convecção natural no cubo fechado utilizando-se a aproximação de Boussinesq com uma simulação da convecção natural no cubo fechado utilizando-se a aproximação de Boussinesq e a viscosidade variando com a temperatura. Por fim apresentam-se algumas simulações de escoamentos não-isotérmicos.

\subsection{Conveç̧ão Natural no Cubo Fechado}

Esse problema, freqüentemente citado na literatura, consiste em um cubo fechado com a temperatura mantida constante nas faces esquerda e direita, o termicamente isoladas

\footnotetext{
${ }^{1}$ Quando o campo de velocidades é totalmente determinado pela temperatura, dá-se o nome de convecção natural (Pontes, 1999).
} 
nas outras faces, ver figura 5.1 .

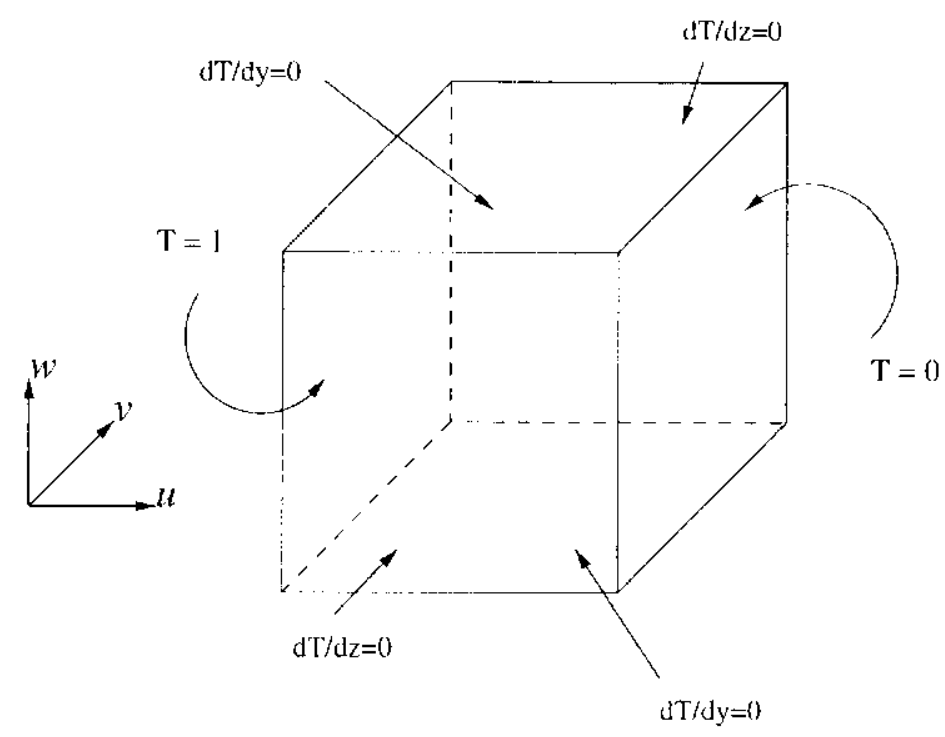

Figura 5.1: Modelo do problema da conveção natural.

Os dados para esta simulação são: difusăo tórmica $\alpha=2.08 \times 10^{-5} \frac{\mathrm{m} n^{2}}{\mathrm{~s}}$; coeficiente de expansão térmica $\beta=3.40 \times 10^{-3} K^{-1}$; viscosidade cinemática $\nu=1.5 \times 10^{-51} \frac{m^{2}}{\mathrm{~s}}$; temperatura inicial do fluido $293 \mathrm{~K}$; parannetros adimensionais $\mathrm{Re}=1.4$, Pr $=0.72$. Para

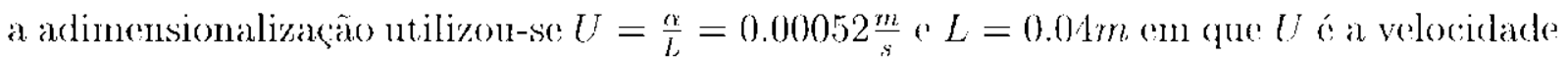
de refêrencia e $L$ o comprimento de referencia. Esse problema foi simulado em uma malha de $26 \times 26 \times 26$ células.

A tabela 5.1 compara os resultados mumćricos obtidos pela simulação do modelo de Boussinesq com os resultados obtidos por (Trick et al., 2000). Como pode se observar, pela tabcla 5.1 a análise dos resultados mostram pequenas diferenças em relação aos resultados obtidos por (Trick et. al., 2000). Para os números de Rayleigh $10^{3}, 10^{4}$ e $10^{5}$ apresentam-se figuras ilustrativas da temperatura (ver figura 5.2), linhas de temperatura (ver figura 5.3) e curvas de niveis das volocidades (ver figuras 5.4 e 5.5) ambas para o plano $x z$ médio, as quais se comparam satisfatoriamente com as figuras encontradas na literatura, como por exemplo em (Silva, 2002; De Vahl, 1983).

A seguir é feita uma comparação do resultado mostrado anteriomente na simulação da conveç̧ão natural no cubo fechado com uma simulação da conveção natural no cubo fechado utilizando-se a aproximação de Boussinesq e a viscosidade variando om função da temperatura, para isso utiliza-so a mesma modelagem da figura 5.1. A figura 5.6 mostra 


\begin{tabular}{||c||c|c||r|r||r|r||}
\hline \hline \multicolumn{1}{||c||}{} & \multicolumn{2}{c||}{$\mathrm{Ra}=10^{3}$} & \multicolumn{2}{c||}{$\mathrm{Ra}=10^{4}$} & \multicolumn{2}{c||}{$\mathrm{Ra}=10^{5}$} \\
\cline { 2 - 7 } & $\mathrm{SO}$ & \multicolumn{1}{c||}{ Ref. } & \multicolumn{1}{c||}{$\mathrm{SO}$} & \multicolumn{1}{c|}{ Ref. } & \multicolumn{1}{c|}{$\mathrm{SO}$} & \multicolumn{1}{c|}{ Ref. } \\
\hline \hline$u_{\text {max }}$ & 3.521 & 3.543 & 17.327 & 16.719 & 45.908 & 43.900 \\
$v_{\max }$ & 0.143 & 0.173 & 2.348 & 2.156 & 8.477 & 9.690 \\
$w_{\text {max }}$ & 3.630 & 3.544 & 20.040 & 18.983 & 70.396 & 71.060 \\
\hline \hline
\end{tabular}

Tabela 5.1: Comparação das velocidades máximas para os números Rayleigh $10^{3}, 10^{4} \mathrm{e}$ 105 . SO: Solução Obtida. Ref.: Referência (Trick et al., 2000).

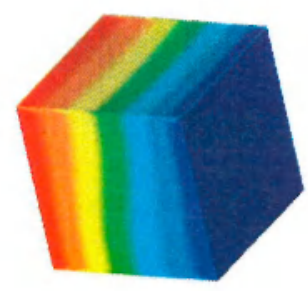

(a)

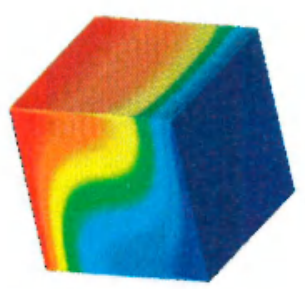

(b)

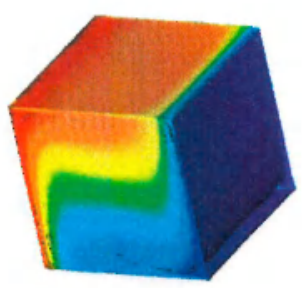

(c)

Figura 5.2: Visualização da temperatura. (a) $\mathrm{Ra}=10^{3}$; (b) $\mathrm{Ra}=10^{4}$; (c) $\mathrm{Ra}=10^{5}$.

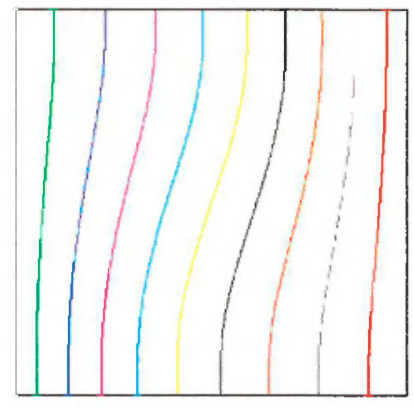

(a)

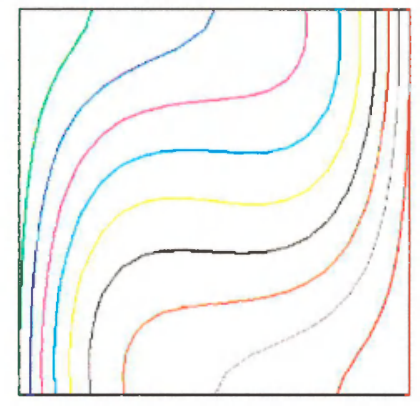

(b)

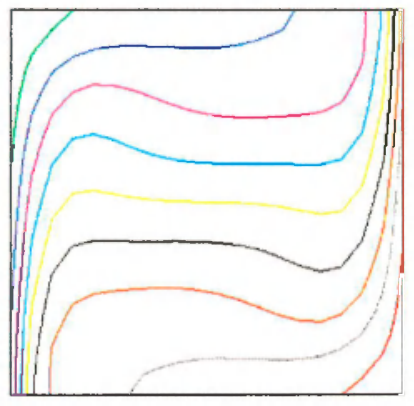

(c)

Figura 5.3: Linhas de temperatura. (a) $\mathrm{Ra}=10^{3}$; (b) $\mathrm{Ra}=10^{4}$; (c) $\mathrm{Ra}=10^{5}$.

o gráfico de um conjunto de dados conhecidos da viscosidade em função da temperatura e a curva que ajusta esses dados, para o cálculo da viscosidade utilizou-se o modelo (1.18) apresentado no capítulo 1 . Pode se observar pela tabela 5.2 que com o crescimento da viscosidade em função da temperatura (ver figura 5.6) as velocidades máximas da simulação utilizando o modelo de Boussinesq com a viscosidade variando em função da temperatura 


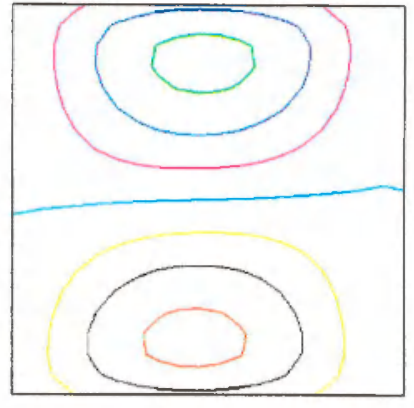

(a)

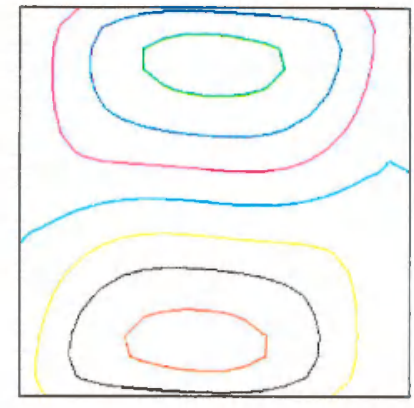

(b)

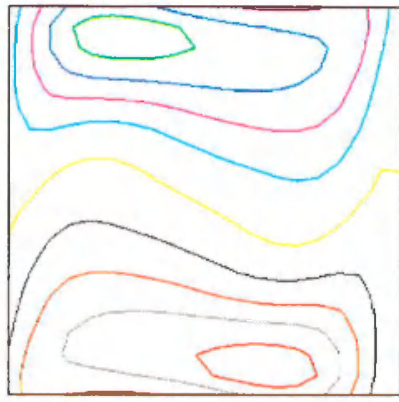

(c)

Figura 5.4: Curvas de níveis da velocidade $u$. (a) $\mathrm{Ra}=10^{3}$; (b) $\mathrm{Ra}=10^{4}$; (c) $\mathrm{Ra}=10^{5}$.

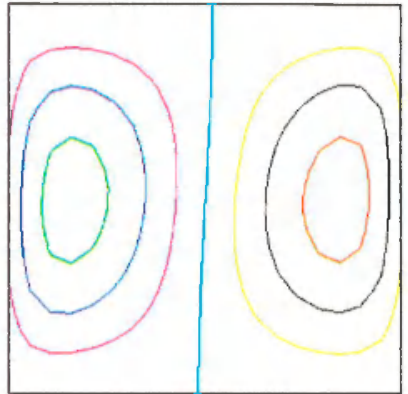

(a)

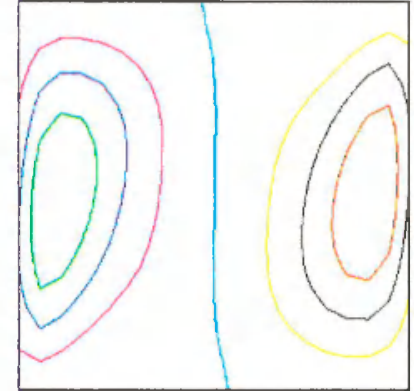

(b)

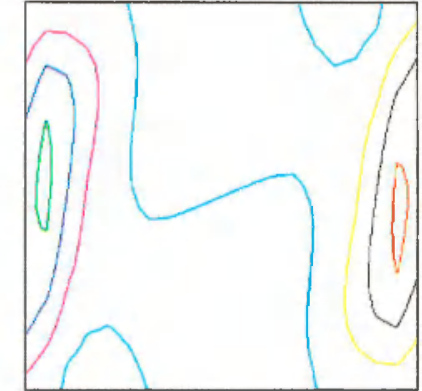

(c)

Figura 5.5: Curvas de níveis da velocidade $z$. (a) $\mathrm{Ra}=10^{3}$; (b) $\mathrm{Ra}=10^{4}$; (c) $\mathrm{Ra}=10^{5}$.

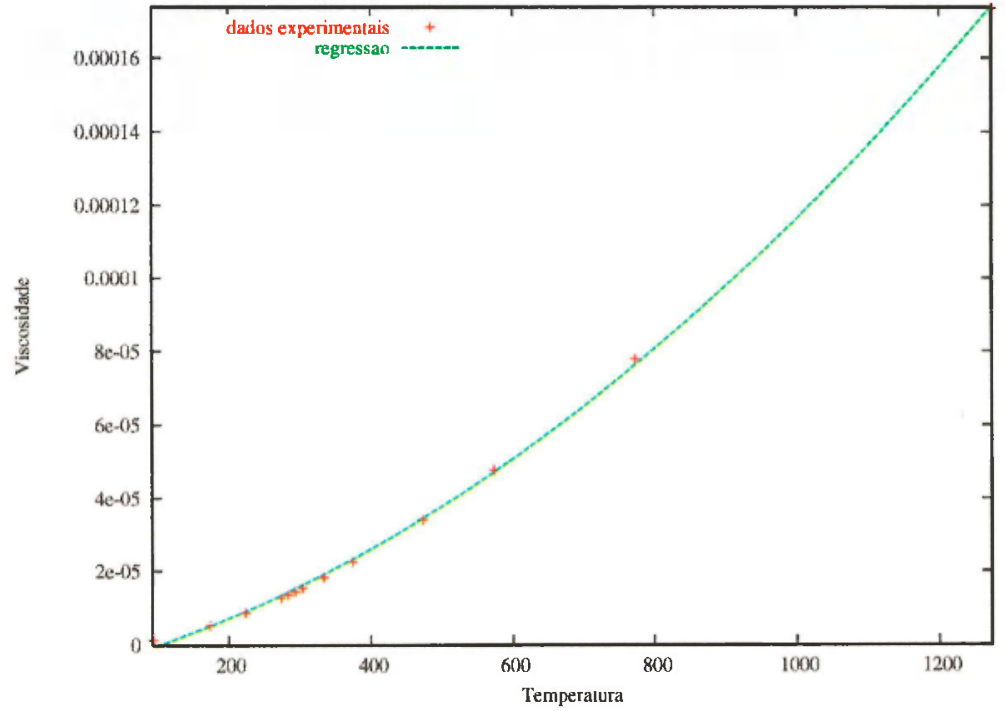

Figura 5.6: Ajuste de um conjunto de dados pelo método dos mínimos quadrados utilizando-se o modelo (1.18) para o cálculo da viscosidade. 
ficaram menores do que as velocidades máximas obtidas na simulação utilizando o modelo de Boussinesq com a viscosidade mantida constante. A figura 5.7 mostra as linhas de temperatura para o modelo de Boussinesq onde a viscosidade é constante e também onde a viscosidade varia com a temperatura.

\begin{tabular}{||l||r|r||}
\hline \hline & \multicolumn{1}{|c||}{$(\mathrm{a})$} & \multicolumn{1}{c|}{$(\mathrm{b})$} \\
\hline \hline$u_{\max }$ & 17.327 & 13.633 \\
$v_{\max }$ & 2.348 & 1.132 \\
$w_{\max }$ & 20.040 & 14.442 \\
\hline \hline
\end{tabular}

Tabela 5.2: Comparação das velocidades máximas para o número Rayleigh $10^{4}$. (a) Modelo de Boussinesq com a viscosidade constante; (b) Modelo de Boussinesq com a viscosidade variando em função da temperatura.

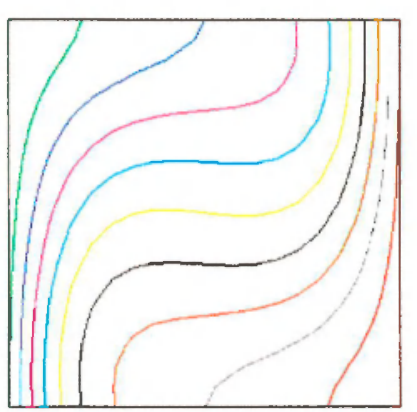

(a)

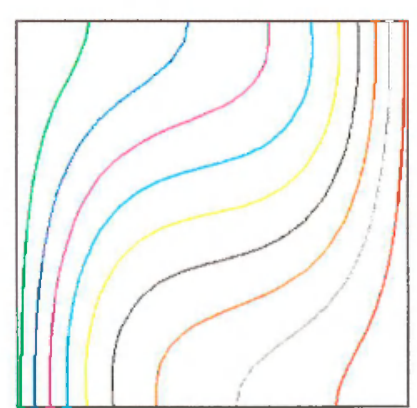

(b)

Figura 5.7: Linhas de temperatura, $\mathrm{Ra}=10^{4}$. (a) Modelo de Boussinesq com a viscosidade constante; (b) Modelo de Boussinesq com a viscosidade variando em função da temperatura.

Para se ter evidências de que o método numérico é convergente com o refinamento da malha foram realizadas simulações do problema da convecção natural, para os números de Rayleigh $10^{3}, 10^{4}$ e $10^{5}$, em três malhas diferentes: $10 \times 10 \times 10,20 \times 20 \times 20$ e $40 \times 40 \times 40$ células de fluido. As tabelas 5.3, 5.4 e 5.5 mostram os resultados obtidos.

Com respeito à estimativa da taxa de convergência, foram realizadas simulações do problema da convecção natural para o número de Rayleigh $10^{4} \mathrm{em}$ malhás com espaçamentos diferentes. Como não se conhece a solução analítica deste problema, utilizamse os dados da malha mais fina como solução analítica e calcula-se na norma $\|\cdot\|_{\infty}$ os erros 


\begin{tabular}{|c|ccc|}
\hline Mallia & $10^{3}$ & $20^{3}$ & $40^{3}$ \\
\hline \hline$u_{\text {max }}$ & 3.870 & 3.633 & 3.536 \\
$v_{\text {max }}$ & 0.163 & 0.162 & 0.158 \\
$w_{\text {max }}$ & 3.915 & 3.737 & 3.599 \\
\hline
\end{tabular}

Tabela 5.3: Comparação das velocidades máximas para o número de Rayleigh $10^{3}$.

\begin{tabular}{|c|ccc|}
\hline Malha & $10^{3}$ & $20^{3}$ & $40^{3}$ \\
\hline \hline$u_{\max }$ & 17.295 & 16.735 & 16.800 \\
$v_{\max }$ & 1.830 & 2.175 & 2.221 \\
$w_{\max }$ & 18.243 & 19.537 & 19.438 \\
\hline
\end{tabular}

Tabela 5.4: Comparação das velocidades máximas para o número de Rayleigh $10^{4}$.

\begin{tabular}{|c|ccc|}
\hline Malhat & $10^{3}$ & $20^{3}$ & $40^{3}$ \\
\hline \hline$u_{\text {max }}$ & 59.519 & 46.242 & 44.388 \\
$v_{\text {max }}$ & 7.136 & 8.467 & 9.166 \\
$w_{\text {max }}$ & 77.370 & 70.276 & 71.321 \\
\hline
\end{tabular}

Tabcla 5.5: Comparação das velocidades máximas para o número de Rayleigh 10".

entre essa malha e as outras, para as componentes da velocidade. Os espaçamentos utilizados nas simulações com as 5 malhas foram: malha $1(\delta=0.002)$, malha $2(\delta=0.002 j)$, malha $3(\delta=0.00133)$, malha $4(\delta=0.001)$ e malha $5(\delta=0.00066)$. Para se obter a estimativa da taxa de convergencia, fez-se um ajuste por mínimos quadrados vtilizando os dados obtidos juntamente com o espaçamento das malhas. A taxa de convergência é então estimada pela inclinação da linha ajustada. A figura 5.8 mostra a convêrgencia do método. A inclinaçäo para as normas da componente da velocidade ú 1.3 e para as normas da componente da velocidade $w$ é 2.1 .

Assim, os resultados obtidos indicam que o método apresentado converge com ordem ao menos 1. 


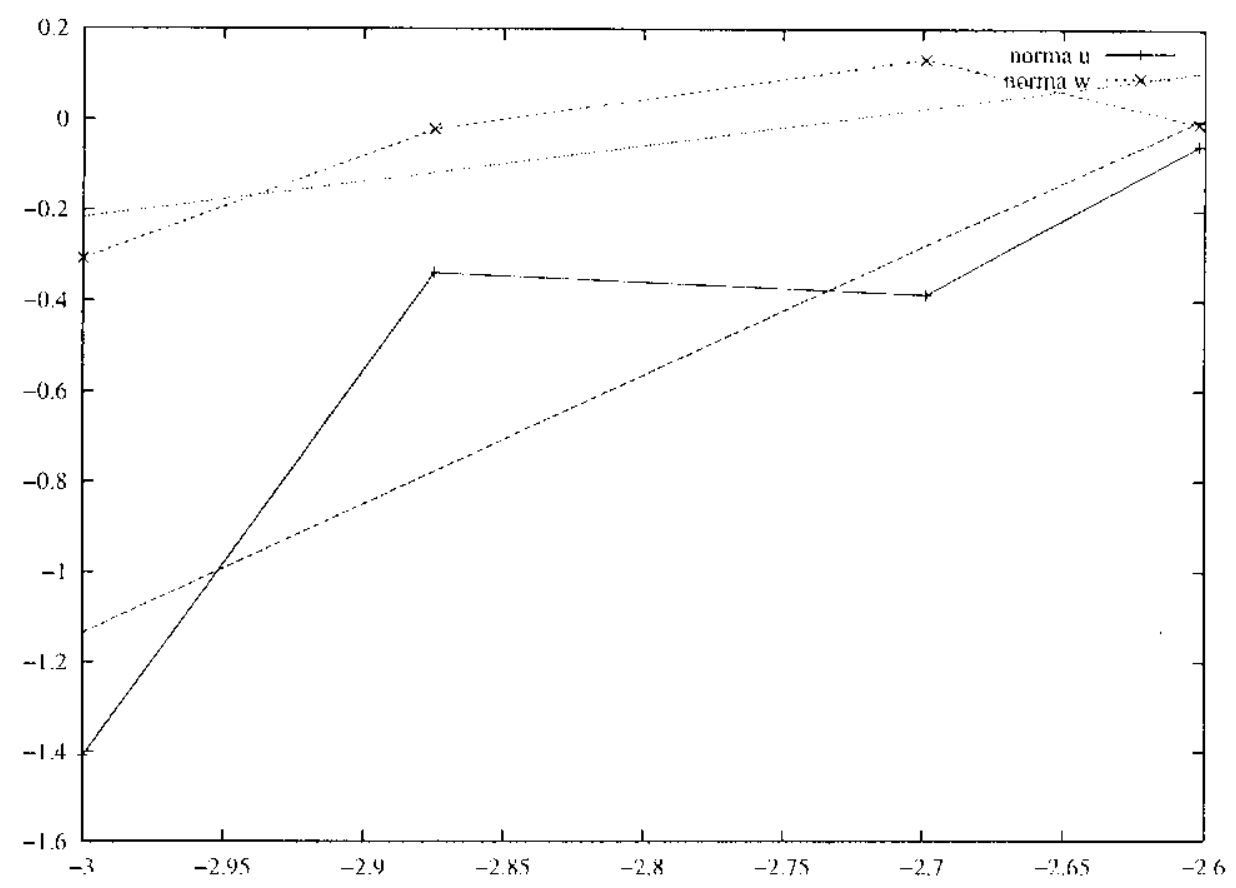

Figura 5.8: Normas $\|\cdot\|_{\infty}$ dos erros das componentes de velocidade para cada malha, em escalia logarítimica.

\subsection{Outros Exemplos Numéricos}

Nessa seçào são apresentados alguns exemplos de escoamentos näo-isotérmicos.

\section{Exemplo 1}

Essa simulaçăo consiste em uma caixa retangular fechada com razão 2:1:1 (comprimento:largura:altura) em que a temperatura é mantida constiante nas laces inferior e stperior a termicamente isolada nas outras faces, diferentemente das simulaçoon anteriores no cubo fechado em que se tinha as faces esquerda e direita mantidas a uma temperatura constante e as outras faces termicamente isoladas. A figura final dessa simulação (ver figura 5.9) se? compara satisfatoriamente com a figura encontrada em (Silva, 2002). Os dados utilizados para essa simulação extraídos de (Silva, 2002) são: difusão térmica $\alpha=2.08 \times 10^{-5} \frac{\mathrm{m}^{2}}{\mathrm{~s}}$; coeficiente de expansão térmica $\beta=3.40 \times 10^{-3} \mathrm{~K}^{-1}$ : viscosidade cinemática $\nu=1.5 \times 10^{-5} \frac{\mathrm{m}^{2}}{\mathrm{~s}}$; temperatura inicial do fluido $293 \mathrm{~K} ; \mathrm{Re}=1.4 ; \mathrm{Pr}=0.7^{2} ; \mathrm{Ra}=3 \times 10^{4} ;$ escala de comprimento $L=0.04 m$; velocidade de referência $U=\frac{\alpha}{l}=0.00052 \frac{m}{s}$. Esse problema loi simulado em uma malha de $26 \times 16 \times 16$ células. 


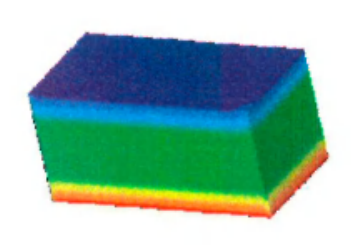

$+0.0000+0.143+0.2000+0.429+0.971+0.714+0.857+2.000$
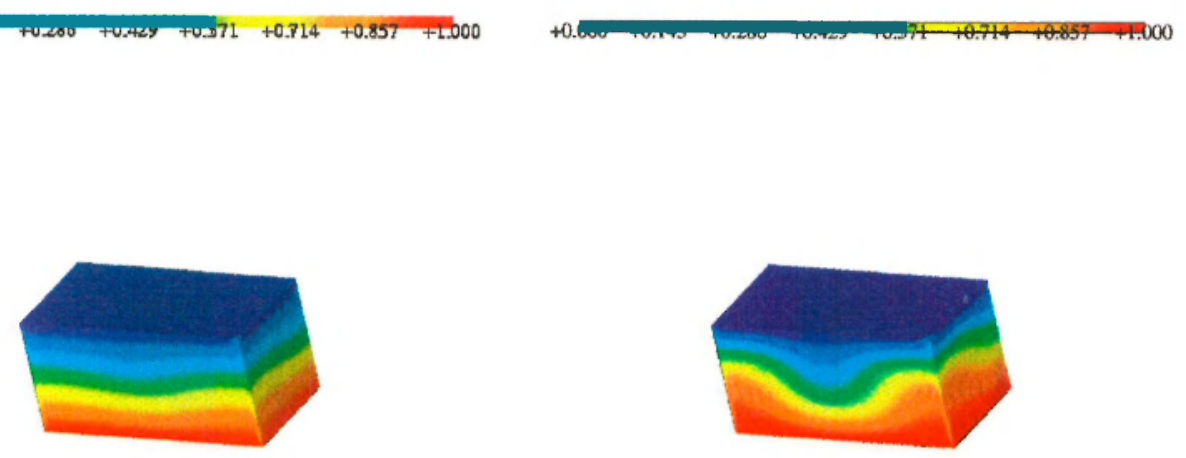

Figura 5.9: Visualização da temperatura em um recipiente com razão 2:1:1.

\section{Exemplo 2}

O objetivo dessa simulação é mostrar um fluido sendo injetado a uma certa temperatura no mesmo fluido contido dentro de um recipiente com uma temperatura menor e verificar o comportamento do fluido quando ele entrar em contato com o fluido no recipiente, ver figura 5.10 .

A temperatura do ambiente é a mesma do fluido sendo injetado e a temperatura do recipiente é a mesma do fluido contido no recipiente. Nesse exemplo utilizou-se um número de Nusselt relativamente alto para observar-se melhor as trocas de calor entre o ambiente e o fluido contido no recipiente. Para essa simulação utilizaram-se os seguintes dados: dimensão do domínio $0.01 \times 0.01 \times 0.01 \mathrm{~m}$; escala de velocidade $U=1.0 \frac{\mathrm{m}}{\mathrm{s}}$; escala de comprimento $L=1 \times 10^{-3} \mathrm{~m}$; viscosidade cinemática $\nu=1 \times 10^{-4} \frac{\mathrm{m}^{2}}{\mathrm{~s}}$; difusão térmica $\alpha=2 \times 10^{-5} \frac{\mathrm{m}^{2}}{\mathrm{~s}} ; \mathrm{Re}=10 ; \mathrm{Pr}=5 ; \mathrm{Nu}=1 \times 10^{3} ;$ temperatura inicial do fluido $293 \mathrm{~K} ;$ temperatura do ambiente $293 K$; temperatura do recipiente $243 K$; temperatura do fluido 


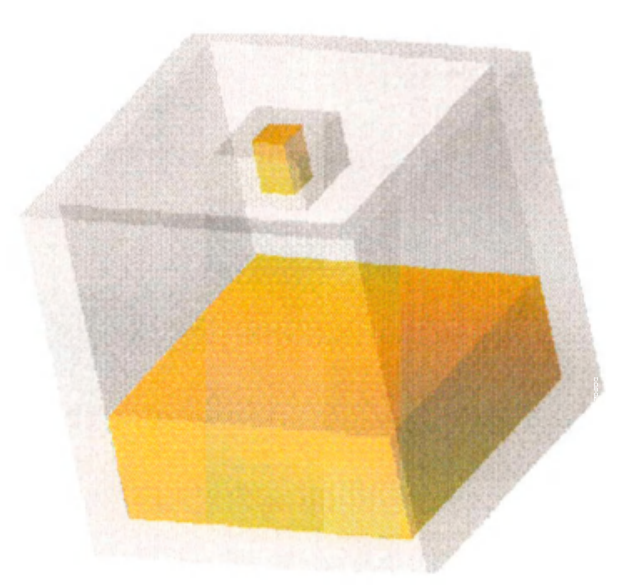

Figura 5.10: Visualização do modelo.

contido no recipiente $243 K$. Esse problema foi simulado em uma malha de $40 \times 40 \times 40$ células. Pelas figuras 5.11 e 5.12 pode se observar que o fluido injetado está sendo resfriado pelo fluido que está contido no recipiente. Esse exemplo ilustra a capacidade do sistema FreeFlow-3D para escoamentos não-isotérmicos em lidar com diferentes temperaturas simultaneamente.

\section{Exemplo 3}

Esse exemplo apresenta a injeção de um fluido em um recipiente em que a temperatura no fundo do recipiente foi fixada pelo parabolóide representado na figura 5.13 dado pela expressão

$$
T(x, y)=T_{R} \frac{\left(x-x_{0}\right)\left(x-x_{2}\right)}{\left(x_{1}-x_{0}\right)\left(x_{1}-x_{2}\right)} \frac{\left(y-y_{0}\right)\left(y-y_{2}\right)}{\left(y_{1}-y_{0}\right)\left(y_{1}-y_{2}\right)}
$$

em que $x_{i}$ e $y_{i}, i=0,1,2$ são definidos como na figura 5.14 e $T_{R}$ é a temperatura no recipiente, as paredes laterais do recipiente estão termicamente isoladas.

Para essa simulação utilizaram-se os seguintes dados: dimensão do domínio $0.052 \times 0.052 \times$ $0.052 \mathrm{~m}$; escala de velocidade $U=1.0 \frac{\mathrm{m}}{\mathrm{s}}$; escala de comprimento $L=4 \times 10^{-2} \mathrm{~m}$; viscosidade cinemática $\nu=4 \times 10^{-3} \frac{\mathrm{m}^{2}}{\mathrm{~s}} ;$ difusão térmica $\alpha=2 \times 10^{-5} \frac{\mathrm{m}^{2}}{\mathrm{~s}} ; \operatorname{Re}=10 ; \operatorname{Pr}=200$; temperatura inicial do fluido $243 K$; temperatura do ambiente $243 K$; temperatura do recipiente $293 K$. Esse problema foi simulado em uma malha de $26 \times 26 \times 26$ células do fluido. Pelas figuras 5.15 e 5.16 pode se observar claramente o fluido sendo aquecido pelo recipiente segundo o perfil parabólico imposto na parede do fundo. Esse exemplo ilustra a capacidade do sistema FreeFlow-3D para escoamentos não-isotérmicos em lidar com 

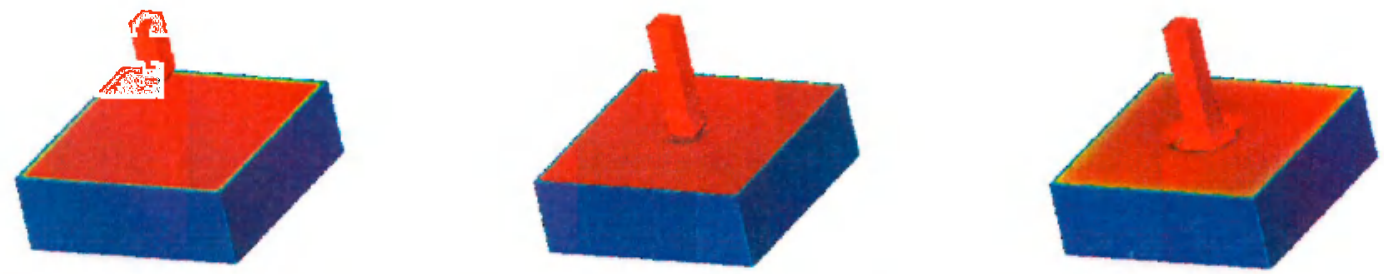

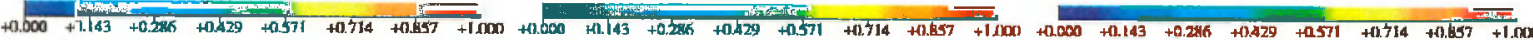

(a) $t=0.0025 \mathrm{~s}$

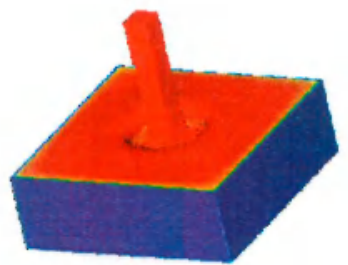

(b) $t=0.005 s$

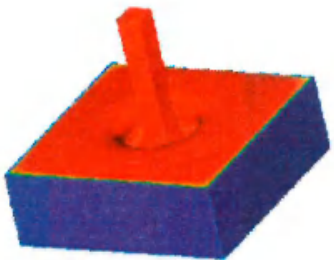

(c) $t=0.0075 \mathrm{~s}$

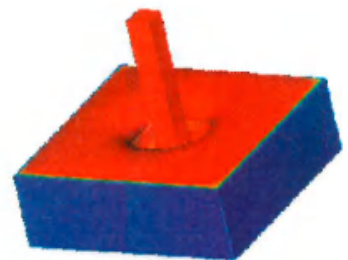

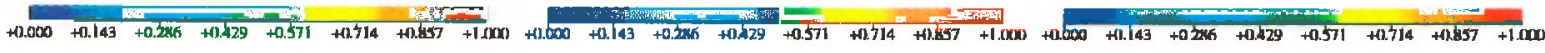

(d) $t=0.01 \mathrm{~s}$

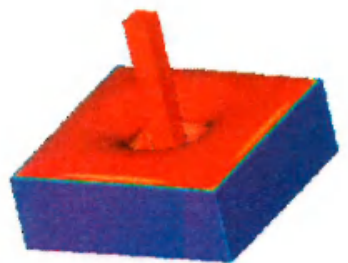

(e) $t=0.0125 \mathrm{~s}$

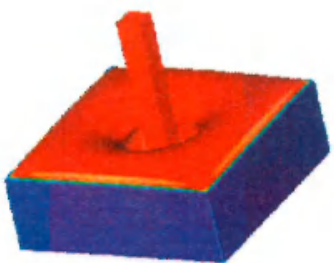

(f) $t=0.015 \mathrm{~s}$

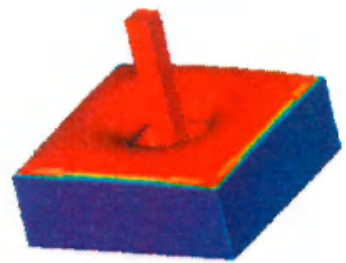

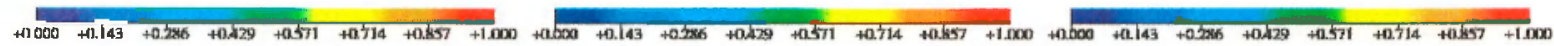

(g) $t=0.0175 \mathrm{~s}$

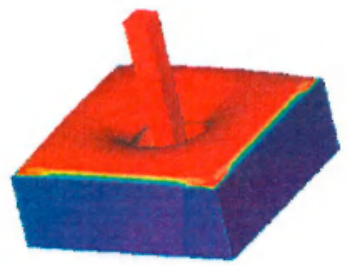

(h) $t=0.02 \mathrm{~s}$

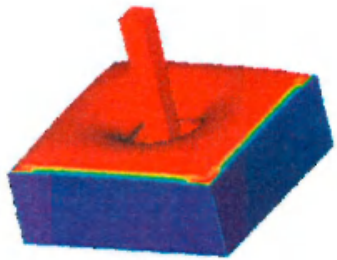

(i) $t=0.0225 \mathrm{~s}$

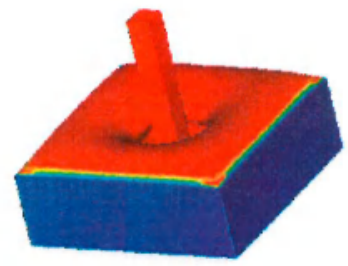

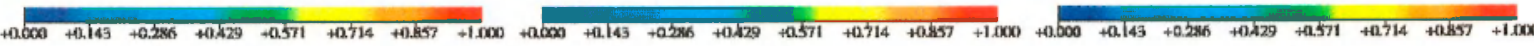

(j) $t=0.025 \mathrm{~s}$

(k) $t=0.0275 \mathrm{~s}$

(1) $t=0.03 \mathrm{~s}$

Figura 5.11: Visualização tridimensional da temperatura. 


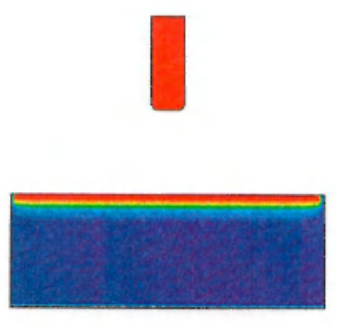

(a) $t=0.0025 \mathrm{~s}$

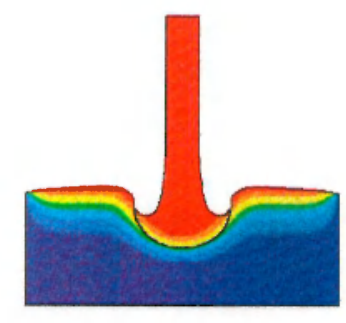

(d) $t=0.01 \mathrm{~s}$

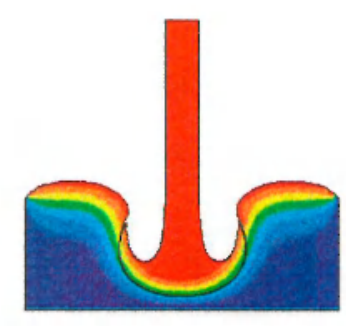

(g) $t=0.0175 \mathrm{~s}$

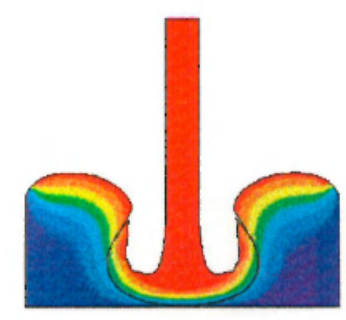

(j) $t=0.025 \mathrm{~s}$

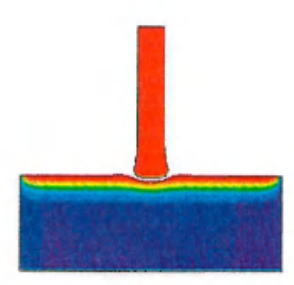

(b) $t=0.005 \mathrm{~s}$

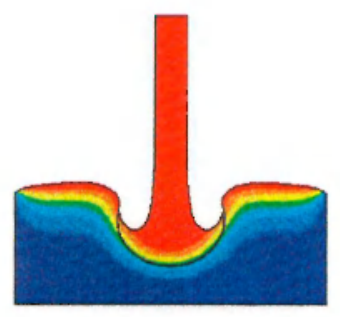

(e) $t=0.0125 \mathrm{~s}$

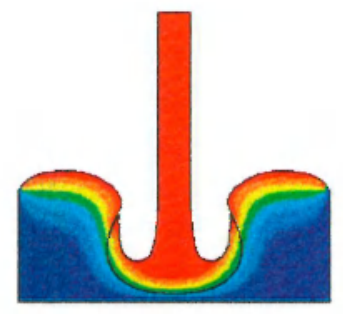

(h) $t=0.02 \mathrm{~s}$

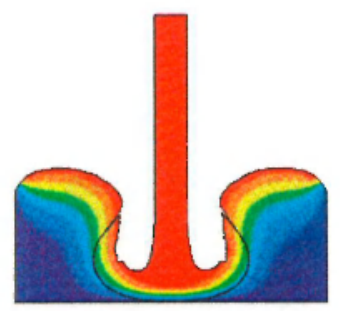

(k) $t=0.0275 \mathrm{~s}$

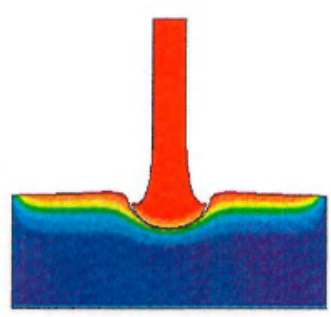

(c) $t=0.0075 \mathrm{~s}$

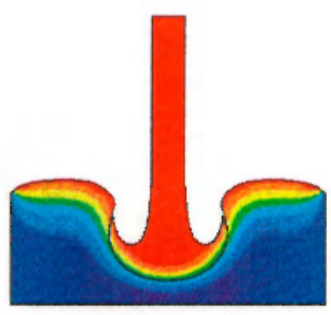

(f) $t=0.015 \mathrm{~s}$

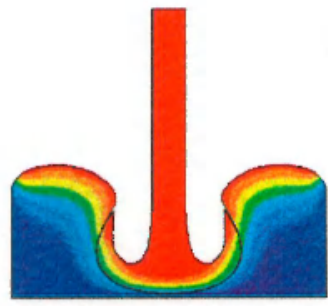

(i) $t=0.0225 \mathrm{~s}$

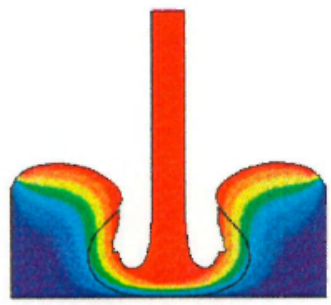

(l) $t=0.03 \mathrm{~s}$

Figura 5.12: Visualização bidimensional da temperatura no plano $x z$ referente a figura 5.11 . 


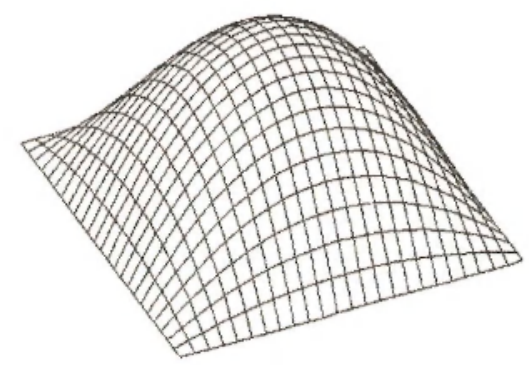

Figura 5.13: Representação do perfil de temperatura imposto no fundo do recipiente.

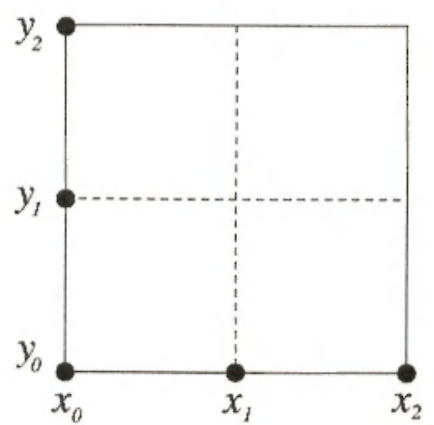

Figura 5.14: Representação do fundo do recipiente mostrando $x_{i}, y_{i}$, $i=0,1,2$.

diferentes condições de contorno e temperaturas simultaneamente.
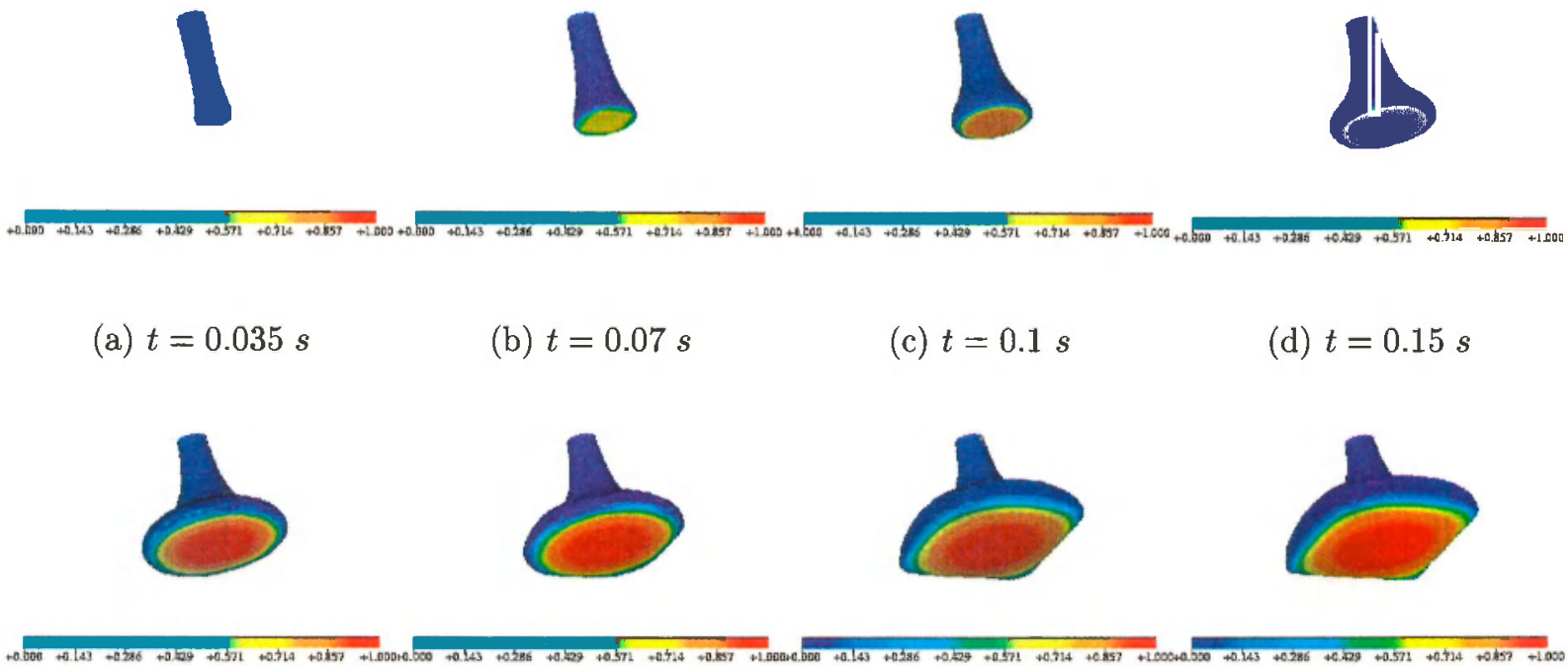

(e) $t=0.25 \mathrm{~s}$

(f) $t=0.3 \mathrm{~s}$

(g) $t=0.4 \mathrm{~s}$

(h) $t=0.45 \mathrm{~s}$
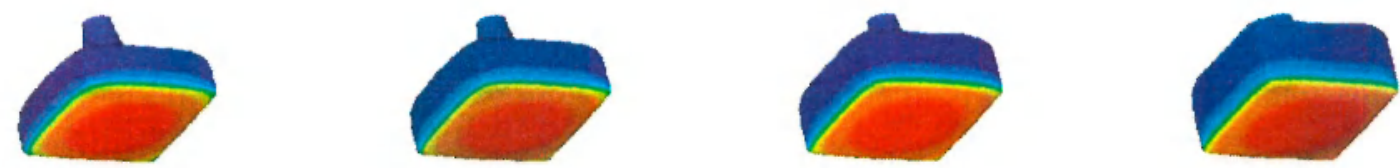
(i) $t=0.55 \mathrm{~s}$
(j) $t=0.6 \mathrm{~s}$
(k) $t=0.65 \mathrm{~s}$
(1) $t=0.75 s$

Figura 5.15: Visualização da temperatura. 

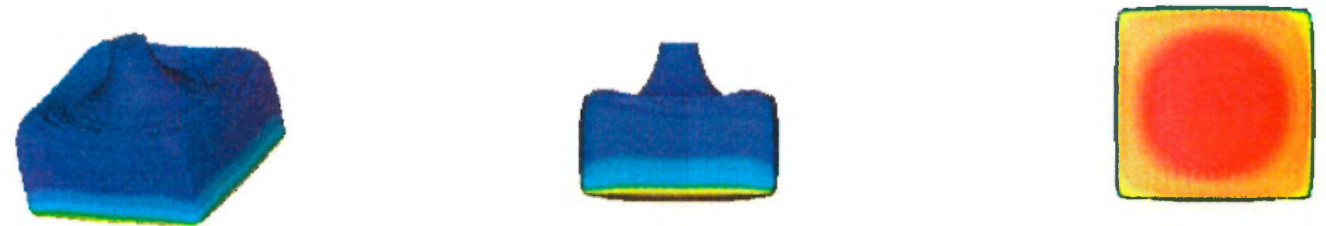

$0.000+0.143+0.286+0.429+0.571+0.114+0.857+1.000$

$+0.500+0.143+0.286+0.429+0.571+0.714+0.657+1.000+0.000+0.43+0.286+0.429+0.571+0.714+0.159+1.000$

Figura 5.16: Visualização da temperatura, $t=0.75 \mathrm{~s}$, por vários ângulos.

\section{Exemplo 4}

Esse exemplo apresenta a injeção em um recipiente em que a temperatura do recipiente é menor que a temperatura do fluido e tem por objetivo verificar o comportamento do fluido ao entrar em contato com o recipiente. Nesse exemplo a viscosidade varia em função da temperatura, sendo que a viscosidade do fluido cresce a medida que a temperatura decresce. A figura 5.17 mostra o conjunto de dados conhecidos da viscosidade em função da temperatura e a curva que ajusta esses dados, para o cálculo da viscosidade utilizou-se o modelo (1.19) apresentado no capítulo 1. Para essa simulação utilizaram-se os seguintes

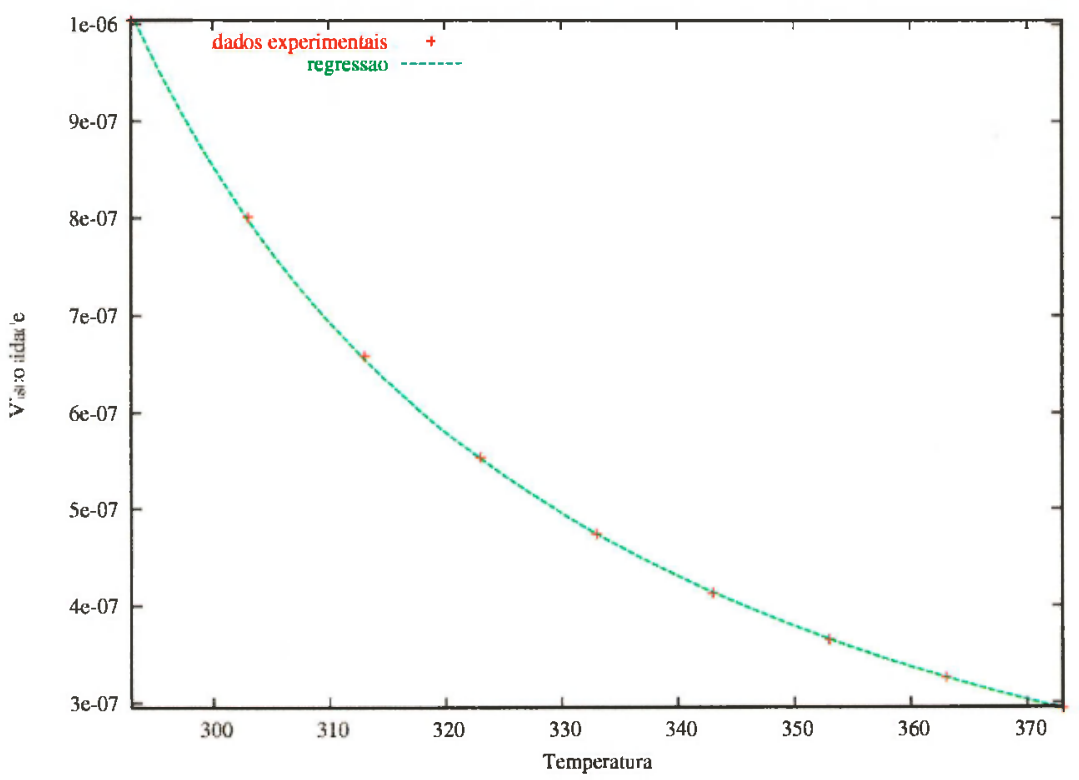

Figura 5.17: Ajuste de um conjunto de dados pelo método dos mínimos quadrados utilizando-se o modelo (1.19) para o cálculo da viscosidade.

dados: dimensão do domínio $0.001 \times 0.001 \times 0.001 \mathrm{~m}$; escala de velocidade $U=0.1 \frac{\mathrm{m}}{\mathrm{s}}$; escala de comprimento $L=1 \times 10^{-4} \mathrm{~m}$; viscosidade cinemática $\nu=10.04 \times 10^{-7} \frac{\mathrm{m}^{2}}{\mathrm{~s}}$; 
difusão térmica $\alpha=1.4 \times 10^{-7} \frac{m^{2}}{s} ; \operatorname{Re}=9.96 ; \operatorname{Pr}=7.07$; temperatura inicial do fluido $293 K$; temperatura do ambiente $293 K$ : temperatura do recipiente $273 K$. Esse problema foi simulado em uma malha de $20 \times 20 \times 20$ células. Pela figura 5.18 pode se observar o fluido sendo resfriado pelo recipiente, causando uma variação da viscosidade como pode ser visto na figura 5.19 .

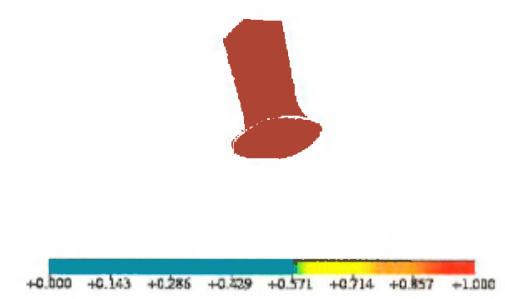

(a) $t=0.006 \mathrm{~s}$

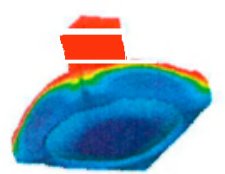

(d) $t=0.025 \mathrm{~s}$
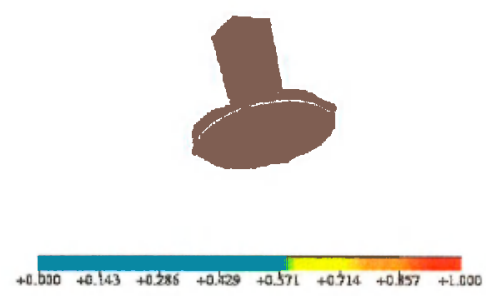

(b) $t=0.012 \mathrm{~s}$
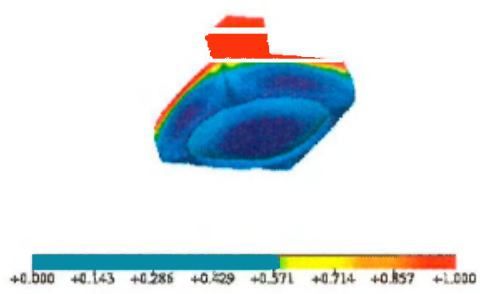

(e) $t=0.030 \mathrm{~s}$
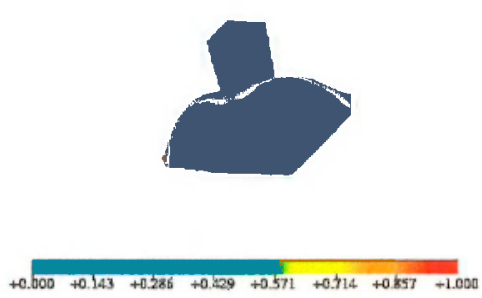

(c) $t=0.020 \mathrm{~s}$
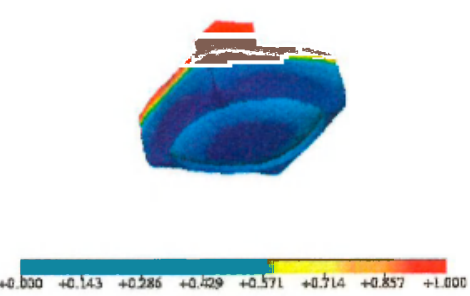

(f) $t=0.040 s$

Figura 5.18: Visualização da temperatura.

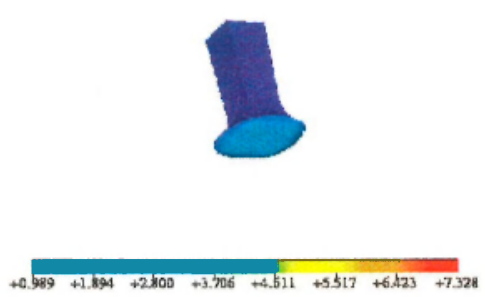

(a) $t=0.006 \mathrm{~s}$
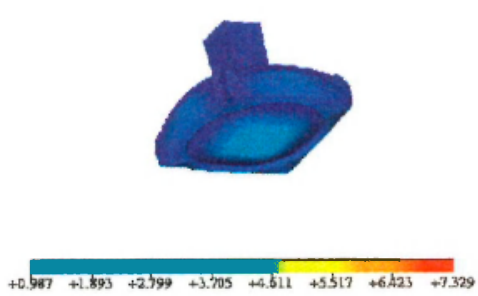

(b) $t=0.025 \mathrm{~s}$
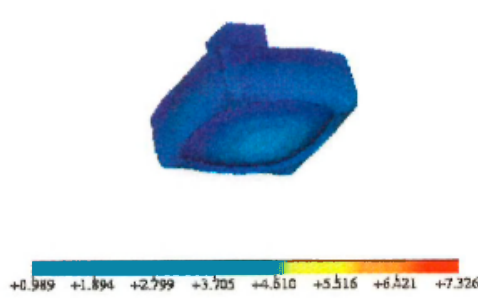

Figura 5.19: Visualização da viscosidade. 


\section{Capítulo 6}

\section{Conclusão}

Esse projeto teve como objetivo a simulação numérica de escoamentos com superfícies livres não-isotérmicos utilizando-se o modelo de Boussinesq e a viscosidade calculada como função da temperatura ou mantida constante dependendo da escolha do usuário do sistena. Isso implicou na extensão do sistema FreeFlow-3D, o qual anteriormente simulava escoamentos isotérmicos, assim no modelador de escoamentos tridimensionais foi feita modificaçoes permitindo a inserção das propriedades térmicas do fluido o a escolha das condiçoes de contorno apropriadas à temperatura nas fronteiras do escoamento; no simulador foi incorporada a equação da energia e feito o ajuste das equaçóes de NavierStokes para se poder simular escoamentos não-isotérmicos, ou seja, adaptoul-se o método GENSMAC-3D, ver capitulo 2, para esse tipo de problema o implementaram-se as condiçoes de fronteira apropriadas, ver capítulo 3 ; no visualizador acrescentou-se a opgão de visualização dos campos de temperatura c viscosidade.

No capítulo 5 , compararam-se os resultados obtidos pelo modelo de Boussinesq com os resultados obtidos por (Trick et al., 2000) para o caso cla conveção natural no cubo fechado usando o ar cono fluido para os números de Rayleigh $10^{3}, 10^{4}$ a $10^{5}$, pois esse tipo de simulação é muito citada na literatura. A solução numérica oblida apresenton resultados satisfatórios, indicando que o simulador pode produzir resultados numéricos satisfatórios em outras situaçós como pode ser visto nos outros exemplos apresentados. Portanto pode se concluir que o resultado deste trabalho atingiu o objetivo esperado, ou seja, permitindo que o sistema FreeFlow-3D seja capaz de modelar, simular e visualizar escoamentos não-isotérmicos. 
O sistcma FreeFlow-3D possui uma estrutura que permite sua extensão, assim como proposta de futuros trabalhos é a implementaçäo de outros tipos de escoamentos, como por exemplo a implementação de fluidos viscoelásticos com influência da temperatural. 


\section{Referências Bibliográficas}

Amsden, A. e Ilarlow, F. H. (1970). The SMAC Method: A Numerical Technique for Calculating Incompressible Fluid Flows. Los Alamos Scientific Lab. Report LA-4370.

Castelo Filho, A., Cuminato, J. A., Oliveira, M. C., Santi, R. D., Tomé, M. F., c César, C.

N. L. (1997). FreeFlow-3D: Um Sistema Integrado para Simulação de Escoamentos Incompressiveis com Superfícies Livres. Relatório 'Téenico, ICMC/USP.

Castelo Filho, A., Tomé, M. F., César, C. N. L., McIíe, S., e Cuminato, J. A. (1999).

FrecFlow: An Integrated Simulation System for Three-Dimensional Free Surface Hlows. Journal of Computing and Visualization in Science, 103:77--92.

De Vahl, D. G. (1983). A Natural Convection of Air in a Square Cavity: A Bench Mark Solution. Inlernational Journal of Numerical Methods in Fluids, 3:249 264.

Ferreira, V. G., Tomé, M. F., Mangiavacchi, N., Castelo, A., Cuminato, J. A., Fortuna, A. O., e McKee, S. (2002). High Order Upwinding and the Hydraulic Jump. International Journal of Numerical Methods in Fluids, 39:549-583.

Ferreira, V. L., Mello, O. D., Oliveira, J. N., c Fortuna, A. O. (1999). Tópicos Teóricos e Computacionais em Escoamentos de Fluidos. Notas do ICMC/USP.

Fletcher, C. A. J. (1988). Computational Techniques for Fluid Dynamics: Vol. I and Vol. II. Springer Verlag.

Fortuna, A. O. (2000). Técnicas Computacionais para Dinâmica dos Fluidos. Edusp.

Fox, R. W. e McDonald, A. T. (1995). Introduçäo à Dinâmica dos Fluidos. Livros Técnicos o Científicos. 
Gricbel, M., Thomas, D., e Nembeffer, T. (1997). Numerical Simulation in Fluid Dynamics. A Practical Introduction. Socicty for Inclustrial and Applied Mathematics.

Harlow, F. H. e Welch, J. E. (1965). Numerical Calculation of Time-Dependent Viscous Incompressible Flow of Fluid with Free Surface. The Physic of Fluids, 8(1):2182-2189.

Oliveira, J. (1999). Desenvolvimento de um Sistema de Simulação de Escoamentos de Fluidos com Superfícies Livres Bidimensionais. Dissertação de mestrado, ICMC/USP.

Paiva, L. S. (2000). Desenvolvimento de um Modelador de Movimentos para o Sistema FreeFlow-3D. Dissertação do mestrado, ICMC/LSP.

Peyret, R. c Taylor, T. D. (1983). Computational Methods for Fluid Flou. Springer-Verlag.

Pontes, J. (1999). Fenômenos de Transferência. Technical Report, COPPE/UFR.J.

Schmidt, F. W., Henderson, R. W., o Wolgemuth, C. H. (1996). Introduçăo à.s Cụ̂naizus Térmicas. Tormodinâmica, Mecânica dos Fluidos e Transferência de Calor. Edgard Blütcher Ltda.

Shewchuck, J. R. (1994). An Introduction to the Conjugate Gradient Method without. Agonizing Pain. School of Computer Science, Carnegie Mellon University.

Silva, J. M. (2002). Simulação Numérica de Escoamentos com Superfícies Livress e com Influência da Temperatura. Dissertação de mestrado, ICMC/USP.

Souza, F. S. (2002). Simulação Numérica de Fscoamentos Multifásicos usando o Sistema FrocFlow-3D. Dissertaçăo de mestrado, ICMC/USP.

Tomé, M. F., Castelo Filho, A., Cuminato, J. A., Mangiavacehi, N., e Mckee, S. (2001). GENSMAC3D: A Numerical Method for Solving Lnsteady Three-Dimensional Free Surface Flows. International Journal For Numerical Methods in Fluids, 37:747-796.

Tomé, M. F., Castelo Filho, A., Cuminato, J. A., e McKee, S. (1996a). GENSMAC-3D: Implementation of the Navier-Stokes Equation for 3D Free Surface Flows. Notas do ICMSC/USP. 
Tomé, M. F., Duffy, B., e Mckee, S. (1996b). A Numerical Methor for Solving Unsteady Non-Newtonian Free Surface Flows. Journal of Non-Newtonian Fluid Mechanies, $62: 934$.

Tomé, M. F. e Mckee, S. (1994). GENSMAC: A Computational Marker-and-Cell Method for Free Surface Flows in General Domains. Journal of Computational Physics, $110(1): 171-189$.

Trick, E., Labrosse, G., e Betrouni, M. (2000). A First Incursion into the 3I) Structure of Natural Convection of Air in a Differencially Heated Cubic Cavity. International Journal of Heat and Mass Transfer, 43:4043-4056. 